\title{
Predicting Future Monthly Electricity Consumption in the Philippines using Markov-Chain Grey Model
}

\author{
Jackie D. Urrutia ${ }^{1 *}$, Julius L. Meneses ${ }^{2}$ and Glenn Valerie A. Antonio ${ }^{3}$ \\ 'Research Management Office, Polytechnic University of the Philippines-Manila, \\ Philippines; jackieurrutia20@gmail.com \\ ${ }^{2}$ Programs, Research and Extension Services, Rizal Technological University, \\ Philippines; jlmeneses@rtu.edu.ph \\ ${ }^{3}$ Statistician I, Budget Division, L and Registration Authority, \\ Philippines; aglennvalerie@yahoo.com
}

\begin{abstract}
Objective: Electric energy consumption forecasting is important in electricity production planning, thus the aim of this study is to forecast the electricity consumption in every region in the Philippines for the next 3 years using the monthly data from 2013 up to 2018. Methods: The forecasting model used is the Markov-Chain Grey Model which has the merits of GM $(1,1)$ forecasting model. The two models are compared and the forecasting results for the year 2018 up to 2021 of both models show that the Markov-Chain Grey Model is more precise than GM $(1,1)$. Findings: In this study, the researchers deduce that highest electricity consumption was every second quarter of the year and during Christmas season. Moreover, lowest electricity consumption was every month of August. The reason for this significant fluctuation of demand in electricity were due to the damages caused by strong typhoon and heat index caused by phenomenal event El Nino. The predicted values of electricity consumption using Markov-Chain Grey Model in every region was forecasted to be constantly increasing in the next 3 years compared to actual monthly consumption from year 2013-2018. Mean Absolute Percentage

Error (MAPE) given by the Equation ${ }_{\text {MAPE }}=\frac{\sum_{i=1}^{n}\left|\frac{A_{i}-F_{i}}{A_{i}}\right| \times 100}{n}$, where $A_{t}$ is the actual value and $F_{t}$ is the forecast value, was used to measure the accuracy of forecast systems. MCGM gets an almost zero MAPE value that means that it is an indicator of good forecast of values, so, Markov model prediction was better that GM $(1,1)$. Applications/Improvements: For the government, this research can be used to raise awareness that demand for electricity will continue to increase over the next 3 years in order to be ready in future to prevent any power interruption. For the citizens, it can be used to disseminate knowledge for people in order to preserve energy as long as they can to reduce the likelihood of power reserve thinning.
\end{abstract}

Keywords: Demand, Forecasting Model, GM (1,1), Markov Chain Grey Model, Monthly Electricity Consumption

\section{Introduction}

Electricity is one of the most useful discoveries of mankind. In the Philippines, the electricity sector provides electricity through power generation, transmission and distribution. It serves as the main source of energy in Filipino households which enables the use of appliances, lighting and technologies and even for entertainment ${ }^{1}$.
In 2013, according to the Philippines Department of Energy, the Philippines consumed 75,266 Gigawatt-hours (GWh) of electricity ${ }^{2}$. Of this, $27.39 \%$ went to powering residential areas, $24.31 \%$ to commercial establishments and $27.46 \%$ to the industrial sector with $72.84 \%$ of electrical energy being consumed by Luzon, $14.75 \%$ by Visayas and $12.41 \%$ by. 
In year 2015, electricity sales and consumption increased remarkably by $6.7 \%$ from 77,261 GWh in 2014 to $82,413 \mathrm{GWh}^{3}$. The year 2016 is characterized by a significant increase in electricity consumption at $10 \%$ and peak demand at $8.7 \%$ attributed to several factors such as the increase in temperature and utilization of cooling equipment aggravated by the strong El Nino 4 . The residential and industrial sectors remained the major drivers of electricity consumption in the country while Luzon remained the largest on a per grid basis. Electricity consumption grew significantly from 82,413,213 MWh (2015) to $90,797,891 \mathrm{MWh}$ (2016). This year's growth level increased to $10.2 \%$ compared to the $6.7 \%$ growth from 2014-2015. This increase is primarily driven by the growth of residential consumption at $12.7 \%$ from 22,747,049 MWh (2015) to 25,631,254 MWh (2016) due to high requirements for cooling system. On a per grid basis, Mindanao's electricity consumption grew the highest at $12 \%$ boosted by the own-use consumption of newly operational and large coal-fired power plants 4 .

In 2017, electricity consumption in the Philippines rose $3.9 \%$ to 94.4 million MWh from 90.8 million MWh in $2016^{5}$. Although $74 \%$ of the total power generated is consumed in Luzon, consumption only grew 3.6\% annually. Electricity consumption in Visayas and Mindanao expanded at faster rates of $5.8 \%$ and $4.0 \%$, respectively.

In 2018, Electric cooperatives' sales in the first quarter jumped $11 \%$ amid increased demand nationwide, according to the National Electrification Administration (NEA) ${ }^{6}$. According to NEA Administrator Edgardo Masongsong, growth in power sales was driven by increased consumption across Luzon, Visayas and Mindanao. In the first three months of 2018, the 121 cooperatives under NEA's supervision sold a total of 4,924 GWh from 4,417 GWh in the same period in 2017. Residential consumers accounted for the biggest share of electricity consumed at $51 \%$ of total or $2,506 \mathrm{GWh}$, commercial establishments represented $24 \%$ of sales at 1,187 GWh while industrial customers had $18 \%$ or 881 GWh.

The scientific prediction for electricity consumption plays a significant role in drawing up an energy development policy and can serve as a decision-making basis and guarantee for plan in the next reconstruction of electric distribution network for each region of the Philippines. Precisely predicting for electricity consumption is important for planning and operation of electric power system. The common prediction methods include the GM $(1,1)$ and Markov-Chain Grey Model. Above those methods mentioned, each one has its own relative merits and disadvantages. In this paper, MarkovChain Grey Model was applied to predict electricity consumption.

In 1998, the study "Improved grey prediction models for the transpacific air passenger market" by. $\operatorname{In}^{7}$ has applied grey theory to develop time series $\operatorname{GM}(1,1)$ models for predicting traffic flows in the trans-Pacific air passenger market. The original GM $(1,1)$ models are improved by using residual modifications with Markovchain sign estimations. Results show that these models are more reliable by posterior checks and yields more accurate prediction than multiple regression models. The results indicate that the total number of air passengers in the trans-Pacific market will increase at an average annual growth rate of approximately $11 \%$ up to the year 2000 .

In year 2003, the study "Applying the Grey prediction model to the global integrated circuit industry" by ${ }^{8}$ examines the precision of the Grey forecasting model applied to samples based on demand and sales in the global integrated circuit industry. To increase the accuracy of GM $(1,1)$, three residual modification models are applied. Application of these models confirms that the Fourier series and Markov-chain approaches appear to perform better than GM $(1,1)$ model. Results show that the GM is better suited to short-term predictions than to mid and long term predictions. Meanwhile, the Markovchain residual modification model achieves reliable and precise results.

In 2005, He Y, and Huang M. published the paper entitled "A Grey-Markov Forecasting Model for the

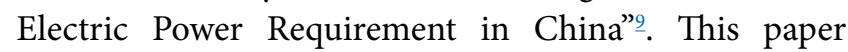
presents a Grey-Markov forecasting model for forecasting the electric power requirement in China. Results show that the forecasting precisions of Grey-Markov forecasting model from 2002 to 2004 are $99.42 \%, 98.05 \%$ and $97.56 \%$ and those of GM $(1,1)$ grey forecasting model are $98.53 \%$, $94.02 \%$ and $88.48 \%$. It shows that the Grey-Markov forecasting models have higher precision than GM $(1,1)$ grey forecasting model.

In 2007, the paper entitled "Predictive analysis on electric-power supply and demand in China" 10 by presents a Grey-Markov forecasting model to forecast the electric-power demand in China. This paper was based on historical data of the electric-power requirement 
from 1985 to 2001 in China and forecasted and analyzed the electric-power supply and demand in China by the Grey-Markov forecasting model. Results show that the Grey-Markov forecasting model is more suitable to forecast the electric-power demand.

In 2008, the paper entitled "Forecast of power generation for grid-connected photovoltaic system based on grey model and Markov chain" 11 the GreyMarkov forecast model was established in grid-connected photovoltaic generation. Once the solar generation is classified in order of the time and the size, the grey forecast model can be established and the generation fitting value and the residual value can be obtained which constructs Markov forecast model. Basing on the Grey forecast GM $(1,1)$ model and the stochastic processes Markov model, the deviation results of grey GM $(1,1)$ model was used as the deviation transfer probability matrix of Markov model forecast. In order to increase the forecast reliability, the calculation result of GM $(1,1)$ model was modified and limited in a forecast range which consisted of the interval and probability. The result shows that Grey-Markov model is more accurate than other forecast method.

Also, in 2008, published the paper entitled "Market Share Forecast of Electricity in City Residents' Energy Consumption based on Markov theory". Forecast have been done on market share of residential energy consumption items such as heating, cooking, cooling etc., with the use of Markov prediction theory and data from Qinhuangdao electricity market investigation. Forecast on market share at next term and at the equilibrium condition are accomplished which demonstrate the tendency of electricity as well other energy in residential energy consumption $\frac{12}{2}$.

In 2009, the paper entitled "Forecasting the turning time of stock market based on Markov-Fourier grey model" by $\underline{\underline{13}}$ presents an integration prediction method including Grey Model (GM), Fourier series and Markov state transition, known as Markov-Fourier Grey Model (MFGM), to predict the turning time of Taiwan Weighted Stock Index (TAIEX) for increasing the forecasting accuracy. The proposed Markov-Fourier Grey Model prediction approach uses the Grey Model to roughly predict the next datum from a set of the most recent data and the Fourier series to fit the residual errors produced by the Grey Model. Results show that the proposed approach MFGM has a higher forecasting accuracy than the other methods but it is only suitable for long-term operation.
In 2010, the study "Time series models (Grey-Markov, Grey Model with rolling mechanism and singular spectrum analysis) to forecast energy consumption in India" by ${ }^{14}$. Applies three-time series models, namely, Grey-Markov model, Grey Model with rolling mechanism and Singular Spectrum Analysis (SSA) to forecast the consumption of conventional energy in India. Grey-Markov model has been employed to forecast crude-petroleum consumption while Grey Model with rolling mechanism to forecast coal, electricity consumption and SSA to predict natural gas consumption. For short-term forecasting, the results clearly indicate that values forecasted by these models are very much close to the real values. A comparison of projected energy demand by the models presented in this study and the Planning Commission (2002-2007) shows that these time series models can be a viable alternative to project the future energy requirements.

In the year 2011, the paper entitled "Prediction of Rural Electricity Consumption Based on Grey-Markov Model" by shows that GM-Markov has higher prediction accuracy, comparing with the GM model and this predictive method has better precision and it is practical 15 .

In 2013, the paper entitled "Improvement of renewable energy supply forecasts: The case of Taiwan renewable industry" 16 presents an investigation of the annual supply value of the four renewable energies in Taiwan from 2005 to 2009. An improved grey GM $(1,1)$ model, using a technique that combines residual modification with the Markov chain model, is proposed. Results show that the Grey-Markov model could clearly improve the forecast accuracy of the original grey forecast model. It also shows that Grey-Markov forecasting model is the best for forecasting the annual output of the semiconductor and computer industries.

In the year 2013, published the paper entitled "Energy Demand Forecast of Iran's Industrial Sector Using Markov Chain Grey Model". The major purpose of this paper was to develop the prediction model of energy demand of industrial sector in Iran. Markov-Chain Grey Model (MCGM) has been proposed to forecast such energy demand. They used the statistics data of the energy consumption of industrial sector from 1990 to 2008. Grey Model (GM) and regression model has been compared with the proposed model to find its effectiveness. The comparison reveals that the MCGM model has higher precision than those of the GM and the regression. The MCGM is then used to forecast the annual energy demand 
of industrial sector in Iran up to the year 2020. The results provide scientific basis for the planned development of the energy supply of industrial sector in $\operatorname{Iran}^{17}$.

In 2014, the paper entitled "Combining Forecasts of Electricity Consumption in China with Time-varying weights updated by a High-order Markov-Chain Model" by. Proposes a time-varying-weight combining method called HM-TWA to predict the monthly electricity consumption in China. Out-of-sample tests of forecasting accuracy show the effectiveness of the proposed HM-TWA method to perform forecasting for one through eleven months ahead. The forecasting performance of HM-TWA has been compared with eight models (BPN, LSSVR, SARIMA, PSTM, PTTM, HWM, SA-WA and TSSE), results shows that HM-TWA has different degrees of improvement compared with other models for both onemonth-ahead and one-year-ahead forecasting according to the three criteria (RMSE, MAE and MAPE) ${ }^{18}$.

In 2015, the paper entitled "Forecasting China's energy demand and self-sufficiency rate by grey forecasting model and Markov model" by. In ${ }^{19}$ proposed an optimized single variable discrete grey forecasting model and the QP-Markov model for forecasting the total amount of energy production and consumption structures. The proposed models are used to simulate China's energy production and consumption during 2006-2011 and forecast its trends in 2015 and 2020. Results show that proposed models can effectively simulate and forecast the total amounts and structures of energy production and consumption. For the energy structures, the proportions of natural gas and other new energies in the production and consumption will continue to increase. The coal and other new energies can be self-sufficient in the forthcoming decade while the self-sufficiency rates of crude oil and natural gas will continue to drop. And by comparing with regression model, results show proposed model is a little better than regression in simulating and forecasting the case.

In the year 2017, the paper entitled "Analysis and Modeling for China's Electricity Demand Forecasting Based on a New Mathematical Hybrid Method" by was published. In this paper, a new mathematical hybrid method is proposed to forecast electricity demand. In line with electricity demand feature, the framework of joint-forecasting model is established and divided into two procedures: firstly, the modified GM $(1,1)$ model and Logistic model are used to make single forecasting. Then, the IOWHA operator is applied to combine these two single models and make joint-forecasting for China's electricity demand in 2016-2020. Forecasting results demonstrate that this new hybrid model is superior to both single-forecasting approaches and traditional joint-forecasting methods. Detailed forecasting-outcomes on electricity demand of China in 2016-2020 are discussed and displayed a slow-growth smoothly over the next fi, 1 years ${ }^{20}$.

In 2017, the paper entitled "Novel grey prediction model with nonlinear optimized time response method for forecasting of electricity consumption in China" by. In ${ }^{21}$ proposed an optimized hybrid GM $(1,1)$ model to improve prediction accuracy of EEC in short term, namely $\operatorname{IRGM}(1,1)$. As a comparison, three alternative grey prediction models are constructed to process these data. Results show that the errors of the proposed model decreases comparing with those of the three ones, and forecasting precision is improved.

In the year 2018, the paper entitled "A seasonal GM $(1,1)$ model for forecasting the electricity consumption of the primary economic sectors". $\operatorname{In}^{22}$ propose a Seasonal Grey Model (SGM $(1,1)$ model) based on the accumulation operators generated by seasonal factors. They use the proposed model to carry out an empirical analysis based on the seasonal electricity consumption data of the primary industries in China from 2010 to 2016.

The results from the SGM $(1,1)$ model are compared with those obtained using the grey model $(\operatorname{GM}(1,1))$, the particle swarm optimization algorithm combines with the grey model (PSO-GM(1,1) model), and the adaptive parameter learning mechanism based seasonal fluctuation GM $(1,1)$ model (APL-SFGM $(1,1)$ model). The results of the comparison show that the SGM $(1,1)$ model can effectively identify seasonal fluctuations in the electricity consumption of the primary industries and its prediction is more accurate. Results also show that the electricity consumption of China's primary industries is expected to continue increasing and show significant seasonal variation.

\subsection{Conceptual Framework}

\subsection{Statement of the Problem}

This study aims to answer the following questions:

- What are the behaviours of the graphs of monthly electricity consumption in each region in the Philippines from the year 2018 up to 2021 ? 


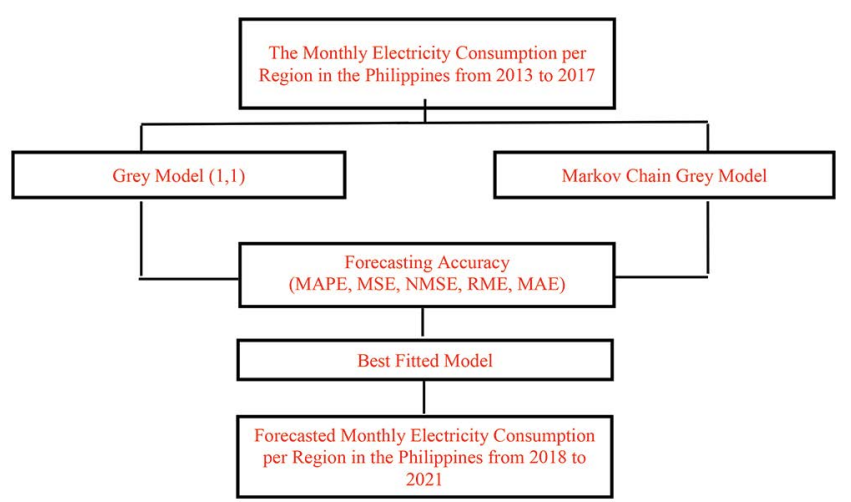

Figure 1. Conceptual framework of the study.

$>\quad$ National Capital Region (NCR).

$>$ Region I.

$>$ Cordillera Administrative Region (CAR).

$>$ Region II.

$>$ Region III.

$>$ Region IV-A.

$>$ Region IV-B.

$>$ Region V.

$>$ Region VI.

$>$ Region VII.

$>$ Region VIII.

$>$ Region IX.

$>$ Region X.

$>$ Region XI.

$>$ Region XII.

$>$ CARAGA.

$>$ Autonomous Region of Muslim Mindanao (ARMM).

- What are the mathematical models for the monthly electricity consumption in each region in the Philippines and which model is better?

- $\mathrm{GM}(1,1)$;

- Markov-Chain Grey Model;
- What will be the predicted values of electricity consumption in the Philippines for the year 2018 up to 2021 ?

\subsection{Scope and Limitation}

This study contains the monthly data of the electricity consumption in kilowatt-hour ( $\mathrm{kWh}$ ) of different regions in the Philippines from the year 2013 to 2017. The data gathered are from the Department of Energy (DOE). The data collected will be used to forecast the values of electricity consumption for 3 succeeding years from 2018 up to 2021.

\section{Method}

\subsection{Data Gathering}

The researchers gathered the data behaviors of the graphs of monthly electricity consumption in each region in the Philippines from the year 2018 up to 2021 from:

National Capital Region (NCR).

Region I.

Cordillera Administrative Region (CAR).

Region II.

Region III.

Region IV-A.

Region IV-B.

Region V.

Region VI.

Region VII.

Region VIII.

Region IX.

Region X.

Region XI.

Region XII.

CARAGA.

Autonomous Region of Muslim Mindanao (ARMM) 


\subsection{GM $(1,1)$ Forecasting Model}

In Grey model $(1,1)^{23}$. better known as $G M(1,1)$, has a time variable coefficient in which the model wasupdated into new data for model prediction or so-called Accumulated Generating Operation (AGO). The first step to do the forecasting using as $G M(1,1)$ is to set the original data into a sequence $x^{(0)}(k)$ with $k=1,2,3, \ldots, \mathrm{n}$ as follows:

$x^{(0)}=\left\{x^{(0)}(1), x^{(0)}(2), x^{(0)}(3), \ldots \ldots, x^{(0)}(\mathrm{n})\right\}$

The original data in Equation (1) is then arranged into a new sequence through one-time Accumulated Generating Operation (1-AGO), given by:

$x^{(1)}(k)=\left\{x^{(1)}(1), x^{(1)}(2), x^{(1)}(3) \ldots, x^{(1)}(\mathrm{n})\right\}$

Where and $x^{(0)}(1)=x^{(0)}(1)$ and $x^{(1)}(k)=\sum_{\mathrm{i}=1)}^{k} x^{(0)}(\mathrm{i})$, $k=1,2,3, \ldots, n$

The third step is to determine the value of, which is known as the Mean Generating Operation (MGO).

$$
z^{(1)}(k)=\frac{x^{(1)}(k)+x^{(1)}(k-1)}{2}(k=2,3,4, \ldots, n)
$$

The value of MGO in Equation (3) is used to solve the first order differential equation defined by:

$$
\frac{d x^{(1)}}{d t}+a x^{(1)}(k)=b k=2,3,4, \ldots, n
$$

Where is a developing coefficient and is a Grey input. By using the method of least squares, the parameters and can be obtained as follows:

$(a, b)^{T}=\left(B^{T} B\right)^{-1} B^{T} Y$

With $B=\left[\begin{array}{cc}-z^{(1)}(2) & 1 \\ -z^{(1)}(3) & 1 \\ \vdots & \vdots \\ -z^{(1)}(n) & 1\end{array}\right]$

And $\mathrm{Y}=\left\{x^{(0)}(2), x^{(0)}(3), x^{(0)}(4), \ldots, x^{(0)}(n)\right\}$

After determining the values of and, the solution of of Equation (4) was obtained as follows:

$$
\hat{x}^{(1)}(k)=\left(x^{(1)}(1)-\frac{b}{a}\right) e^{-a k}+\frac{b}{a}
$$

The prediction value using the model is which can be calculated by performing an Inverse Accumulated Generating Operation (IAGO) on Equation (6), obtaining

$$
\hat{x}^{(0)}(k+1)=\left(1-e^{a}\right)\left(x^{(0)}(1)-\frac{b}{a}\right) e^{-a k}
$$

Where $\hat{x}^{(0)}(1)=x^{(0)}(1)$

\subsection{Markov Chain Grey Model}

After obtaining the predicted value from $\operatorname{GM}(1,1)$, the next step is to apply the Markov Chain ${ }^{23}$ concept to the predicted value obtained from Equation (7). The algorithm of Markov Chain Grey model is as follows:

- Calculate the relative error between the predicted value from $\operatorname{GM}(1,1)$ and the original data:

Relative Error $(k)=\frac{x^{(0)}(k)-\hat{x}^{(0)}(k)}{x^{(0)}(k)} \times 100$

- Determine the number of states or conditions used to identify all possible conditions of a process or system.

- Each state has a boundary notated by

$$
\bigotimes_{j}=\left[\bigotimes_{j}, \bigotimes_{j},+\right], j=1,2,3, \ldots, m(9)
$$

where $\otimes_{j}$ is the boundary of the $j$-th state $\otimes_{(j-)}$,is the lower bound and $\otimes_{(j+)}$ is the upper bound of the $j$-th state.

- Determine the interval length of each state by calculating

$$
I_{(k)}=\frac{H-L}{r}
$$

Where $I_{(k)}$ is the kth interval, $\mathrm{H}$ is the highest value of relative error, $\mathrm{L}$ is the lowest value of relative error, and $\mathrm{r}$ is the number of states. Therefore, it produces the boundary of each state as follows:

$$
\begin{aligned}
& \left.\bigotimes_{1}=\left[\mathrm{L}, I_{(1)}\right] ; \bigotimes_{2}=\left[I_{(2)}, I_{(3)}\right], \ldots, \bigotimes_{\mathrm{n}}=\left[I_{(n)}\right), H\right], i= \\
& 1,2, \ldots, n
\end{aligned}
$$

- Form a transition probability matrix using the Markov Chain concept as follows:

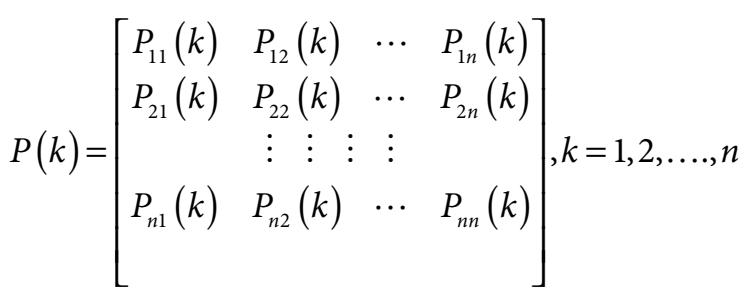

$P(k)$ is the $k$ step transition probability, while $P_{i j}$ denotes the probability that if the process is in state $i$ at time $t$, then it will switch to state $j$ at time $t+1$. Therefore, 
$P_{i j} \geq 0$ for all $i, j\{0,1,2, ..\} \sum_{j=0}^{\infty} P_{i j}=1$ and for all $i\{0,1,2, \ldots\}$.

Determine the next state for the prediction based on the vector row on the transition probability matrix by time (based on the nearest time to the predicted time), then summing up the probability values obtained according to their respective states. The state that has the greatest probability is the state that is most likely for the time to be predicted.

After determining the state that has the greatest probability, then the boundary of the state will be used to predict the future data using equation below:

$$
\hat{x}(k)=\hat{x}^{(0)}(k) \times\left(1+\frac{\otimes_{(j-)}+\otimes_{(j+)}}{2} \%\right)
$$

\subsection{Forecasting Accuracy}

\subsubsection{Mean Absolute Percentage Error}

The mean absolute percentage error (MAPE) $)^{24}$ is a statistical measure of how accurate a forecast system is. It measures this accuracy as a percentage, and can be calculated as the average absolute percent error for each time period minus actual values divided by actual values. Where $A_{t}$ is the actual value and $F_{t}$ is the forecast value, this is given by:

$$
M A P E=\frac{\sum_{i=1}^{n}\left|\frac{A_{i}-F_{i}}{A_{i}}\right| \times 100}{n}
$$

\subsubsection{Mean Absolute Error}

The Mean Absolute Error (MAE) ${ }^{25}$ is the average of all absolute errors. The formula is:

$$
\text { MAE }=\frac{\sum_{i=1}^{n}\left|F_{i}-A_{i}\right|}{n}
$$

\subsubsection{Mean Square Error}

The Mean Squared Error ${ }^{26}$ tells you how close a regression line is to a set of points. It does this by taking the distances from the points to the regression line (these distances are the "errors") and squaring them.

The squaring is necessary to remove any negative signs. It also gives more weight to larger differences.
It's called the mean squared error as you're finding the average of a set of errors.

$$
M S E=\frac{\sum_{i=1}^{n}\left(A_{i}-F_{i}\right)^{2}}{n}
$$

\subsubsection{Root Mean Square Error}

Root Mean Square Error (RMSE) $)^{27}$ is the standard deviation of the residuals (prediction errors). Residuals are a measure of how far from the regression line data points are; RMSE is a measure of how spread out these residuals are. In other words, it tells you how concentrated the data is around the line of best fit. Root mean square error is commonly used in climatology, forecasting, and regression analysis to verify experimental results.

$$
R M S E=\sqrt{\frac{\sum_{i=1}^{n}\left(A_{i}-F_{i}\right)^{2}}{n}}
$$

\subsubsection{Normalized Mean Square Error}

The NMSE (Normalized Mean Square Error) ${ }^{28}$ is an estimator of the overall deviations between predicted and measured values. It is defined as:

$$
\begin{aligned}
& \text { NMSE }=\frac{\sum_{i=1}^{n}\left(A_{i}-F_{i}\right)^{2}}{n(\bar{A} \times \bar{F})} \\
& \bar{A}=\frac{1}{n} \sum A_{t} \\
& \bar{F}=\frac{1}{n} \sum F_{t}
\end{aligned}
$$

Where $A_{t}$ is the actual data, $F_{t}$ is the predicted value and is the total number of data.

\section{Results and Discussions}

\subsection{Behavior of the Graph per Region}

In this section, the researchers provided graphical representations of the actual data of the monthly electricity consumption of every region in the Philippines from the year 2013 up to 2018 that were obtained from the Department of Energy. Moreover, a discussion for every data gathered per region was interpreted. 


\subsubsection{NCR}

In Figure 2, the electricity consumption in NCR reaches its minimum in the month of august in every year. The graph goes down when approaching the months of June, July, and August and goes up when approaching the months of April, May, and December. The reason for this is that electricity demand is high during summer season and is low during rainy season. As heat index increases, energy consumption also increases ${ }^{29}$. Electricity consumption is high in the month of December because of the high usage of energy using Christmas decorations such as Christmas lights. April 2017 has the highest monthly electricity consumption on NCR. The National Grid Corporation of the Philippines (NGCP) recorded its highest electricity consumption for the months of April and May for the year 2017 because of the dry season ${ }^{30}$.

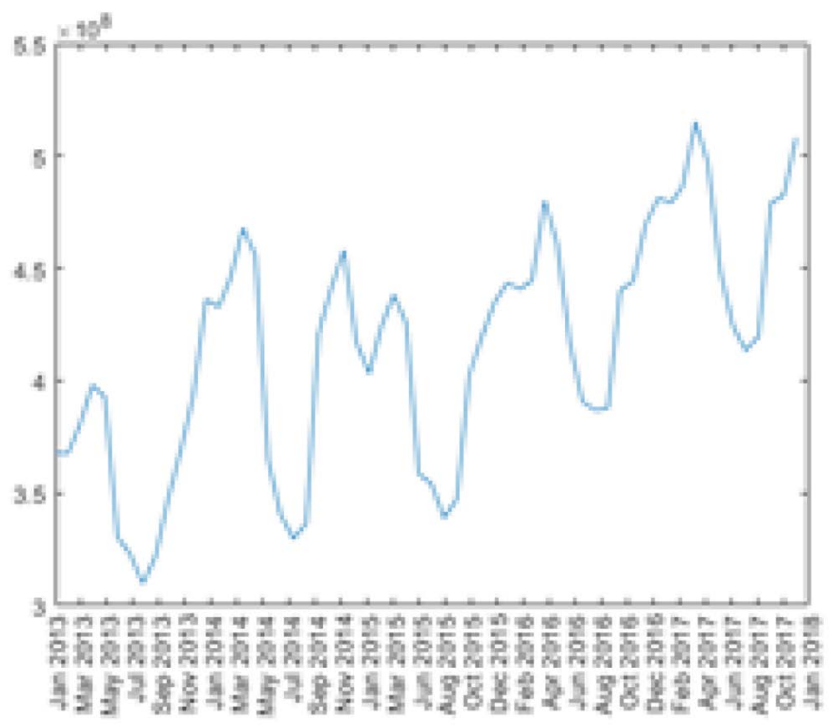

Figure 2. Monthly electricity consumption of NCR from Jan. 2013-Dec 2017.

\subsubsection{Region I}

In Figure 3, the monthlyelectricity consumption goes up every March, April, May, and December of the year. The electricity consumption reaches its lowest value in the month of August for each year. The reason for this is that electricity demand is high during summer season and is low during rainy season. As heat index increases, energy consumption also increases ${ }^{29}$. Moreover, electricity consumption is high in the month of December because of the high usage of energy using Christmas decorations such as Christmas lights. April 2017 has the highest monthly electricity consumption on Region I. The
National Grid Corp. of the Philippines (NGCP) recorded its highest electricity consumption for the months of April and May for the year 2017 because of the dry season ${ }^{30}$.

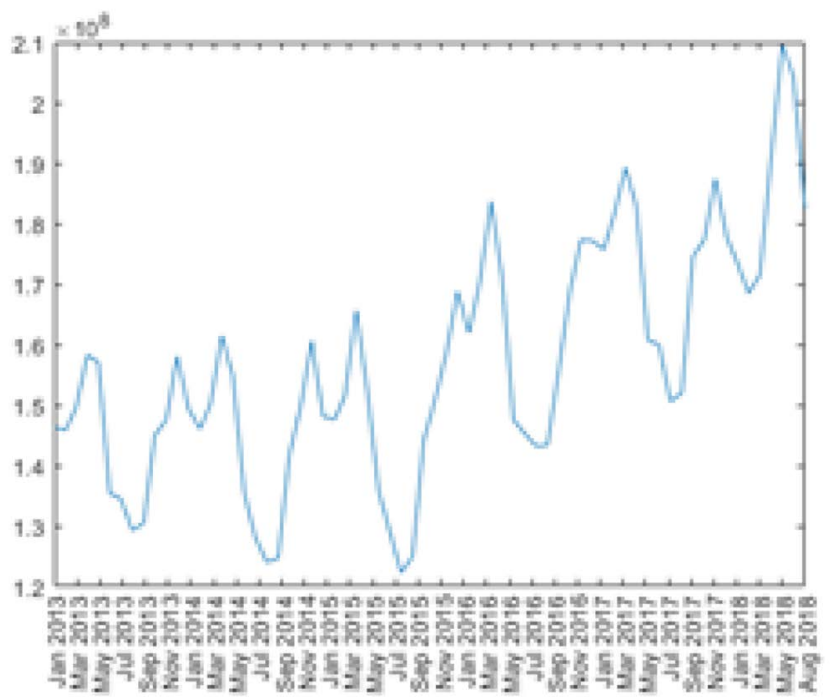

Figure 3. Monthly electricity consumption of Region I from Jan. 2013-Dec 2017.

\subsubsection{CAR}

In Figure 4, the monthly electricity consumption each year increases because the country's demand for power increases as the population and economy continues to grow.The graph's reaches its peak at the month of April in every year. The electricity consumption in the month of April every year is high because of it being in the summer season. As heat index increases, energy consumption also increases ${ }^{29}$. April 2017 has the highest monthly electricity consumption for the Cordillera Administrative Region (CAR). The National Grid Corp. of the Philippines (NGCP) recorded its highest electricity consumption for the months of April and May for the year 2017 because of the dry season ${ }^{30}$.

\subsubsection{Region II}

In Figure 5, the graph shows a great increase during the months of March, April, and May. A decrease is observed on the months of June, July, August, and up to September of each year. The reason for this is that electricity demand is high during summer season and is low during rainy season. As heat index increases, energy consumption also increases ${ }^{29}$. April 2017 has the highest monthly electricity consumption on Region II. The National Grid Corp. of the Philippines (NGCP) recorded its highest electricity 


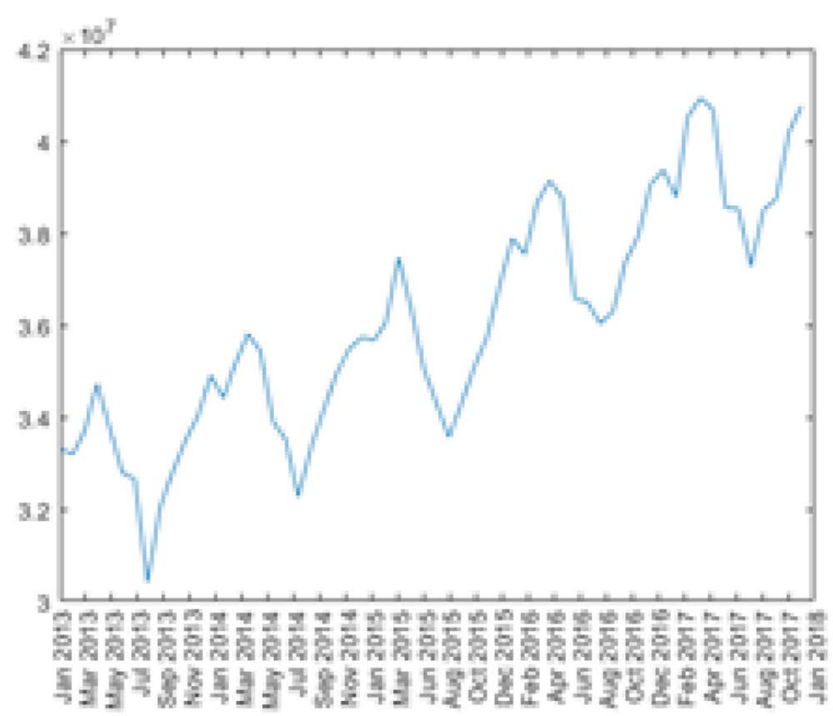

Figure 4. Monthly electricity consumption of CAR from Jan. 2013-Dec 2017.

consumption for the months of April and May for the year 2017 because of the dry season ${ }^{30}$.

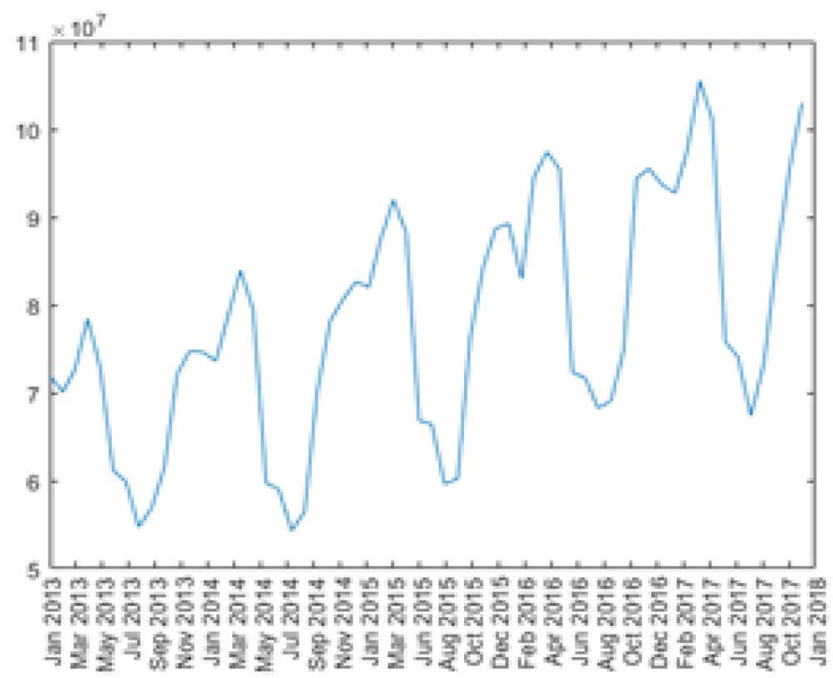

Figure 5. Monthly electricity consumption of Region II from Jan. 2013-Dec. 2017.

\subsubsection{Region III}

In Figure 6, the graph goes down when approaching the months of June, July, and August and goes up when approaching the months of April, May, and December. The reason for this is that electricity demand is high during summer season and is low during rainy season. As heat index increases, energy consumption also increase ${ }^{29}$. Also, electricity consumption is high in the month of December because of the high usage of energy using
Christmas decorations such as Christmas lights. April 2017 has the highest monthly electricity consumption on Region III. The National Grid Corp. of the Philippines (NGCP) recorded its highest electricity consumption for the months of April and May for the year 2017 because of the dry season ${ }^{30}$.

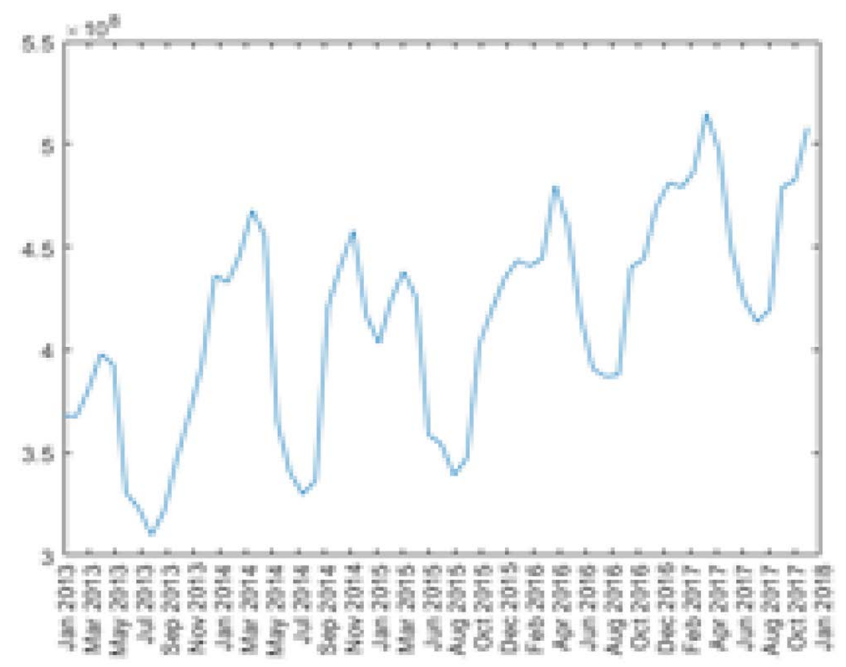

Figure 6. Monthly electricity consumption of Region III from Jan. 2013-Dec. 2017.

\subsubsection{Region IV-A}

In Figure 7, the graph goes up when approaching the months of April, May, and December and the graph goes down when approaching the months of July, August, and September. The reason for this is that electricity demand is high during summer season and is low during rainy season. As heat index increases, energy consumption also increases ${ }^{29}$. Also, electricity consumption is high in the month of December because of the high usage of energy using Christmas decorations such as Christmas lights.

April 2017 has the highest monthly electricity consumption on Region IV-A. The National Grid Corp. of the Philippines (NGCP) recorded its highest electricity consumption for the months of April and May for the year 2017 because of the dry season ${ }^{30}$.

\subsubsection{Region IV-B}

In Figure 8, the graph shows an increase when approaching the months of April, May, and December while the graph decreases when approaching the months of June, July, August, and September. The reason for this is that electricity demand is high during summer season and is low during rainy season. As heat index increases, 


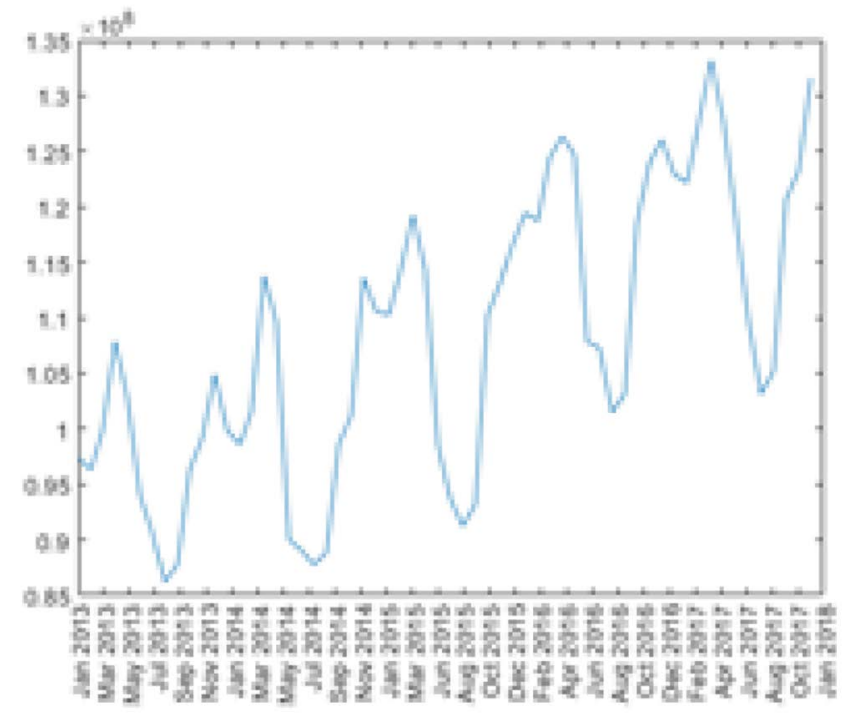

Figure 7. Monthly electricity consumption of Region IV-A from Jan. 2013-Dec. 2017.

energy consumption also increases ${ }^{29}$. Also, electricity consumption is high in the month of December because of the high usage of energy using Christmas decorations such as Christmas lights. April 2017 has the highest monthly electricity consumption on Region IV-B The National Grid Corp. of the Philippines (NGCP) recorded its highest electricity consumption for the months of April and May for the year 2017 because of the dry season ${ }^{30}$.

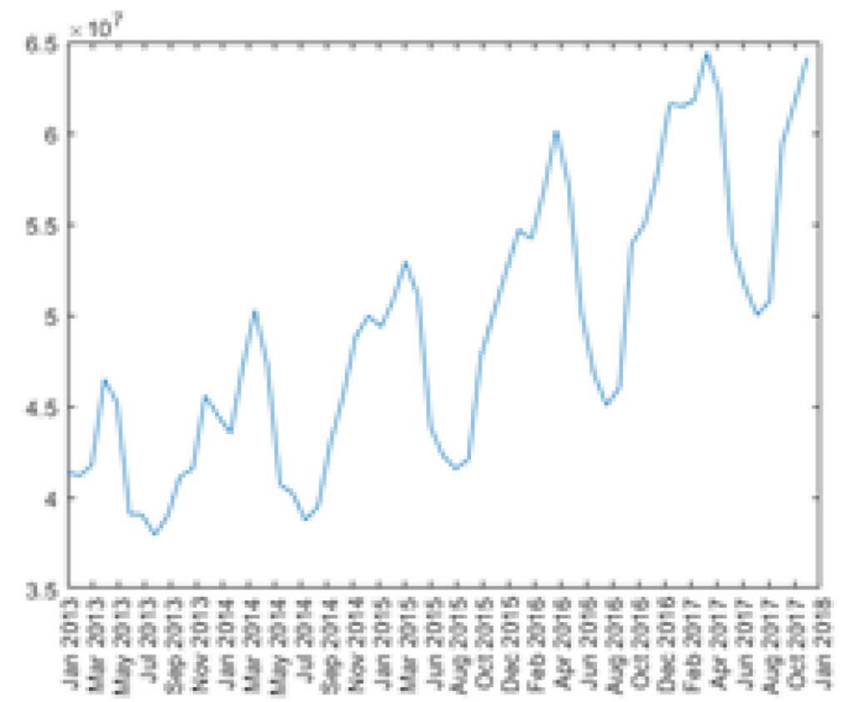

Figure 8. Monthly electricity consumption of Region IV-B from Jan. 2013-Dec. 2017.

\subsubsection{Region $V$}

In Figure 9, the graph shows an increasing function, reaching its peak every April of each year. The reason for this is that electricity demand is high during summer season, as heat index increases, energy consumption also increases $^{29}$. But a sudden fluctuation happened when the graph approaches the month of August 2017. The graph goes up again after the month of August 2017. April 2017 has the highest monthly electricity consumption on Region V. The National Grid Corp. of the Philippines (NGCP) recorded its highest electricity consumption for the months of April and May for the year 2017 because of the dry season ${ }^{30}$.

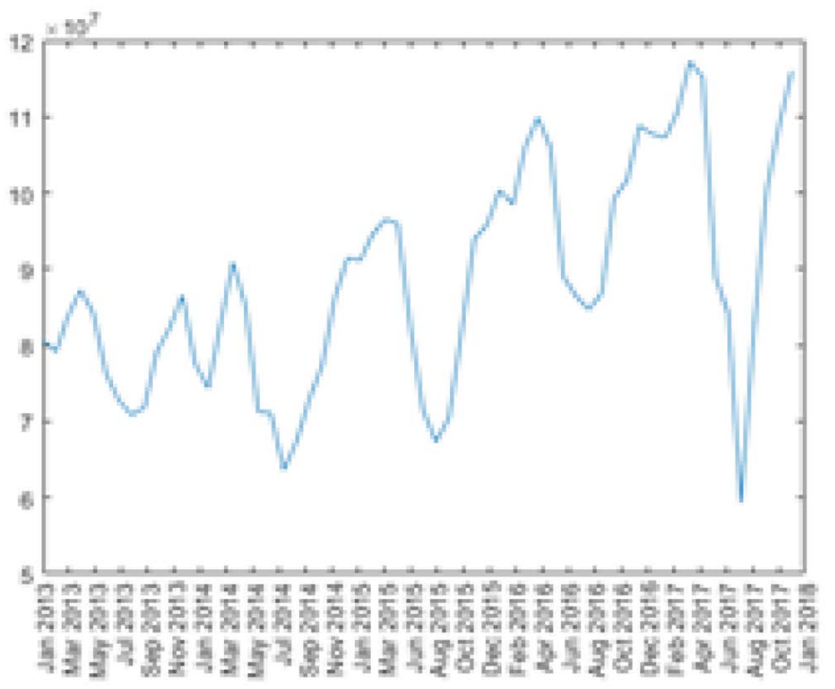

Figure 9. Monthly electricity consumption of Region V from Jan. 2013-Dec. 2017.

\subsubsection{Region VI}

In Figure 10, the graph goes up when approaching the months of April and December, and goes down when approaching the months of August. The reason for this is that electricity demand is high during summer season and is low during rainy season. As heat index increases, energy consumption also increases ${ }^{29}$. Also, electricity consumption is high in the month of December because of the high usage of energy using Christmas decorations such as Christmas lights. April 2017 has the highest monthly electricity consumption on Region VI. The National Grid Corp. of the Philippines (NGCP) recorded its highest electricity consumption for the months of April and May for the year 2017 because of the dry season ${ }^{30}$.

\subsubsection{Region VII}

In Figure 11, the graph increases when approaching the months of April, May, and December and decreases when approaching the month of August. April 2017 has the highest monthly electricity consumption on Region 


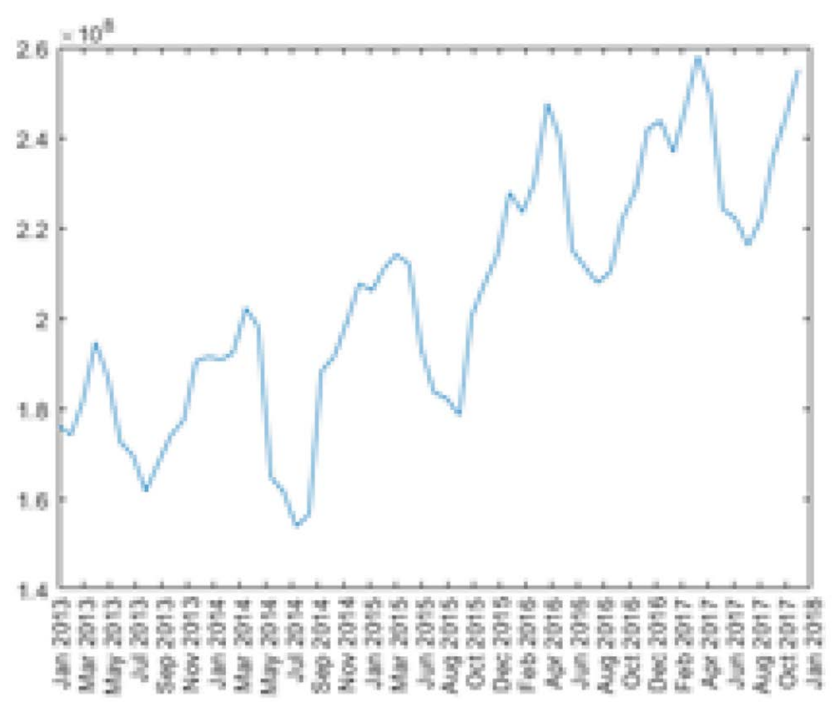

Figure 10. Monthly electricity consumption of Region VI from Jan. 2013-Dec. 2017.

VII. The reason for this is that electricity demand is high during summer season and is low during rainy season. As heat index increases, energy consumption also increases ${ }^{29}$. Also, electricity consumption is high in the month of December because of the high usage of energy using Christmas decorations such as Christmas lights. April 2017 has the highest monthly electricity consumption on Region VI. The National Grid Corp. of the Philippines (NGCP) recorded its highest electricity consumption for the months of April and May for the year 2017 because of the dry season ${ }^{30}$.

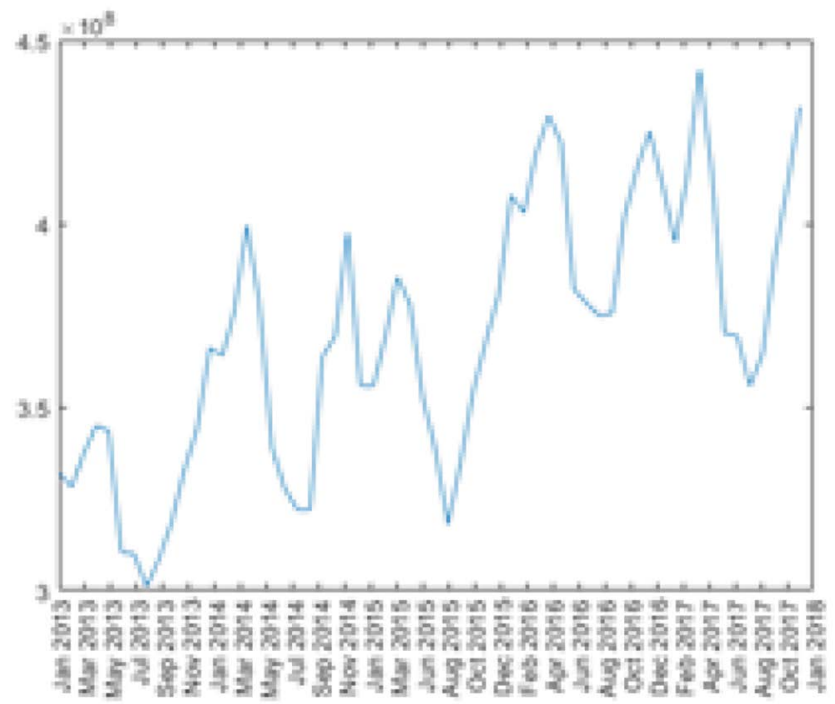

Figure 11. Monthly electricity consumption of Region VII from Jan. 2013-Dec. 2017.

\subsubsection{Region VIII}

In Figure 12, the graph reaches its maximum every April of each year, while the graph reaches its minimum every August of each year. This is the results of every typhoon occurred in the area of Region 8. Also, in 2013, the First Gen Hydro Power Corporation was markedly lower by PHP 2.25 billion and was the major driver of the PHP 2.71 billion decrease in our consolidated revenues ${ }^{31}$. April 2017 has the highest monthly electricity consumption on Region VIII. The reason was the power situation in the Visayas grid has been generally stable due to the additional capacities that went on commercial operation ${ }^{32}$.

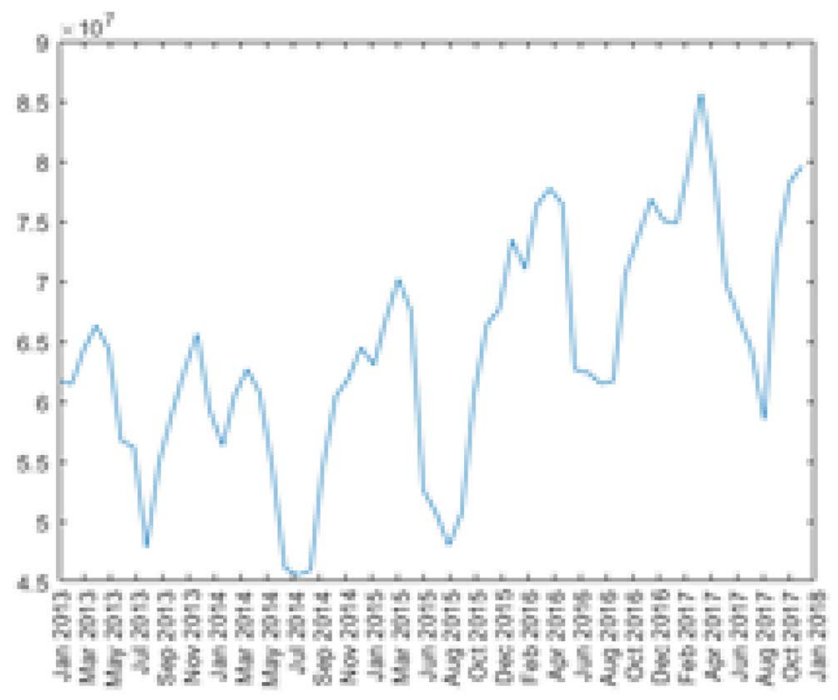

Figure 12. Monthly electricity consumption of Region VIII from Jan. 2013-Dec. 2017.

\subsubsection{Region IX}

In Figure 13, the graph goes up when approaching the months of April, May, and December, while the graph goes down when approaching the month of August. April 2016 and April 2017 has the highest monthly electricity consumption on Region IX. In 2014, the demand for power supply in ZAMSURECO l's franchise area is steadily increasing. Furthermore, the National Power Corporation/Power Sector Assets and Liabilities Management Corporation, the supplier of the Bulk of ZAMSURECOl's requirements, has significantly reduced its supply commitments to ZAMSURECO I by about one-third ${ }^{33}$. In 2015, the power shortage in Mindanao has remained a critical problem for ZAMSURECO II which continues to suffer rotating brownouts in the grid ${ }^{34}$. 


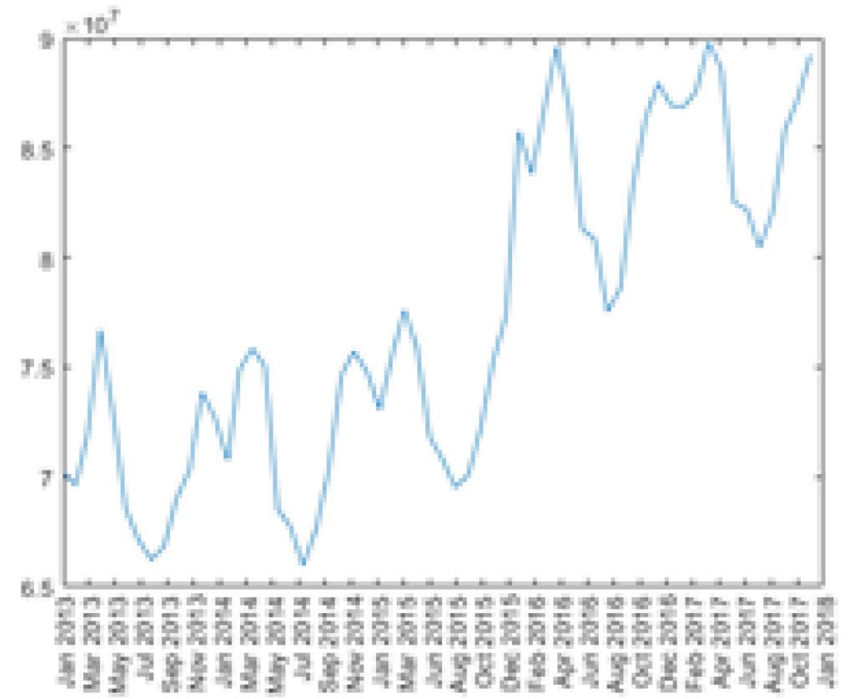

Figure 13. Monthly electricity consumption of Region IX from Jan. 2013-Dec. 2018.

\subsubsection{Region $X$}

In Figure 14, the monthly electricity consumption increases on the months of April, May, and December while it decreases when approaching the months of August and September. The electric cooperative was presently implementing two sets of daily rotating brownouts 14 hours daily due to the power shortage, which was mainly attributed to the declining generation capacity of the National Power Corporation's (NPC) hydroelectric plants ${ }^{35}$. The summer of 2013 has reached an all-time high hitting the mid-30's, as a result of every electric companies to increase their power supply. April 2016 has the highest monthly consumption on Region $\mathrm{X}$ but a sudden fluctuation happens when the graph approaches August and September 2017 $\stackrel{36}{\text {. }}$

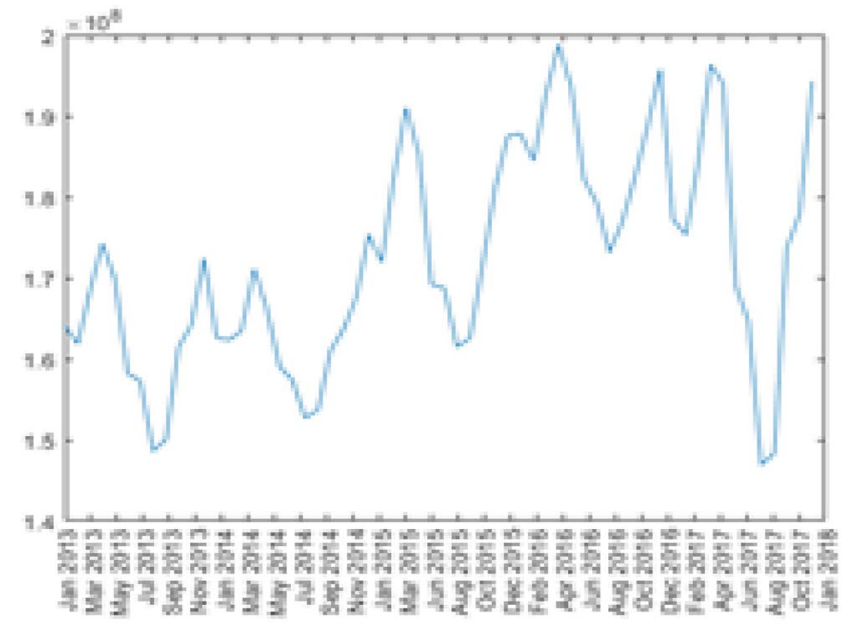

Figure 14. Monthly electricity consumption of Region X from Jan. 2013-Dec. 2017.

\subsubsection{Region XI}

In Figure 15, the graph goes up when approaching the months of April, May, and December while the graph goes down when approaching the months August and September. April 2017 has the highest monthly consumption on Region XI ${ }^{37}$. In 2014, power supply is not sufficient to meet the total power requirement and can no longer provide the required energy. PSALM has reduced the yearly supplied energy to an average of $36 \% \frac{38}{\text {. }}$.

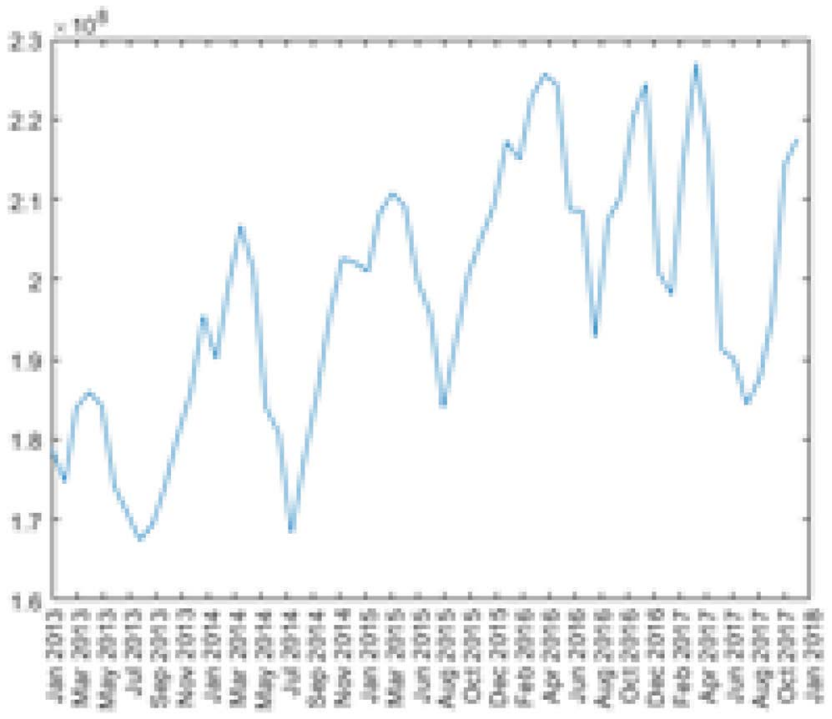

Figure 15. Monthly electricity consumption of Region XI from Jan. 2013-Dec. 2017.

\subsubsection{Region XII}

In Figure 16, the graph reaches its peak every April of each year and reaches its minimum every August. April 2016 has the highest monthly consumption on Region XII. In the year 2013 the daily rotational brownouts in Gen. Santos City and parts of South Cotabato and Sarangani provinces have stretched to seven hours due to a major power load cut imposed by the National Grid Corp. of the Philippines (NGCP) after the toppling of its transmission tower in North Cotabato ${ }^{38}$.As a result of contracts to other suppliers, the problems in brown-out was resolved by increasing the electric bill for the residential consumer ${ }^{39}$.

\subsubsection{CARAGA}

In Figure 17, the graph shows an increasing function, reaching its peak at every April of each year, and getting its minimum at every August of each year. April 2017 has the highest monthly consumption on CARAGA. ASELCO's power supply was not sufficient to meet its total power 


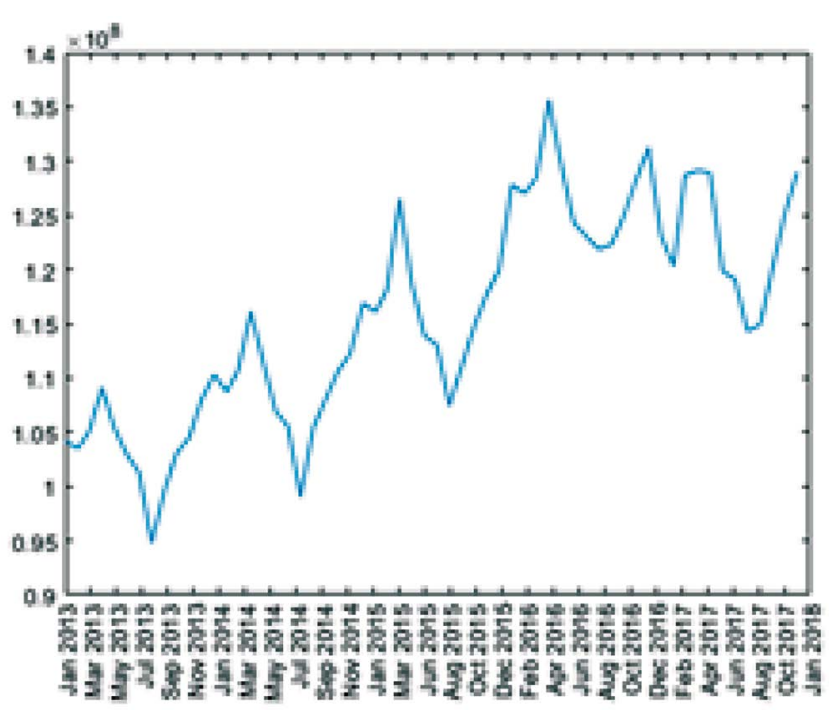

Figure 16. Monthly electricity consumption of Region XII from Jan. 2013-Dec. 2017.

requirements. ASELCO's power requirements stand at about $23 \mathrm{MW}$ but its available supply amounts to about 12 MW. Thus, there is a very significant shortage of $11 \mathrm{MW}$, almost half of its power requirements. As a result, the electricity consumers and local businesses in ASELCO's franchise area suffer from daily rotational brownouts of four (4) to five (5) hours. The solution was to increase the distribution of power supplies and rates ${ }^{40}$.

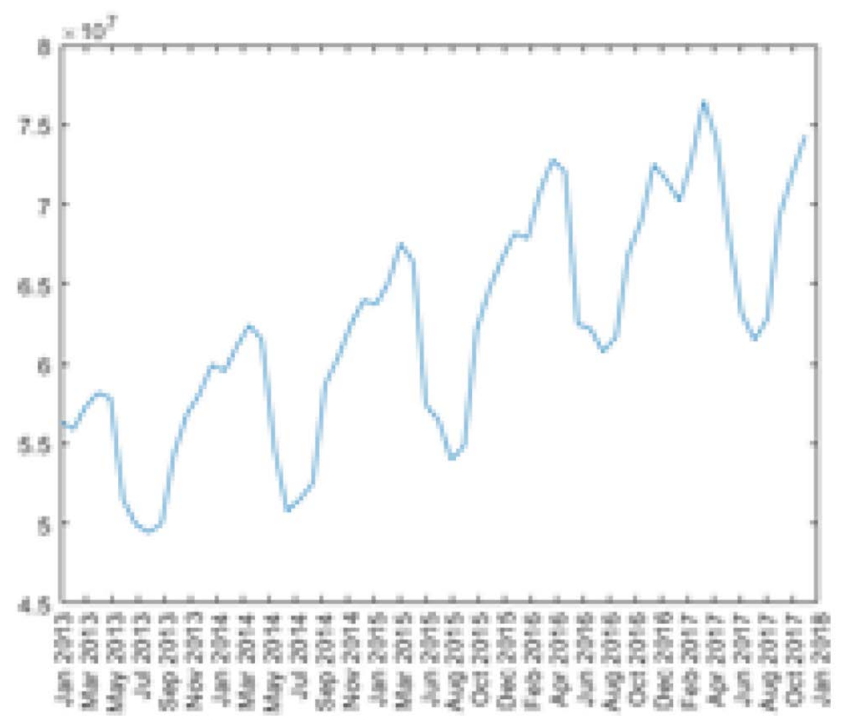

Figure 17. Monthly electricity consumption of CARAGA from Jan. 2013- Dec. 2017.

\subsubsection{ARMM}

In Figure 18, the graph reaches its maximum at July 2017 and reaches its minimum at September 2013. July
2017 has the highest monthly consumption on ARMM. In Mindanao, the problematic ECs are the Tawi-Tawi Electric Cooperative Inc. (Tawelco), Basilan Electric Cooperative Inc. (Baselco), Sulu Electric Cooperative Inc. (Suleco) and the Lanao Sur Electric Cooperative Inc. The Mindanao cooperatives are the biggest thorn on Petilla's side with their huge amount of debts ${ }^{40}$.

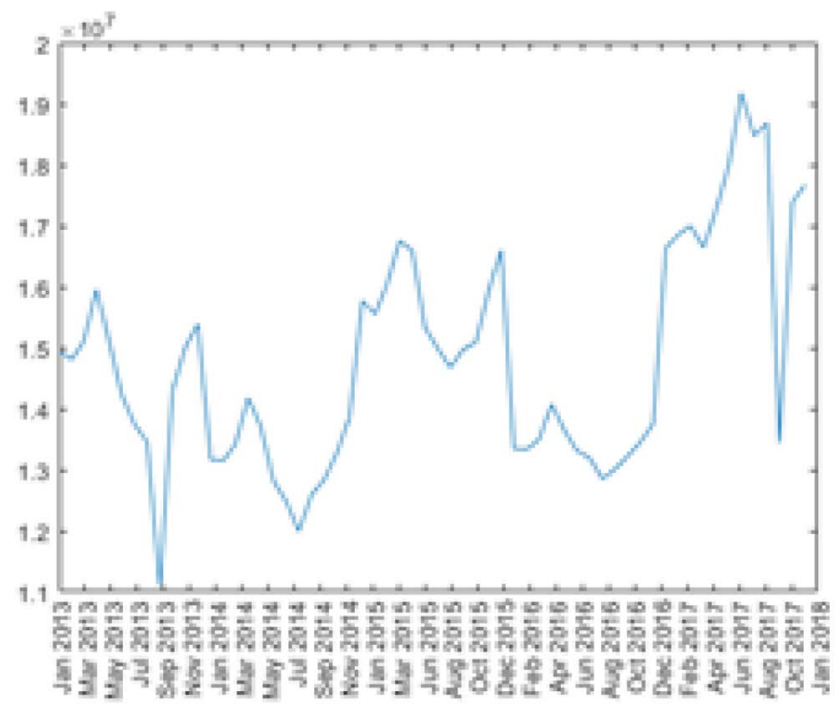

Figure 18. Monthly electricity consumption of ARMM from Jan. 2013- Dec. 2017.

\subsection{Result of the Markov-Chain Grey Model}

In this section, the process of $\operatorname{GM}(1,1)$ and MarkovChain Grey Model were used for the monthly electricity consumption of every region in the Philippines in the year 2013 up to 2018. Also, a comparison between the actual values using the Grey Model $(1,1)$ and Markov-Chain Grey Model of the monthly electricity consumption of the National Capital Region was presented to determine which model is best fitted based on the results of the forecasting errors. A step by step procedure was given to obtain the forecasted values as mentioned in the methodology.

The actual/original data of monthly electricity consumption for NCR, $\hat{x}^{(0)}$, is shown in Table 1:

Obtaining $x^{(1)}$ using 1-AGO(one-time accumulated generating operation), where

$$
x^{(1)}(k)=\sum_{i=1}^{k} x^{(0)}(i), k=1,2,3, \ldots, n
$$

Then the value for $x^{(1)}$ is shown in Table 2: 
Using the grey differential equation of GM $(1,1), x^{(0)}$ $(k)+a x^{(1)}(k)=b, k=2,3 \ldots n$, its whitening equation is obtained:

$$
\frac{d x^{(1)}}{d t}+a x^{(1)}(k)=b
$$

Let $\hat{\mathrm{u}}$ be the parameters vector, $\hat{\mathrm{u}}=(\hat{\mathrm{a}}, \hat{b})^{T}=\left(B^{T} B\right)^{-1}$ $\mathrm{B}^{T} \mathrm{Y}_{N^{p}} B$, denotes the accumulated matrix and $Y$ is the constant vector, so $a$ and $b$ can be obtained by using least square method. Where

$$
\begin{aligned}
& B=\left(\begin{array}{cc}
-z^{(1)}(2) & 1 \\
-z^{(1)}(3) & 1 \\
\vdots & \vdots \\
-z^{(1)}(n) & 1
\end{array}\right), Y_{N}=\left(\begin{array}{cc}
x^{(0)} & (2) \\
x^{(0)} & (3) \\
\vdots & \\
x^{(0)} & (n)
\end{array}\right) \\
& , z^{(1)}(k)=\frac{x^{(1)}(k)+x^{(1)}(k-1)}{2}(k=2,3,4, \ldots n)
\end{aligned}
$$

Then,

$$
\begin{aligned}
& B=\left(\begin{array}{cc}
-551868325.5575 & 1 \\
-925841525.875561 & 1 \\
\vdots & \vdots \\
-27499102026.2865 & 1
\end{array}\right), Y_{N}=\left(\begin{array}{c}
367683635.308333 \\
380262765.32779 \\
\vdots \\
561810112.98
\end{array}\right) \\
& \Rightarrow\left(B^{T} B\right)^{-1} B^{T} Y_{N}=\left(\begin{array}{r}
-0.00484671916777 \\
356962774.120313
\end{array}\right)
\end{aligned}
$$

Therefore, $a=-0.00484671916777$ and $=3569627$ 74.120313 .

The solution of the whitening equation of $\operatorname{GM}(1,1)$ can be obtained as follows:

$$
\hat{x}^{(1)}(k+1)=\left(x^{(0)}(1)-\frac{b}{a}\right) e^{(-a k)}+\frac{b}{a}
$$

Then,

$$
\begin{aligned}
& \hat{x}^{(1)}(k+1)= \\
& \left(368026507.903333-\left(\frac{356962774.12}{-0.0048}\right)\right) \\
& e^{-(-0.0048)(k)}+\frac{356962774.12}{-0.0048}
\end{aligned}
$$

Next, applying the Inverse Accumulated Generating Operation IAGO), we have

$$
\hat{x}^{(0)}(k+1)=\left(1-e^{a}\right)\left(x^{(0)}(1)-\frac{b}{a}\right) e^{-a k}
$$

Therefore, the mathematical model for GM $(1,1)$ is:

$$
\begin{aligned}
& \hat{x}^{(0)}(k+1)=\left(1-e^{-0.0048}\right) \\
& \left(368026507.903333-\left(\frac{356962774.12}{-0.0048}\right)\right) \\
& e^{-(-0.0048)(k)}
\end{aligned}
$$

The values for $\hat{x}^{(1)}(k+1)$ and $\hat{x}^{(0)}(k+1)$ are shown in Table 3:

Calculate now the relative error between the predicted value from $\mathrm{GM}(1,1)$ and the original data:

$$
\text { Relative Error }(k)=\frac{x^{(0)}(k)-\hat{x}^{(0)}(k)}{x^{(0)}(k)} \times 100
$$

The Table 4 shows the relative error for each $k=$ $1,2, \ldots, 66$

Now, we will divide $\hat{x}^{(0)}$ into $n$ states according to the relative error and determine the interval length of each state by dividing the difference of the highest value of relative error and lowest value of relative error to the number of states. We denote the states of as $\hat{x}^{(0)}$ as $\bigotimes_{j}=\left[\bigotimes_{j}, \bigotimes_{j+}\right]$

Now, the interval length of each is:

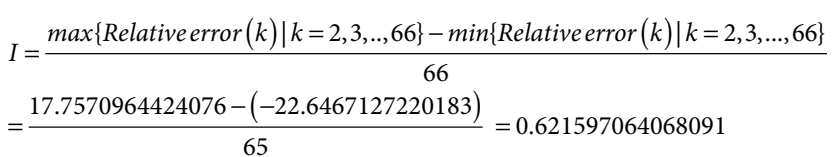

Where I is the interval length of each state.

Then the following interval for each state is shown in Table 5:

We will now establish the transition probability from $\bigotimes_{i}$ to $\bigotimes_{j}$ as follows:

$$
P_{i j}(k)=\frac{n_{i j}(k)}{n_{i}}, i=1,2,3, \ldots, n,
$$


Where $P_{i j}(k)$ is the transition probability of state $\otimes_{j}$ transferred from state $\bigotimes_{i}$ for $\mathrm{k}$ steps, $\mathrm{k}$ is the number of transition steps each time, $n_{i}$ is the number of data in state $\bigotimes_{i} n_{i j}(k)$ is the number of original data of state $\bigotimes_{j}$ transferred from state $\bigotimes_{i}$ for $\mathrm{k}$ steps. Then the transition probability matrix can be expressed as follow:

$$
P(k)=\left(\begin{array}{cccc}
P_{11}(k) & P_{12}(k) & \ldots & P_{1 n}(k) \\
P_{21}(k) & P_{22}(k) & \ldots & P_{2 n}(k) \\
\vdots & \vdots & \vdots & \vdots \\
P_{n 1}(k) & P_{n 2}(k) & \ldots & P_{n n}(k)
\end{array}\right)
$$

$\mathrm{P}(1)=$
Since 65 states are divided, then the latest 65 months of data near to prediction time are selected to make state prediction table. The transition steps are defined as 1,2 , $3, \ldots, 65$.

The sum of all transition probability from any state to state 65 is maximal, and then the relative error of July2018 is in the state $43:(3.06,3.68]$, the forecast value of $\operatorname{GM}(1,1)$ can be calculated using the formula: $\hat{x}^{(0)}(k+1)=\left(1-e^{a}\right)\left(x^{(0)}(1)-\frac{b}{a}\right) e^{-a k}$, the forecast value of July 2018 obtained by $\operatorname{GM}(1,1)$ is $492,787,024.62$, so the forecast value obtained by Markov Chain Grey Model is:

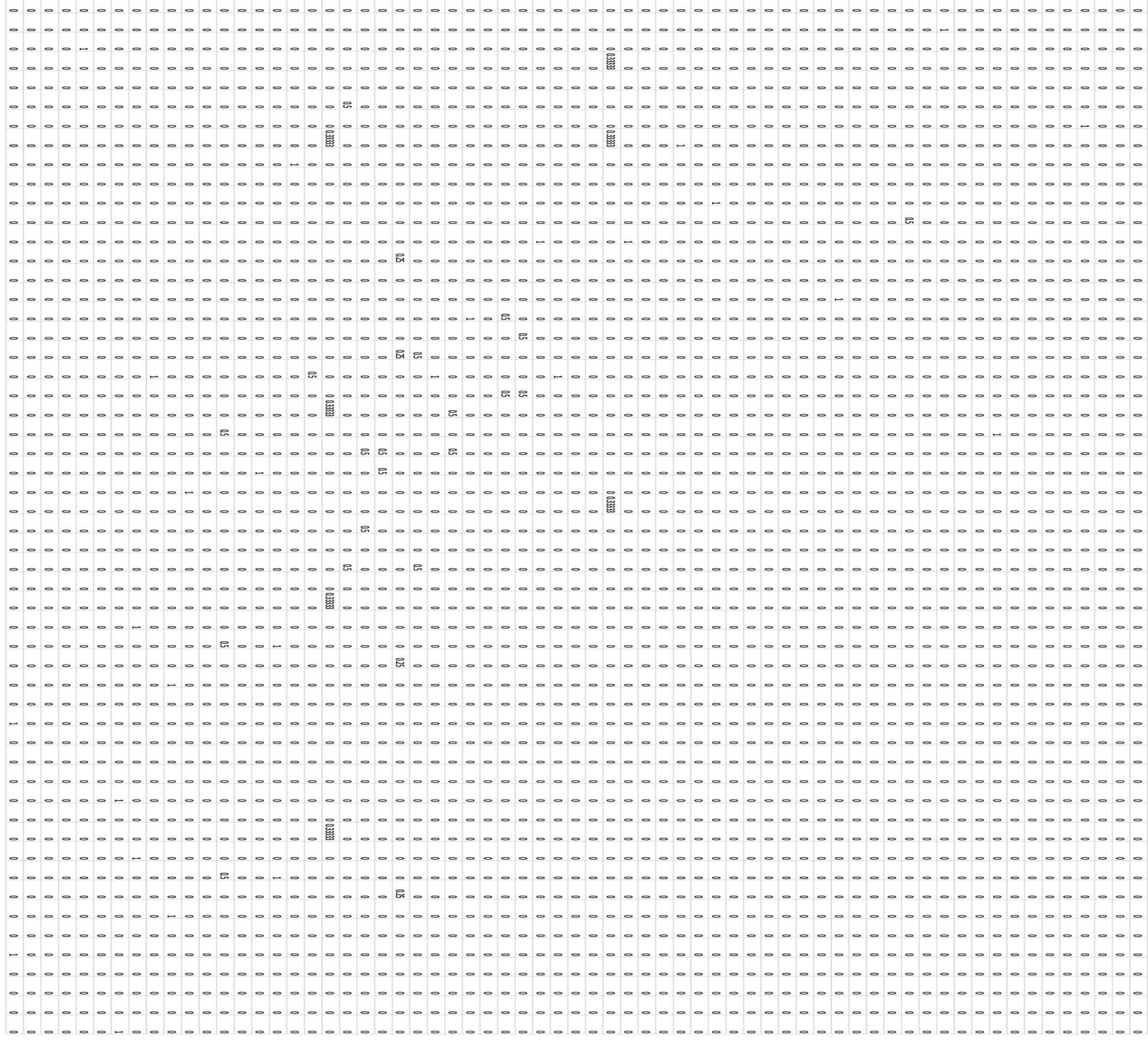


$\mathrm{P}(2)=$

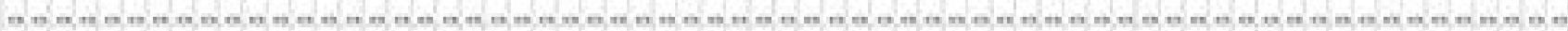

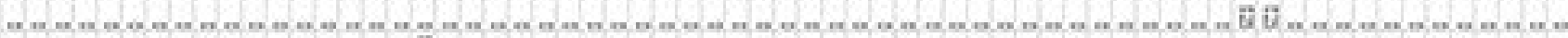

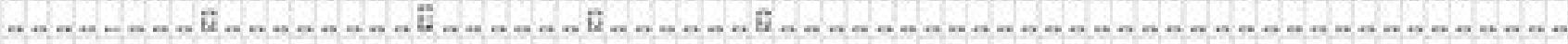

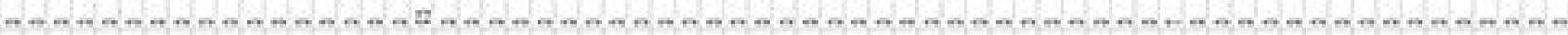

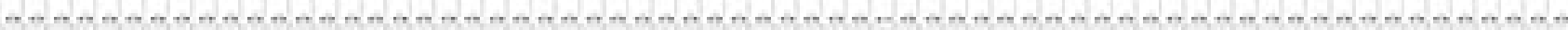

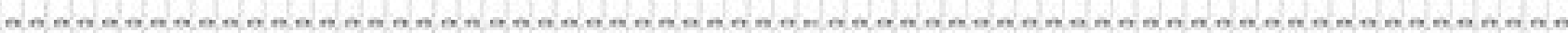

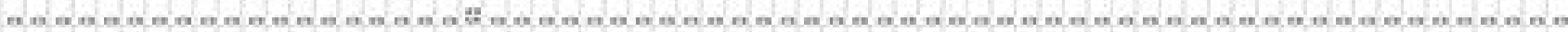
n n w w \#.

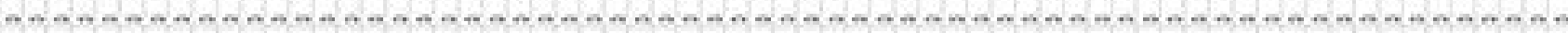

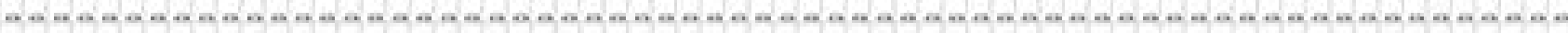

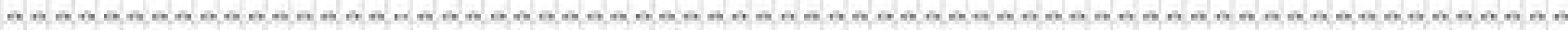

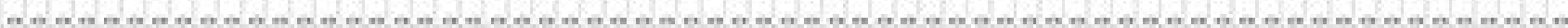

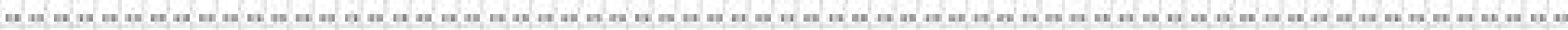

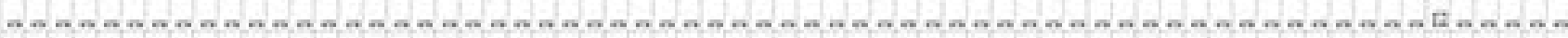

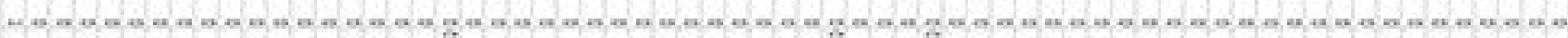

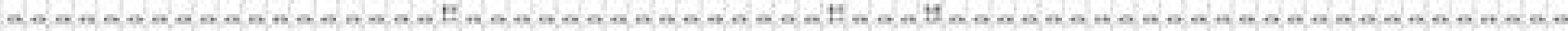

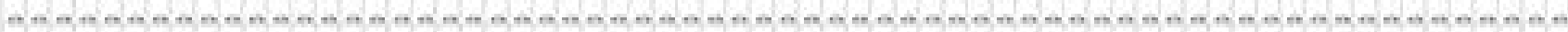

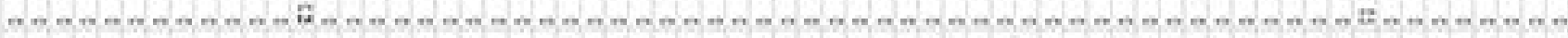

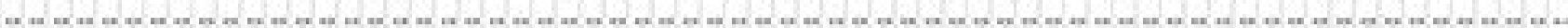

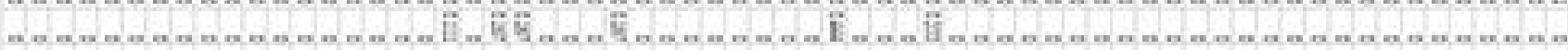

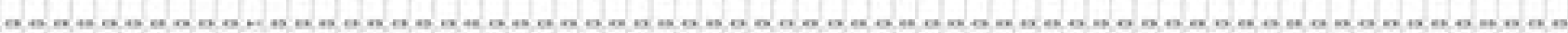

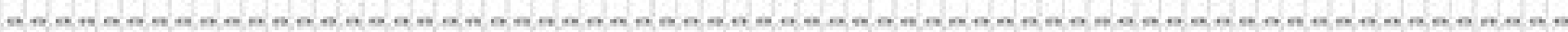

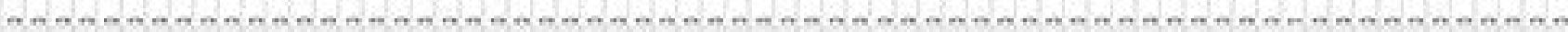

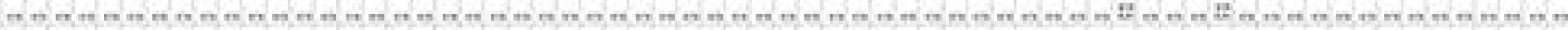

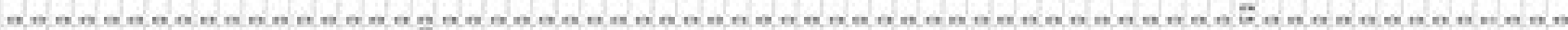

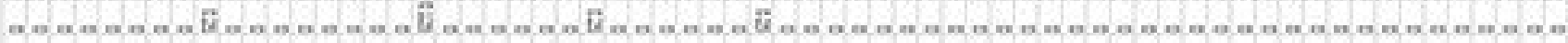

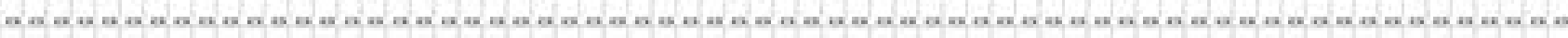

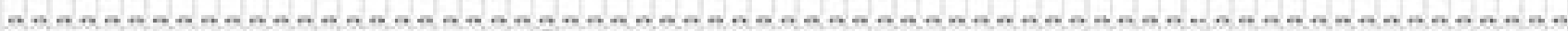

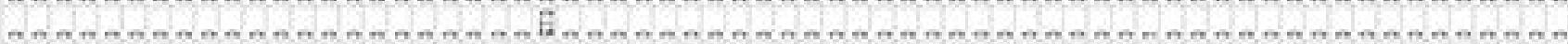

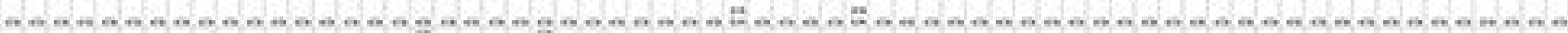

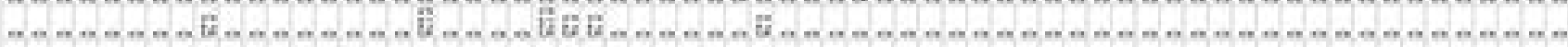

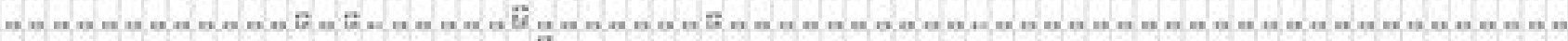

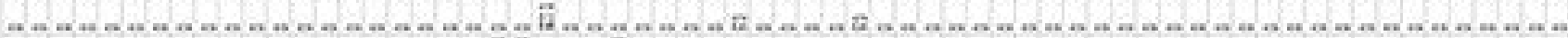
n.

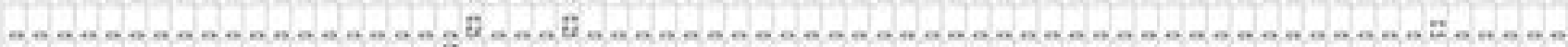

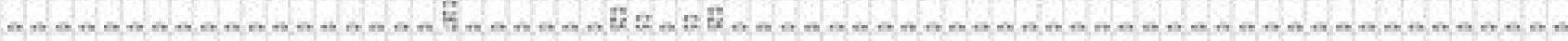

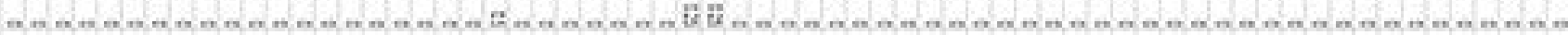

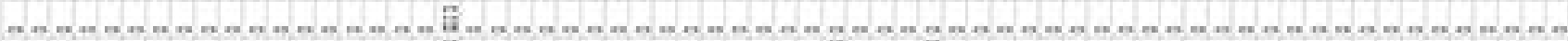

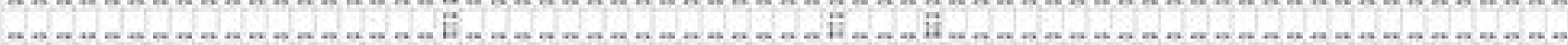

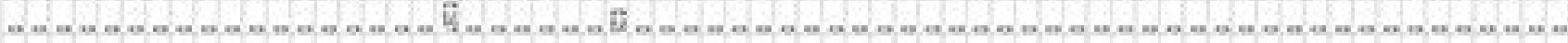

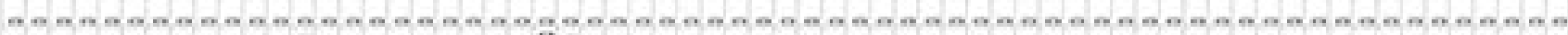

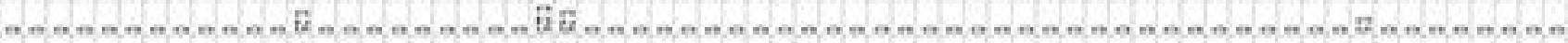

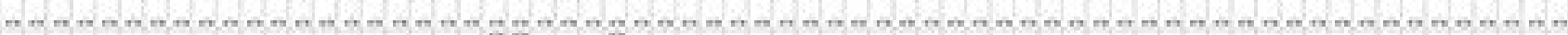
nn

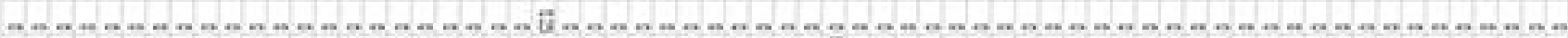

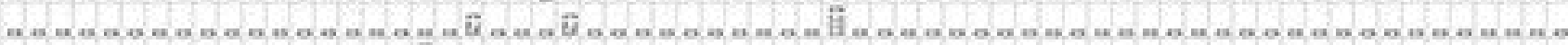

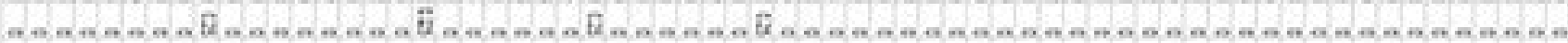

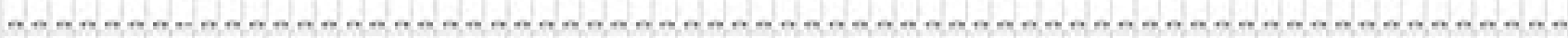

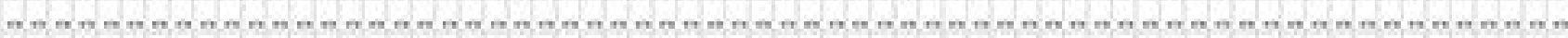

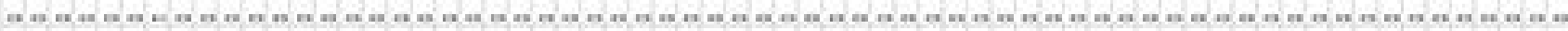

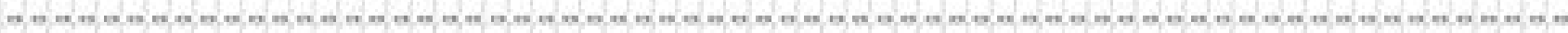

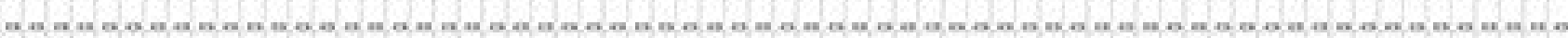

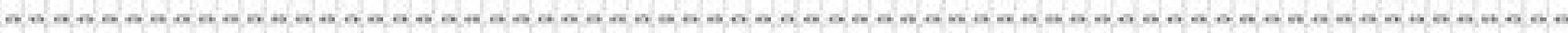

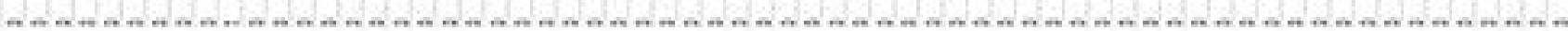


$\mathrm{P}(3)=$

0000000000000000000000 E 00000000000000000000000000000000000000000 00000000000000000000000000000000000000000000000000000000000000000 00000000000000000000000000000000000000000000000000000000000000000 00000000000000000000000000000000000000000000000000000000000000000 000000000000000000000000000000000000000000000000000000000000000

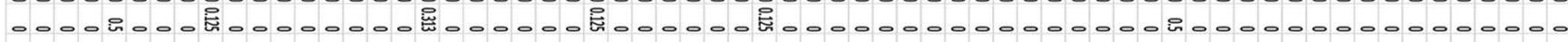
00000000000000000000000000000000000000000000000000000000000000000 00000000000000000000000000000000000000000000000000000000000000000

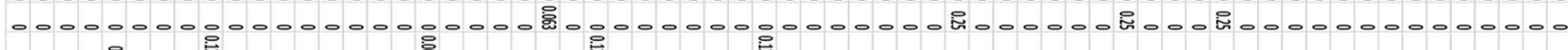

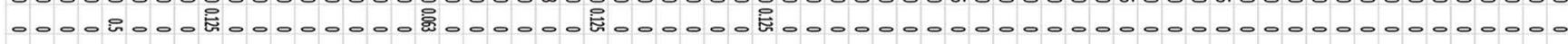
00000000000000000000000000000000000000000000000000000000000000000 00000000000000000000 每0000000000000000

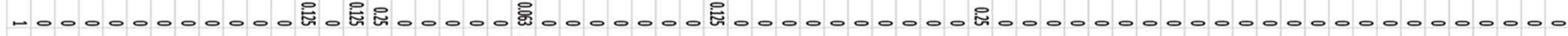

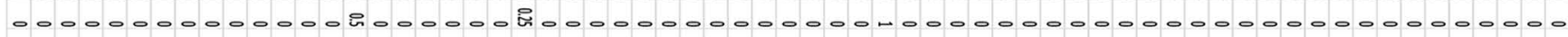
00000000000000000000000000000000000000000000000000000000000000000 00000000000000001000000000000000000000000000000000000000000000000 000000000000 니 000000000000000000000000000000000000000000000000000 000000000000000000000000000000010000000000000000000000000000000

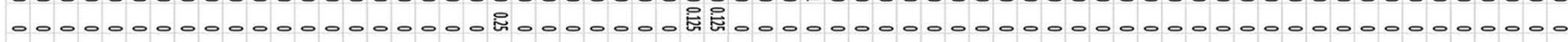
00000000000000000000000000000000000000000000000000000000000000000 00000000000000000000000000000000000000000000000000000000000000000 00000000001000000000000000000000000000000000000000000000000000000 00000000000000000000000000000000000000000000000000000000000000000 00000000000000000000000000000000000000000000000000000000000000000 $000000000000000000000000000000000000000000000000000 \mathbb{V}_{1000000000000}$ $00000-00000000000000000000000000000000000000000000000000000$ 00000000000000000000000000000000000000000000000000000000000000000

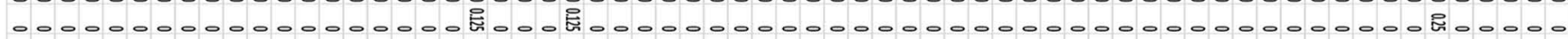

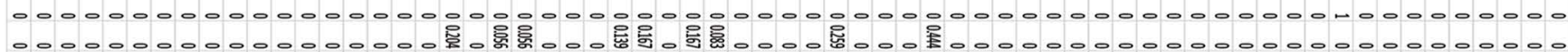
000000000000000000 ⿸\zh14⿰⿺乚一匕00000000000000000000000000000000000000000000000 00000000000000000000000000000000000000000000000000000000000000000 00000000000000000000000000000000000000000000000000000000000000000 00000000000000000 내000000000000000000000000000000 10000000000000000 00000000000000000000000000000000000000000000008000800000000000001

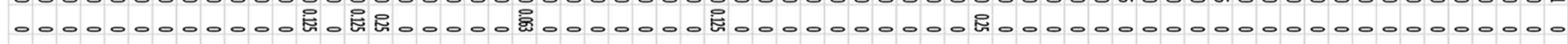
00000000000000000000000000000000000000000000000000000000000000000

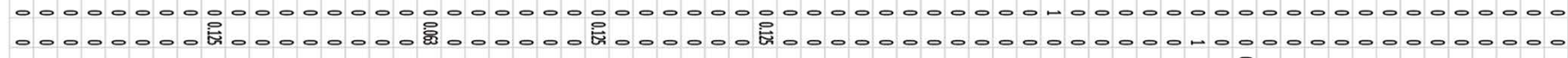

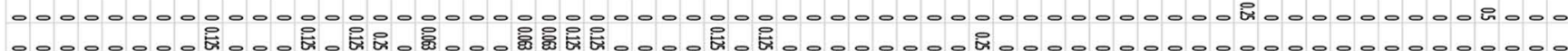

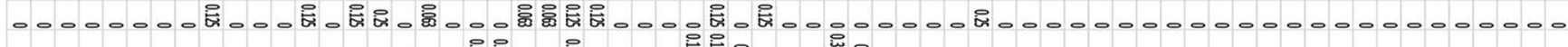

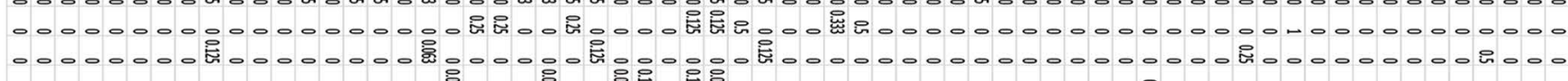
0 0 0 0 0 0

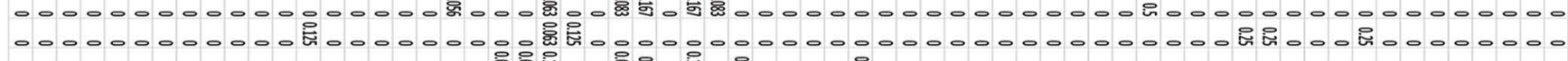

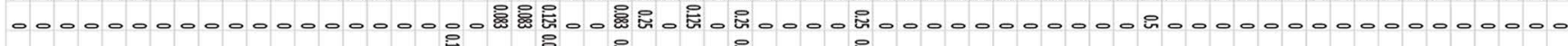

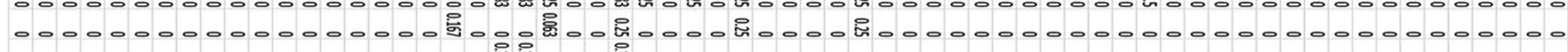

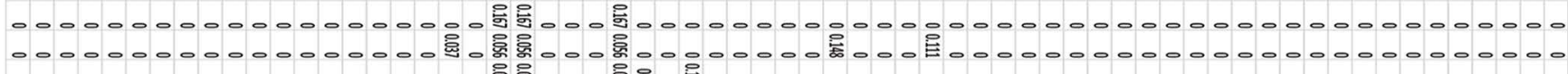

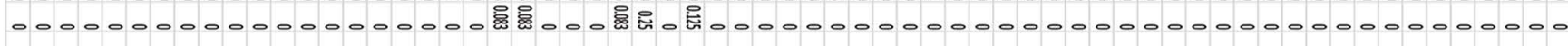
000000000000000000000000000000000000000000000000000000000000 0 0000000 000000000000000000000000000000000000000000000000000000000000000

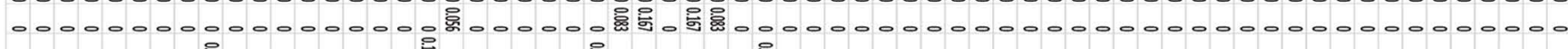

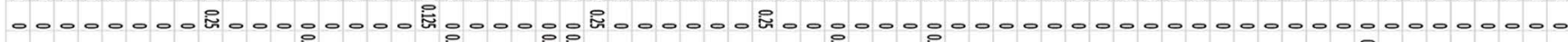

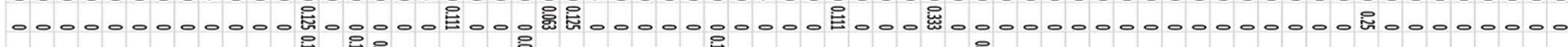

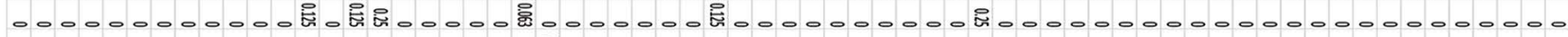

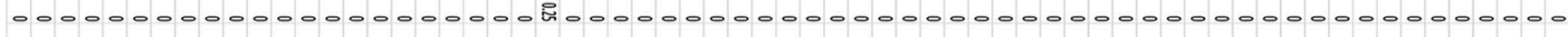
00000000000000000000000000000000000000000000000000000000000000000 00000000010000000000000000000000000000000000000000000000000000000 00000000000000000000000000000000000000000000000000000000000000000 00000000000000000000000000000000000000000000000000000000000000000 00000000000000000000000000000000000000000000000000000000000000000 00000001000000000000000000000000000000000000000000000000000000000 


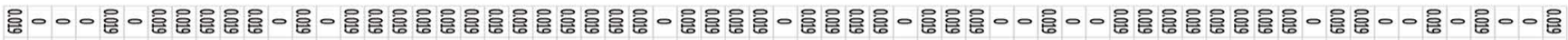
00000000000000000000000000000000000000000000000000000000000000000

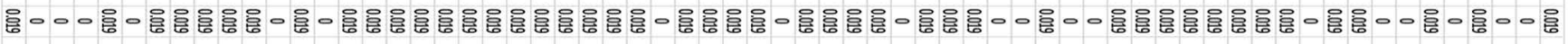
0000000000000000000000000000000000000000000000000000000000000000

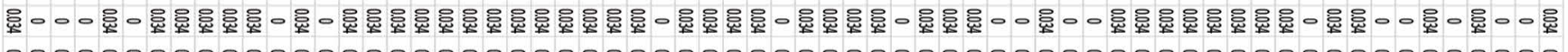
0000000000000000000000000000000000000000000000000000000000000000
00000000000000000000000000000000000000000000000000000000000000000

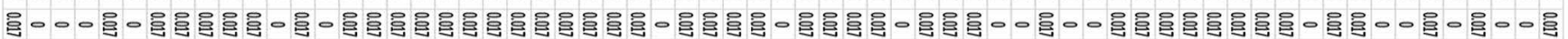

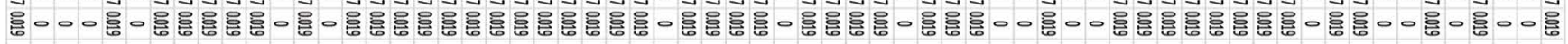

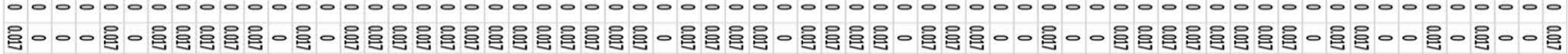

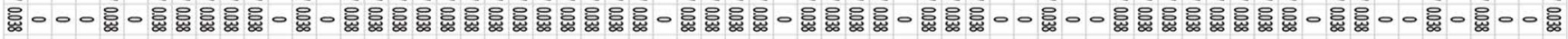
ర్ \%०00

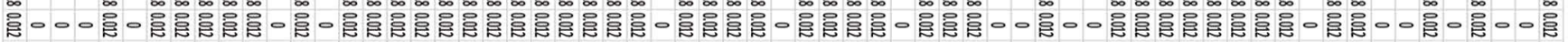

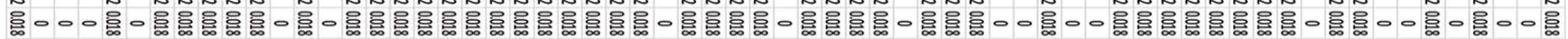

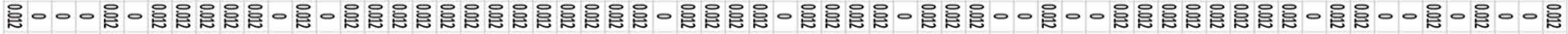

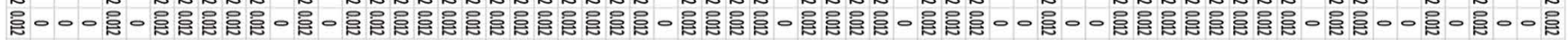
00000000000000000000000000000000000000000000000000000000000000000

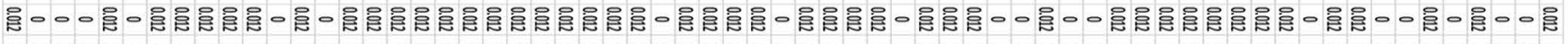
00000000000000000000000000000000000000000000000000000000000000000
00000000000000000000000000000000000000000000000000000000000000000

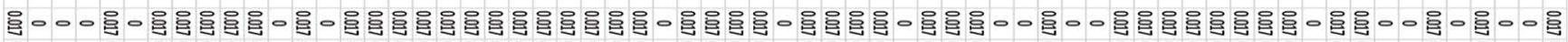

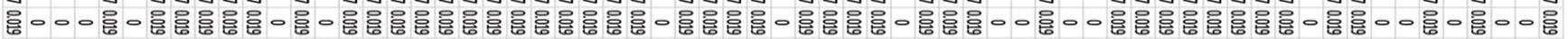

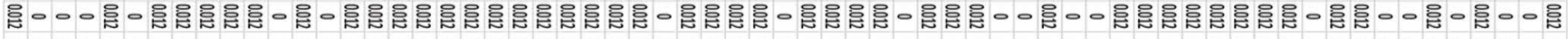
00000000000000000000000000000000000000000000000000000000000000000

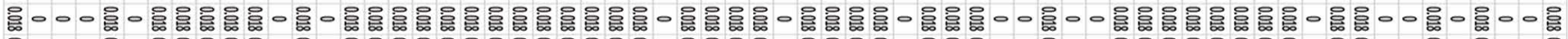
悹。00

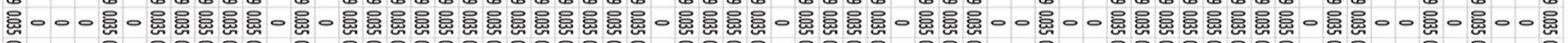

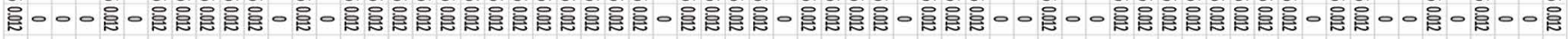
00000000000000000000000000000000000000000000000000000000000000000

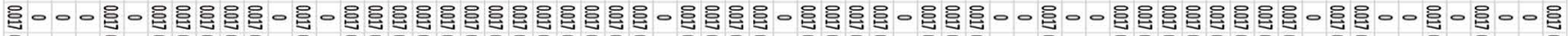

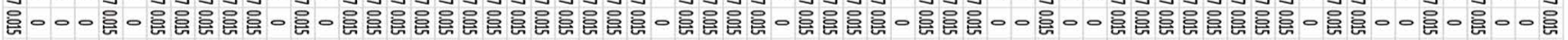

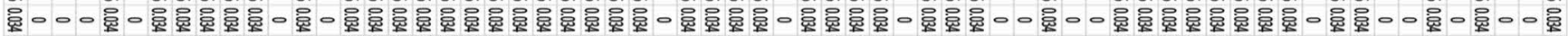

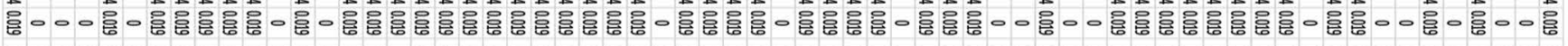

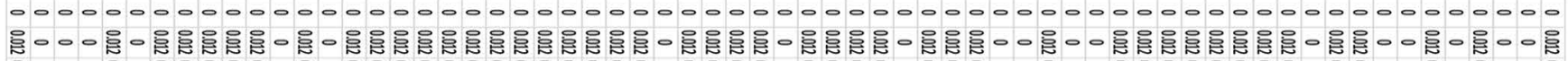

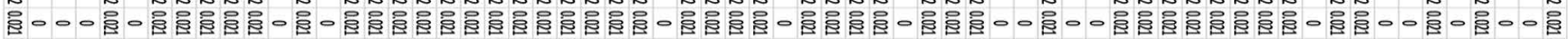

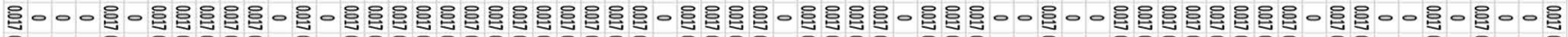

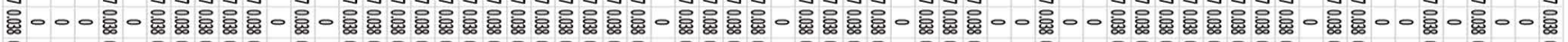

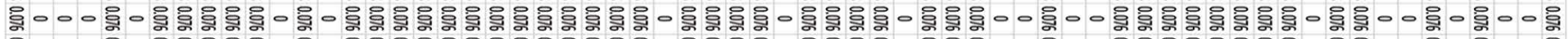

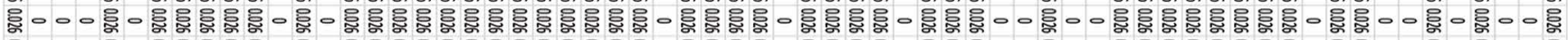

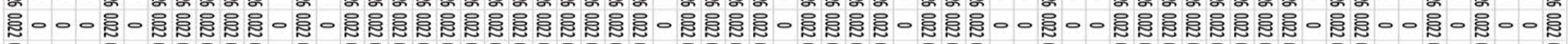

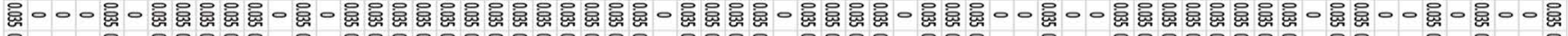

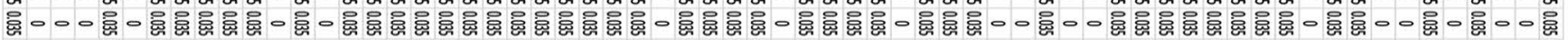

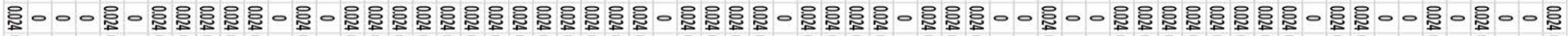

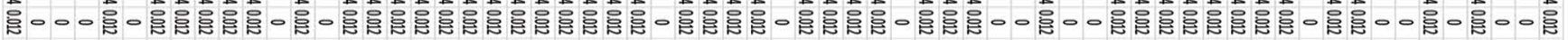

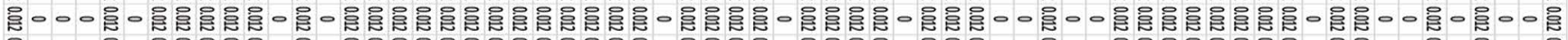

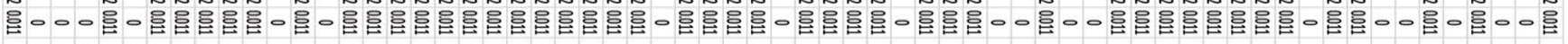
00000000000000000000000000000000000000000000000000000000000000000

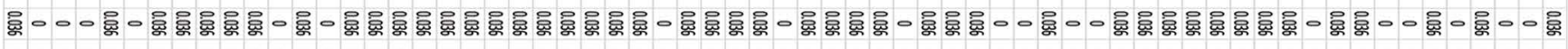
0000000000000000000000000000000000000000000000000000000000000

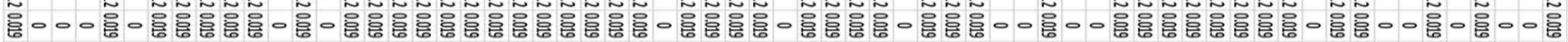
కొ00

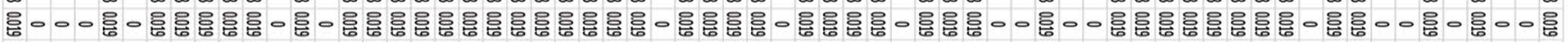

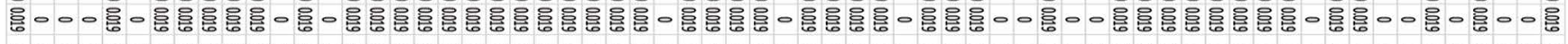
לิำ00 00000000000000000000000000000000000000000000000000000000000000000

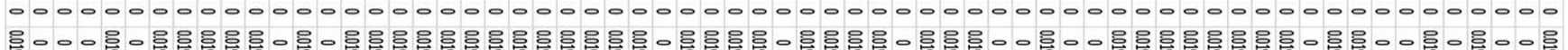




\begin{tabular}{|c|c|c|c|c|c|c|c|c|c|c|c|c|c|c|c|c|c|c|c|c|c|c|c|c|c|}
\hline & & alst & & & & & te 45 & & te 65 & & & 8 State 9 & 11 & & tate 12 & te 13 & te 14 & & & & & te 19 & & & \\
\hline \multirow{6}{*}{2018} & June & 57 & 1 & 0 & 0 & 0 & 0 & 0 & 0 & 0 & 0 & 0 & 0 & 0 & 0 & 0 & 0 & 0 & 0 & 0 & 0 & 0 & 0 & 0 & \\
\hline & May & 50 & 2 & 0 & 0 & 0 & 0 & 0 & 0 & 0 & 0 & 0 & 0 & 0 & 0 & 0 & 0 & 0 & 0 & 0 & 0 & 0 & 0 & 0 & \\
\hline & April & 31 & 3 & 0 & 0 & 0 & 0 & 0 & 0 & 0 & 0 & 0 & 0 & 0 & 0 & 0 & 0 & 0 & 0 & 0 & 0 & 0 & 0 & 0 & \\
\hline & March & 31 & 4 & 0 & 0 & 0 & 0 & 0 & 0 & 0 & 0 & 0 & 0 & 0 & 0 & 0.0833 & 0 & 0 & 0 & 0 & 0 & 0 & 0 & 0 & \\
\hline & February & 27 & 5 & 0 & 0 & 0 & 0 & 0 & 0 & 0 & 0 & 0 & 0 & & 0 & 0.0833 & 0 & 0 & 0 & 0 & 0 & 0 & 0 & 0 & \\
\hline & January & 31 & 6 & 0.042 & 0 & 0 & 0 & 0 & 0.014 & 0 & 0 & $\begin{array}{ll}0 & 0.021\end{array}$ & 0.0139 & & $\begin{array}{ll}0 & 0.0208\end{array}$ & 0.037 & 0 & 0 & 0 & 0 & 0 & 0 & 0 & 0 & \\
\hline \multirow{12}{*}{2017} & December & 47 & 7 & 0.024 & 0 & 0 & 0 & 0 & 0.005 & 0 & 0 & $\begin{array}{ll}0 & 0.033\end{array}$ & 0.0046 & & 0.033 & 0.0204 & 0.0139 & 0 & 0.0556 & 0 & 0 & 0 & o & 0 & \\
\hline & November & 40 & 8 & 0.012 & 0 & 0 & $0 \quad 0.016$ & 0 & 0.019 & 0 & 0 & $\begin{array}{ll}0 & 0.016\end{array}$ & 0.0084 & & $\begin{array}{ll}0 & 0.0165\end{array}$ & 0.0125 & 0.0069 & 0 & 0.0278 & 0 & 0.0833 & 0.0074 & 0 & 0 & 0.0139 \\
\hline & October & 39 & 9 & 0.012 & 0 & 0 & $0 \quad 0.016$ & 0 & 0.019 & 0 & 0 & $\begin{array}{ll}0 & 0.016\end{array}$ & 0.0084 & & $\begin{array}{ll}0 & 0.0165\end{array}$ & 0.0125 & 0.0069 & 0 & 0.0278 & 0 & 0.0833 & 0.0074 & 0 & 0 & 0.0139 \\
\hline & September & 18 & 10 & 0.012 & 0 & 0 & $\begin{array}{ll}0 & 0.016\end{array}$ & 0 & 0.019 & 0 & 0 & $\begin{array}{ll}0 & 0.016\end{array}$ & 0.0084 & & $\begin{array}{ll}0 & 0.0165\end{array}$ & 0.0125 & 0.0069 & 0 & 0.0278 & 0 & 0.0833 & 0.0074 & 0 & 0 & 0.0139 \\
\hline & August & 16 & 11 & 0.012 & 0 & 0 & $0 \quad 0.016$ & 0 & 0.019 & 0 & 0 & $\begin{array}{ll}0 & 0.016\end{array}$ & 0.0084 & & $\begin{array}{ll}0 & 0.0165\end{array}$ & 0.0125 & 0.0069 & 0 & 0.0278 & 0 & 0.0833 & 0.0074 & 0 & 0 & 0.0139 \\
\hline & July & 22 & 12 & 0.012 & 0 & 0 & $0 \quad 0.016$ & 0 & 0.019 & 0 & 0 & $\begin{array}{ll}0 & 0.016\end{array}$ & 0.0084 & & $\begin{array}{lll}0 & 0.0165\end{array}$ & 0.0125 & 0.0069 & 0 & 0.0278 & 0 & 0.0833 & 0.0074 & 0 & 0 & 0.0139 \\
\hline & June & 32 & 13 & 0.012 & 0 & 0 & $0 \quad 0.016$ & 0 & 0.019 & 0 & 0 & $\begin{array}{ll}0 & 0.016\end{array}$ & 0.0084 & & $\begin{array}{ll}0 & 0.0165\end{array}$ & 0.0125 & 0.0069 & 0 & 0.0278 & 0 & 0.0833 & 0.0074 & 0 & 0 & 0.0139 \\
\hline & May & 49 & 14 & 0.012 & 0 & 0 & $0 \quad 0.016$ & 0 & 0.019 & 0 & 0 & $\begin{array}{ll}0 & 0.016\end{array}$ & 0.0084 & & $\begin{array}{ll}0 & 0.0165\end{array}$ & 0.0125 & 0.0069 & 0 & 0.0278 & 0 & 0.0833 & 0.0074 & 0 & 0 & 0.0139 \\
\hline & April & 55 & 15 & 0.012 & 0 & 0 & $\begin{array}{ll}0 & 0.016\end{array}$ & 0 & 0.019 & 0 & 0 & $\begin{array}{ll}0 & 0.016\end{array}$ & 0.0084 & & $\begin{array}{ll}0 & 0.0165\end{array}$ & 0.0125 & 0.0069 & 0 & 0.0278 & 0 & 0.0833 & 0.0074 & 0 & 0 & 0.0139 \\
\hline & March & 47 & 16 & 0.014 & 0 & 0 & $\begin{array}{ll}0 & 0.022\end{array}$ & 0 & 0.028 & 0 & 0 & $\begin{array}{ll}0 & 0.015\end{array}$ & 0.0157 & & $\begin{array}{lll}0 & 0.0153\end{array}$ & 0.0282 & 0.019 & 0.0076 & 60.0233 & 0.0165 & 0.0307 & 0.0105 & 0 & 0 & 0.0147 \\
\hline & February & 45 & 17 & 0.019 & 0 & 0 & $\begin{array}{ll}0 & 0.016\end{array}$ & 0 & 0.041 & 0 & 0 & $\begin{array}{ll}0 & 0.016\end{array}$ & 0.0285 & & $\begin{array}{ll}0 & 0.0164\end{array}$ & 0.0324 & 0.0352 & 0.0147 & 0.0176 & 0.0166 & 0.0212 & 0.0085 & 0 & 0 & 0.0106 \\
\hline & January & 47 & 18 & 0.014 & 0 & 0 & $\begin{array}{ll}0 & 0.016\end{array}$ & 0 & 0.028 & 0 & 0 & $\begin{array}{ll}0 & 0.012\end{array}$ & 0.0193 & & $\begin{array}{lll}0 & 0.0118\end{array}$ & 0.0294 & 0.0244 & 0.0121 & $\begin{array}{ll}1 & 0.01\end{array}$ & 0.0209 & 0.0147 & 0.0097 & 0 & 0 & 0.0068 \\
\hline & December & 44 & 19 & 0.016 & 0 & 0 & $\begin{array}{ll}0 & 0.017\end{array}$ & 0 & 0.031 & 0 & 0 & $\begin{array}{ll}0 & 0.014\end{array}$ & 0.0173 & & $\begin{array}{lll}0 & 0.0138\end{array}$ & 0.0268 & 0.025 & 0.0121 & $1 \quad 0.0108$ & 30.0195 & 0.0107 & 0.0102 & 0 & 0 & 0.0074 \\
\hline & November & 36 & 20 & 0.016 & 0 & 0 & $\begin{array}{ll}0 & 0.022\end{array}$ & 0 & 0.028 & 0 & 0 & $0 \quad 0.017$ & 0.0152 & & $\begin{array}{ll}0 & 0.017\end{array}$ & 0.0249 & 0.0242 & 0.0127 & 0.0104 & 0.0192 & 0.0125 & 0.0115 & 0 & 0 & 0.0134 \\
\hline & October & 35 & 21 & 0.016 & 0 & 0 & $\begin{array}{ll}0 & 0.022\end{array}$ & 0 & 0.028 & 0 & 0 & $\begin{array}{ll}0 & 0.017\end{array}$ & 0.0152 & & 0.017 & 0.0249 & 0.0242 & 0.0127 & $7 \quad 0.0104$ & 0.0192 & 0.0125 & 0.0115 & & 0 & 0.0134 \\
\hline & September & 14 & 22 & 0.019 & 0 & 0 & $\begin{array}{ll}0 & 0.024\end{array}$ & 0 & 0.033 & 0 & 0 & 00.018 & 0.0164 & & $\begin{array}{ll}0 & 0.0185\end{array}$ & 0.0272 & 0.0273 & $\begin{array}{ll}3 & 0.0145\end{array}$ & 50.0108 & 30.0163 & 0.01 & 0.0102 & & 0 & 0.0117 \\
\hline & August & 15 & 23 & 0.019 & 0 & 0 & $0 \quad 0.024$ & 0 & 0.033 & 0 & 0 & 00.018 & 0.0164 & & $\begin{array}{ll}0 & 0.0185\end{array}$ & 0.0272 & 0.0273 & 0.0145 & 0.0108 & 30.0163 & 0.01 & 0.0102 & 0 & 0 & 0.0117 \\
\hline & July & 17 & 24 & 0.019 & 0 & 0 & $0 \quad 0.024$ & 0 & 0.033 & 0 & 0 & $0 \quad 0.018$ & 0.0164 & & $\begin{array}{ll}0 & 0.0185\end{array}$ & 0.0272 & 0.0273 & 30.0145 & 0.0108 & 30.0163 & 0.01 & 0.0102 & 0 & 0 & 0.0117 \\
\hline 2010 & June & 29 & 25 & 0.019 & 0 & 0 & $\begin{array}{ll}0 & 0.024\end{array}$ & 0 & 0.033 & 0 & 0 & $\begin{array}{ll}0 & 0.018\end{array}$ & 0.0164 & & $\begin{array}{ll}0 & 0.0185\end{array}$ & 0.0272 & 0.0273 & $\begin{array}{ll}3 & 0.0145\end{array}$ & 0.0108 & $\begin{array}{ll}3 & 0.0163\end{array}$ & 0.01 & 0.0102 & 0 & 0 & 0.0117 \\
\hline & May & 46 & 26 & 0.022 & 0 & 0 & $0 \quad 0.021$ & 0 & 0.034 & 0 & 0 & $\begin{array}{ll}0 & 0.019\end{array}$ & 0.018 & & $\begin{array}{ll}0 & 0.0191\end{array}$ & 0.033 & 0.0296 & 50.0157 & 7 0.0114 & 0.0165 & 0.0102 & 0.0114 & 0 & 0 & 0.0105 \\
\hline & April & 53 & 27 & 0.019 & 0 & 0 & $\begin{array}{ll}0 & 0.02\end{array}$ & 0 & 0.031 & 0 & 0 & $0 \quad 0.018$ & 0.0163 & & $\begin{array}{lll}0 & 0.0177\end{array}$ & 0.037 & 0.0287 & 0.0172 & 0.0113 & $\begin{array}{ll}3 & 0.017\end{array}$ & 0.0093 & 0.0113 & 0 & 0 & 0.0108 \\
\hline & March & 42 & 28 & 0. & 0 & 0 & 0.02 & 0 & 0.033 & 0 & 0 & 00.018 & 0.0181 & & $\begin{array}{lll}0 & 0.0178\end{array}$ & 0.0366 & 0.0296 & 0.0174 & 40.0108 & $\begin{array}{ll}3 & 0.0172\end{array}$ & 0.0104 & 0.0116 & & 0 & 0.0108 \\
\hline & February & 42 & 29 & 0.018 & 0 & 0 & 0.02 & 0 & 0.033 & 0 & 0 & $\begin{array}{ll}0 & 0.017\end{array}$ & 0.0183 & & $\begin{array}{ll}0 & 0.0167\end{array}$ & 0.0361 & 0.029 & 0.0172 & 0.0108 & 30.0182 & 0.0108 & 0.012 & & 0 & 0.0114 \\
\hline & January & 43 & 30 & 119 & 0 & 0 & $\begin{array}{ll}0 & 0.022\end{array}$ & 0 & 0.034 & 0 & 0 & $\begin{array}{ll}0 & 0.018\end{array}$ & 0.0189 & & $\begin{array}{ll}0 & 0.018\end{array}$ & 0.0339 & 0.0297 & 0.016 & $\begin{array}{ll}6 & 0.0108\end{array}$ & 0.0175 & 0.0117 & 0.0124 & & 0 & 0.0118 \\
\hline & December & 41 & 31 & 0.019 & 0 & 0 & $\begin{array}{ll}0 & 0.022\end{array}$ & 0 & 0.034 & 0 & 0 & $\begin{array}{ll}0 & 0.018\end{array}$ & 0.0189 & & 0.018 & 0.0339 & 0.0297 & 0.016 & $\begin{array}{ll}5 & 0.0108\end{array}$ & 30.0175 & 0.0117 & 0.0124 & 0 & 0 & 0.0118 \\
\hline & November & 36 & 32 & & 0 & 0 & 0.02 & 0 & 0.034 & 0 & 0 & $\begin{array}{ll}0 & 0.018\end{array}$ & 0.0189 & & $\begin{array}{ll}0 & 0.0176\end{array}$ & 0.0349 & 0.0293 & $\begin{array}{ll}3 & 0.0165\end{array}$ & 50.0107 & 0.0173 & 0.0116 & 0.0123 & 0 & 0 & 0.0114 \\
\hline & October & 30 & 33 & 0.02 & 0 & 0 & 0.02 & 0 & 0.034 & 0 & 0 & $\begin{array}{ll}0 & 0.018\end{array}$ & 0.0189 & & $\begin{array}{lll}0 & 0.0176\end{array}$ & 0.0349 & 0.0293 & 30.0165 & 0.0107 & 0.0173 & 0.0116 & 0.0123 & 0 & 0 & 0.0114 \\
\hline & September & 4 & 34 & 0.02 & 0 & 0 & 0.02 & 0 & 0.034 & 0 & 0 & $\begin{array}{ll}0 & 0.018\end{array}$ & 0.0189 & & $\begin{array}{ll}0 & 0.0176\end{array}$ & 0.0349 & 0.0293 & 30.0165 & 0.0107 & 70.0173 & 0.0116 & 0.0123 & & 0 & 0.0114 \\
\hline & August & 1 & 35 & 0.02 & 0 & 0 & 0.02 & 0 & 0.034 & 0 & 0 & $\begin{array}{ll}0 & 0.018\end{array}$ & 0.0189 & & $\begin{array}{lll}0 & 0.0176\end{array}$ & 0.0349 & 0.0293 & $\begin{array}{ll}3 & 0.0165\end{array}$ & 0.0107 & $7 \quad 0.0173$ & 0.0116 & 0.0123 & 0 & 0 & 0.0114 \\
\hline & July & 10 & 36 & 0.02 & 0 & 0 & 0.02 & 0 & 0.034 & 0 & 0 & 00.018 & 0.0189 & & $\begin{array}{lll}0 & 0.0176\end{array}$ & 0.0349 & 0.0293 & 0.0165 & $\begin{array}{ll}5 & 0.0107\end{array}$ & $7 \quad 0.0173$ & 0.0116 & 0.0123 & 0 & 0 & 0.0114 \\
\hline$\angle 013$ & June & 13 & 37 & 0.019 & 0 & 0 & 0.02 & 0 & 0.033 & 0 & 0 & $\begin{array}{ll}0 & 0.017\end{array}$ & 0.0186 & & $\begin{array}{lll}0 & 0.0169\end{array}$ & 0.0358 & 0.0291 & 0.0169 & 0.0111 & $\begin{array}{lll}1 & 0.0177\end{array}$ & 0.0116 & 0.012 & 0 & 0 & 0.0114 \\
\hline & May & 43 & 38 & 0.018 & 0 & 0 & $\begin{array}{ll}0 & 0.019\end{array}$ & 0 & 0.033 & 0 & 0 & $\begin{array}{ll}0 & 0.017\end{array}$ & 0.0187 & & $\begin{array}{ll}0 & 0.0166\end{array}$ & 0.0367 & 0.0293 & $\begin{array}{ll}3 & 0.0172\end{array}$ & 0.0116 & $\begin{array}{lll}6 & 0.0178\end{array}$ & 0.0119 & 0.0119 & & 0 & 0.0116 \\
\hline & April & 48 & 39 & 0.018 & 0 & 0 & $\begin{array}{ll}0 & 0.019\end{array}$ & 0 & 0.033 & 0 & 0 & $\begin{array}{ll}0 & 0.017\end{array}$ & 0.0186 & & $\begin{array}{ll}0 & 0.0165\end{array}$ & 0.0374 & 0.0292 & 0.0174 & 0.0116 & $\begin{array}{ll}6 \quad 0.0179\end{array}$ & 0.0118 & 0.012 & & 0 & 0.0116 \\
\hline & March & 44 & 40 & 0.019 & 0 & 0 & $\begin{array}{ll}0 & 0.019\end{array}$ & 0 & 0.033 & 0 & 0 & $\begin{array}{lll}0 & 0.017\end{array}$ & 0.0188 & & $\begin{array}{ll}0 & 0.0167\end{array}$ & 0.0378 & 0.0293 & $\begin{array}{ll}3 & 0.0176\end{array}$ & 0.0113 & $\begin{array}{ll}3 & 0.0177\end{array}$ & 0.0115 & 0.0121 & 0 & 0 & 0.0114 \\
\hline & February & 37 & 41 & 0.019 & 0 & 0 & $\begin{array}{ll}0 & 0.019\end{array}$ & 0 & 0.034 & 0 & 0 & $\begin{array}{ll}0 & 0.017\end{array}$ & 0.0189 & & $\begin{array}{ll}0 & 0.0168\end{array}$ & 0.0377 & 0.0294 & 0.0175 & 0.0112 & 20.0176 & 0.0114 & 0.0121 & 0 & 0 & 0.0114 \\
\hline & January & 43 & 4. & & 0 & 0 & $\begin{array}{ll}0 & 0.0\end{array}$ & 0 & 0.034 & 0 & 0 & $\begin{array}{lll}0 & 0.017\end{array}$ & 0.0192 & & $\begin{array}{ll}0 & 0.0171\end{array}$ & 0.0375 & 0.03 & $\begin{array}{ll}3 & 0.0175\end{array}$ & 0.0114 & $\begin{array}{ll}4 & 0.0175\end{array}$ & 0.0116 & 0.01 & & 0 & 0.0116 \\
\hline & December & 57 & 43 & 0.019 & 0 & 0 & $\begin{array}{ll}0 & 0.01\end{array}$ & 0 & 0.034 & 0 & 0 & $\begin{array}{ll}0 & 0.017\end{array}$ & 0.0192 & & $\begin{array}{ll}0 & 0.0171\end{array}$ & 0.0375 & 0.03 & 0.0175 & 0.0114 & $\begin{array}{lll}4 & 0.0175\end{array}$ & 0.0116 & 0.0121 & & 0 & 0.0116 \\
\hline & November & 53 & 44 & 0.019 & 0 & 0 & $\begin{array}{ll}0 & 0.019\end{array}$ & 0 & 0.034 & 0 & 0 & $\begin{array}{ll}0 & 0.017\end{array}$ & 0.0193 & & $\begin{array}{ll}0 & 0.0169\end{array}$ & 0.0379 & 0.03 & 0.0176 & 0.0115 & $\begin{array}{ll}5 \quad 0.0176\end{array}$ & 0.0116 & 0.01 & 0 & 0 & 0.0116 \\
\hline & October & 46 & 45 & 0.019 & 0 & 0 & $\begin{array}{ll}0 & 0.019\end{array}$ & 0 & 0.034 & 0 & 0 & $\begin{array}{ll}0 & 0.017\end{array}$ & 0.0191 & 0 & $\begin{array}{ll}0 & 0.0171\end{array}$ & 0.0376 & 0.0298 & $\begin{array}{ll}3 & 0.0175\end{array}$ & 0.0115 & $\begin{array}{ll}5 & 0.0174\end{array}$ & 0.0116 & 0.01 & & 0 & 0.0116 \\
\hline & September & 9 & 46 & 0.019 & 0 & 0 & $\begin{array}{ll}0 & 0.019\end{array}$ & 0 & 0.034 & 0 & 0 & $\begin{array}{lll}0 & 0.017\end{array}$ & 0.0191 & 0 & $\begin{array}{ll}0 & 0.0171\end{array}$ & 0.0376 & 0.0298 & $\begin{array}{ll}3 & 0.0175\end{array}$ & 0.0115 & $\begin{array}{ll}5 & 0.0174\end{array}$ & 0.0116 & 0.0121 & 0 & 0 & 0.0116 \\
\hline & August & 6 & 47 & 0.019 & 0 & 0 & $\begin{array}{ll}0 & 0.019\end{array}$ & 0 & 0.034 & 0 & 0 & $\begin{array}{ll}0 & 0.017\end{array}$ & 0.0189 & 0 & $\begin{array}{ll}0 & 0.0171\end{array}$ & 0.0376 & 0.0297 & 0.0175 & 0.0115 & $\begin{array}{lll}5 & 0.0174\end{array}$ & 0.0115 & 0.0121 & 0 & 0 & 0.0115 \\
\hline & July & 13 & 48 & 0.019 & 0 & 0 & $\begin{array}{ll}0 & 0.019\end{array}$ & 0 & 0.034 & 0 & 0 & $\begin{array}{ll}0 & 0.017\end{array}$ & 0.019 & & $\begin{array}{ll}0 & 0.017\end{array}$ & 0.038 & 0.0298 & 0.0176 & 0.0116 & $\begin{array}{ll}6 & 0.0174\end{array}$ & 0.0115 & 0.0121 & 0 & 0 & 0.0115 \\
\hline 201 & June & 26 & 49 & 0.019 & 0 & 0 & $\begin{array}{ll}0 & 0.019\end{array}$ & 0 & 0.034 & 0 & 0 & $\begin{array}{ll}0 & 0.017\end{array}$ & 0.019 & & 0.017 & 0.038 & 0.0298 & 30.0176 & 0.0116 & $\begin{array}{ll}6 & 0.0174\end{array}$ & 0.0115 & 0.0121 & 0 & 0 & 0.0115 \\
\hline & May & 61 & 50 & 0.019 & 0 & 0 & $\begin{array}{ll}0 & 0.019\end{array}$ & 0 & 0.034 & 0 & 0 & $\begin{array}{ll}0 & 0.017\end{array}$ & 0.019 & & 0.017 & 0.038 & 0.0298 & 30.0176 & 0.0116 & $\begin{array}{ll}6 & 0.0174\end{array}$ & 0.0115 & 0.0121 & 0 & 0 & 0.0115 \\
\hline & April & 65 & 51 & 0.019 & 0 & 0 & $\begin{array}{ll}0 & 0.019\end{array}$ & 0 & $\begin{array}{ll}0 & 0.034\end{array}$ & 0 & 0 & $\begin{array}{ll}0 & 0.017\end{array}$ & 0.019 & & 0.017 & 0.038 & 0.0298 & $\begin{array}{lll}8 & 0.0176\end{array}$ & 0.0116 & $\begin{array}{ll}6 & 0.0174\end{array}$ & 0.0115 & 0.0121 & 0 & 0 & 0.0115 \\
\hline & March & 59 & 52 & 0.019 & 0 & 0 & $\begin{array}{ll}0 & 0.019\end{array}$ & 0 & $\begin{array}{ll}0 & 0.034\end{array}$ & 0 & 0 & 0.017 & 0.019 & & 0.017 & 0.038 & 0.0298 & $\begin{array}{lll}8 & 0.0176\end{array}$ & 0.0116 & $\begin{array}{ll}6 & 0.0174\end{array}$ & 0.0115 & 0.0121 & 0 & 0 & 0.0115 \\
\hline & February & 56 & 5 & & 0 & 0 & $\begin{array}{ll}0 & 0.019\end{array}$ & 0 & $\begin{array}{ll}0 & 0.034\end{array}$ & 0 & 0 & 0.017 & 0.019 & & 0.017 & 0.038 & 0.0298 & $\begin{array}{ll}8 & 0.0176\end{array}$ & 0.0116 & $\begin{array}{ll}6 & 0.0174\end{array}$ & 0.0115 & 0.0121 & & 0 & 0.0115 \\
\hline & January & 58 & 54 & 0.019 & 0 & 0 & $\begin{array}{ll}0 & 0.019\end{array}$ & 0 & $\begin{array}{ll}0 & 0.034\end{array}$ & 0 & 0 & 0.017 & 0.019 & & 0.017 & 0.038 & 0.0298 & $\begin{array}{ll}8 & 0.0176\end{array}$ & 0.0116 & $\begin{array}{ll}6 & 0.0174\end{array}$ & 0.0115 & 0.0121 & & 0 & 0.0115 \\
\hline & December & 43 & 55 & 0.6 & 0 & 0 & $\begin{array}{ll}0 & 0.019\end{array}$ & 0 & $\begin{array}{ll}0 & 0.034\end{array}$ & 0 & 0 & 0.017 & 0.0189 & & $\begin{array}{ll}0 & 0.0169\end{array}$ & 0.0379 & 0.0297 & 70.0176 & 0.0116 & $\begin{array}{ll}6 \quad 0.0175 \\
\end{array}$ & 0.0116 & 0.01 & & 0 & 0.0116 \\
\hline & November & 34 & 56 & & 0 & 0 & $\begin{array}{ll}0 & 0.0\end{array}$ & 0 & $\begin{array}{ll}0 & 0.034\end{array}$ & 0 & 0 & 0.017 & 0.0189 & 0 & $\begin{array}{lll}0 & 0.0169\end{array}$ & 0.0379 & 0.0297 & $7 \quad 0.0176$ & 0.0116 & $\begin{array}{ll}6 & 0.0175\end{array}$ & 0.0116 & 0.01 & 0 & 0 & 0.0116 \\
\hline & October & 25 & 57 & & 0 & 0 & $\begin{array}{lll}0 & 0 . c\end{array}$ & 0 & $\begin{array}{ll}0 & 0.034\end{array}$ & 0 & 0 & 0.017 & 0.0189 & 0 & $\begin{array}{lll}0 & 0.0169\end{array}$ & 0.0379 & 0.0297 & $\begin{array}{ll}7 & 0.0176\end{array}$ & 0.0116 & $\begin{array}{ll}6 & 0.0175\end{array}$ & 0.0116 & 0.0121 & & 0 & 0.011 \\
\hline & September & 12 & 58 & & 0 & 0 & 0 o. & 0 & $\begin{array}{ll}0 & 0.034\end{array}$ & 0 & 0 & 0.017 & 0.0189 & 0 & $\begin{array}{ll}0 & 0.0169\end{array}$ & 0.0379 & 0.0297 & $7 \quad 0.0176$ & 0.0116 & $\begin{array}{ll}6 & 0.0175\end{array}$ & 0.0116 & 0.01 & & 0 & 0.011 \\
\hline & August & 6 & 59 & & 0 & 0 & $\begin{array}{ll}0 & 0 .\end{array}$ & 0 & $\begin{array}{ll}0 & 0.03\end{array}$ & 0 & 0 & 0. & 0.0 & 0 & $\begin{array}{lll}0 & 0.0169\end{array}$ & 0.0379 & 0.0297 & $7 \quad 0.0176$ & 0.0116 & $\begin{array}{ll}6 & 0.0175\end{array}$ & 0.0116 & 0.0121 & 0 & 0 & 0.011 \\
\hline 2013 & July & 14 & 60 & 0.019 & 0 & 0 & $\begin{array}{lll}0 & 0 .\end{array}$ & 0 & $\begin{array}{ll}0 & 0.034\end{array}$ & 0 & 0 & 0.017 & 0.0189 & 0 & $\begin{array}{ll}0 & 0.0169\end{array}$ & 0.0378 & 0.0297 & $7 \quad 0.0175$ & 0.0116 & $\begin{array}{ll}6 & 0.0175\end{array}$ & 0.0116 & 0.0121 & 0 & 0 & 0.0116 \\
\hline & June & 19 & 61 & 0.019 & 0 & 0 & $\begin{array}{ll}0 & 0.019\end{array}$ & 0 & $\begin{array}{ll}0 & 0.034\end{array}$ & 0 & 0 & 0.017 & 0.0189 & 0 & $\begin{array}{ll}0 & 0.0169\end{array}$ & 0.0378 & 0.0297 & $7 \quad 0.0175$ & 0.0116 & $\begin{array}{ll}6 & 0.0175\end{array}$ & 0.0116 & 0.0121 & 0 & 0 & 0.0116 \\
\hline & May & 48 & 62 & 0.019 & 0 & 0 & $\begin{array}{ll}0 & 0.019\end{array}$ & 0 & $\begin{array}{ll}0 & 0.034\end{array}$ & 0 & 0 & 0.017 & 0.0189 & 0 & $\begin{array}{lll}0 & 0.0169\end{array}$ & 0.0378 & 0.0297 & $\begin{array}{ll}7 & 0.0176\end{array}$ & 0.0116 & $\begin{array}{ll}6 & 0.0175\end{array}$ & 0.0116 & 0.0121 & 0 & 0 & 0.0116 \\
\hline & April & 51 & 63 & .019 & 0 & 0 & $\begin{array}{ll}0 & 0.019\end{array}$ & 0 & $\begin{array}{ll}0 & 0.034\end{array}$ & 0 & 0 & 0.017 & 0.0189 & 0 & $\begin{array}{ll}0 & 0.0169\end{array}$ & 0.0378 & 0.0297 & 70.0176 & 0.0116 & $\begin{array}{ll}6 & 0.0175\end{array}$ & 0.0116 & 0.0121 & 0 & 0 & 0.0116 \\
\hline & March & 45 & 64 & 0.019 & 0 & 0 & $\begin{array}{ll}0 & 0.019\end{array}$ & 0 & $\begin{array}{ll}0 & 0.034\end{array}$ & 0 & 0 & 0.017 & 0.0189 & & $\begin{array}{lll}0 & 0.0169\end{array}$ & 0.0378 & 0.0297 & $\begin{array}{ll}7 & 0.0176\end{array}$ & 0.0116 & $\begin{array}{ll}6 & 0.0175\end{array}$ & 0.0116 & 0.0121 & 0 & 0 & 0.0116 \\
\hline & February & 40 & 65 & 0.019 & 0 & 0 & $\begin{array}{ll}0 & 0.019\end{array}$ & 0 & $\begin{array}{ll}0 & 0.034\end{array}$ & 0 & 0 & 0.017 & 0.0189 & 0 & $\begin{array}{ll}0 & 0.0169\end{array}$ & 0.0378 & 0.0297 & 70.0176 & 0.0116 & $\begin{array}{ll}6 & 0.0175\end{array}$ & 0.0116 & 0.0121 & 0 & 0 & 0.0116 \\
\hline & & & & & & & & & & & & & & & & & & & & & & & & & \\
\hline & Sum & & & 1.1 & 0 & 0 & 1.111 & 0 & 1.837 & 0 & 0 & 1.036 & 1.0153 & & 1.0365 & 2.0782 & 1.5146 & 0.8199 & 0.8573 & 0.8756 & 1.267 & 0.6449 & & & 0.683 \\
\hline
\end{tabular}




\begin{tabular}{|c|c|c|c|c|c|c|c|c|c|c|c|c|c|c|}
\hline & & Initial State & Transition & State 23 & State 24 & State 25 & State 26 & State 27 & State 28 & State 29 & State 30 & State 31 & State 32 & State 33 \\
\hline \multirow{6}{*}{2018} & June & 57 & 1 & 0 & 0 & 0 & 0 & 0 & 0 & 0 & 0 & 0 & 0 & 0 \\
\hline & May & 50 & 2 & 0 & 0 & 0 & 0 & 0 & 0 & 0 & 0 & 0 & 0 & 0 \\
\hline & April & 31 & 3 & 0 & 0 & 0 & 0 & 0.148148148 & 0 & 0 & 0 & 0.259259259 & 0 & 0 \\
\hline & March & 31 & 4 & 0 & 0 & 0 & 0 & 0.086419753 & 0 & 0 & 0 & 0.234567901 & 0 & 0 \\
\hline & February & 27 & 5 & 0 & 0 & 0 & 0 & 0.086419753 & 0 & 0 & 0 & 0.234567901 & 0 & 0 \\
\hline & January & 31 & 6 & 0 & 0 & 0 & 0 & 0.054869684 & 0 & 0 & 0 & 0.133058985 & 0 & 0 \\
\hline \multirow{12}{*}{2017} & December & 47 & 7 & 0 & 0 & 0 & 0 & 0.024119799 & 0 & 0 & 0 & 0.063042981 & 0.009259259 & 0 \\
\hline & November & 40 & 8 & 0 & 0 & 0.0390625 & 0 & 0.021404893 & 0 & 0.00390625 & 0 & 0.054819673 & 0.00462963 & 0 \\
\hline & October & 39 & 9 & 0 & 0 & 0.0390625 & 0 & 0.021404893 & 0 & 0.00390625 & 0 & 0.054819673 & 0.00462963 & \\
\hline & September & 18 & 10 & 0 & 0 & 0.0390625 & 0 & 0.021404893 & 0 & 0.00390625 & 0 & 0.054819673 & 0.00462963 & \\
\hline & August & 16 & 11 & 0 & 0 & 0.0390625 & 0 & 0.021404893 & 0 & 0.00390625 & 0 & 0.054819673 & 0.00462963 & 0 \\
\hline & July & 22 & 12 & 0 & 0 & 0.0390625 & 0 & 0.021404893 & 0 & 0.00390625 & 0 & 0.054819673 & 0.00462963 & 0 \\
\hline & June & 32 & 13 & 0 & 0 & 0.0390625 & 0 & 0.021404893 & 0 & 0.00390625 & 0 & 0.054819673 & 0.00462963 & 0 \\
\hline & May & 49 & 14 & 0 & 0 & 0.0390625 & 0 & 0.021404893 & 0 & 0.00390625 & 0 & 0.054819673 & 0.00462963 & 0 \\
\hline & April & 55 & 15 & 0 & 0 & 0.0390625 & 0 & 0.021404893 & 0 & 0.00390625 & 0 & 0.054819673 & 0.00462963 & 0 \\
\hline & March & 47 & 16 & 0 & 0 & 0.024352809 & 0.019383597 & 0.016335013 & 0 & 0.012111432 & 0.01018137 & 0.043440266 & 0.00996216 & 0 \\
\hline & February & 45 & 17 & 0 & 0 & 0.016565033 & 0.015548913 & 0.012818906 & 0 & 0.013956111 & 0.008647301 & 0.03441059 & 0.008396161 & 0 \\
\hline & January & 47 & 18 & 0 & 0 & 0.01420594 & 0.023054173 & 0.013019445 & 0 & 0.015094076 & 0.013539393 & 0.036414614 & 0.007306299 & 0 \\
\hline \multirow{12}{*}{2016} & December & 44 & 19 & 0 & 0 & 0.018104377 & 0.018566642 & 0.011635033 & 0 & 0.018584186 & 0.02746522 & 0.034535705 & 0.012835708 & 0 \\
\hline & November & 36 & 20 & 0 & 0 & 0.018396203 & 0.026184117 & 0.011448458 & 0 & 0.019722923 & 0.021851596 & 0.033778746 & 0.011330661 & 0 \\
\hline & October & 35 & 21 & 0 & 0 & 0.018396203 & 0.026184117 & 0.011448458 & 0 & 0.019722923 & 0.021851596 & 0.033778746 & 0.011330661 & 0 \\
\hline & September & 14 & 22 & 0 & 0 & 0.020929782 & 0.018669674 & 0.011131961 & 0 & 0.019384168 & 0.024666521 & 0.032607265 & 0.012420395 & 0 \\
\hline & August & 15 & 23 & 0 & 0 & 0.020929782 & 0.018669674 & 0.011131961 & 0 & 0.019384168 & 0.024666521 & 0.032607265 & 0.012420395 & \\
\hline & July & 17 & 24 & 0 & 0 & 0.020929782 & 0.018669674 & 0.011131961 & 0 & 0.019384168 & 0.024666521 & 0.032607265 & 0.012420395 & 0 \\
\hline & June & 29 & 25 & 0 & 0 & 0.020929782 & 0.018669674 & 0.011131961 & 0 & 0.019384168 & 0.024666521 & 0.032607265 & 0.012420395 & 0 \\
\hline & May & 46 & 26 & 0 & 0 & 0.018387356 & 0.016861451 & 0.010885712 & 0 & 0.018300906 & 0.022755295 & 0.033024057 & 0.011853813 & 0 \\
\hline & April & 53 & 27 & 0 & 0 & 0.017944622 & 0.017861142 & 0.011229579 & 0 & 0.019135275 & 0.024198255 & 0.0337857 & 0.011905443 & 0 \\
\hline & March & 42 & 28 & 0 & 0 & 0.017966092 & 0.017957564 & 0.011069178 & 0 & 0.018178815 & 0.021133839 & 0.033496616 & 0.011353985 & 0 \\
\hline & February & 42 & 29 & 0 & 0 & 0.017826432 & 0.019687421 & 0.011165539 & 0 & 0.018191064 & 0.02039872 & 0.033754373 & 0.011628155 & 0 \\
\hline & January & 43 & 30 & 0 & 0 & 0.018321412 & 0.019005764 & 0.011453993 & 0 & 0.017252956 & 0.018420223 & 0.034346825 & 0.011428734 & 0 \\
\hline \multirow{12}{*}{2015} & December & 41 & 31 & 0 & 0 & 0.018321412 & 0.019005764 & 0.011453993 & 0 & 0.017252956 & 0.018420223 & 0.034346825 & 0.011428734 & 0 \\
\hline & November & 36 & 32 & 0 & 0 & 0.017528823 & 0.018649013 & 0.011635759 & 0 & 0.017379964 & 0.019029379 & 0.034974827 & 0.011736144 & 0 \\
\hline & October & 30 & 33 & 0 & 0 & 0.017528823 & 0.018649013 & 0.011635759 & 0 & 0.017379964 & 0.019029379 & 0.034974827 & 0.011736144 & 0 \\
\hline & September & 4 & 34 & 0 & 0 & 0.017528823 & 0.018649013 & 0.011635759 & 0 & 0.017379964 & 0.019029379 & 0.034974827 & 0.011736144 & 0 \\
\hline & August & 1 & 35 & 0 & 0 & 0.017528823 & 0.018649013 & 0.011635759 & 0 & 0.017379964 & 0.019029379 & 0.034974827 & 0.011736144 & 0 \\
\hline & July & 10 & 36 & 0 & 0 & 0.017528823 & 0.018649013 & 0.011635759 & 0 & 0.017379964 & 0.019029379 & 0.034974827 & 0.011736144 & 0 \\
\hline & June & 13 & 37 & 0 & 0 & 0.017374278 & 0.019365869 & 0.011547728 & 0 & 0.017809361 & 0.019575214 & 0.034615818 & 0.011610215 & 0 \\
\hline & May & 43 & 38 & 0 & 0 & 0.017206942 & 0.019584416 & 0.011590586 & 0 & 0.01778995 & 0.019383712 & 0.034577041 & 0.011613679 & 0 \\
\hline & April & 48 & 39 & 0 & 0 & 0.016920493 & 0.019717508 & 0.01156129 & 0 & 0.017873251 & 0.01945366 & 0.034583902 & 0.011621863 & 0 \\
\hline & March & 44 & 40 & 0 & 0 & 0.016654959 & 0.019242115 & 0.01159974 & 0 & 0.017666434 & 0.019101901 & 0.03486078 & 0.011622738 & 0 \\
\hline & February & 37 & 41 & 0 & 0 & 0.016706213 & 0.019026511 & 0.011602838 & 0 & 0.017567013 & 0.018942598 & 0.034915341 & 0.011573943 & 0 \\
\hline & January & 43 & 42 & 0 & 0 & 0.016947121 & 0.018777184 & 0.011548201 & 0 & 0.017322316 & 0.018326655 & 0.034677267 & 0.011444158 & 0 \\
\hline \multirow{12}{*}{2014} & December & 57 & 43 & 0 & 0 & 0.016947121 & 0.018777184 & 0.011548201 & 0 & 0.017322316 & 0.018326655 & 0.034677267 & 0.011444158 & 0 \\
\hline & November & 53 & 44 & 0 & 0 & 0.016799628 & 0.018907871 & 0.01154865 & 0 & 0.017405336 & 0.018386361 & 0.03463222 & 0.011475218 & 0 \\
\hline & October & 46 & 45 & 0 & 0 & 0.016991411 & 0.018692595 & 0.011547132 & 0 & 0.017444444 & 0.018728256 & 0.034668241 & 0.011505725 & 0 \\
\hline & September & 9 & 46 & 0 & 0 & 0.016991411 & 0.018692595 & 0.011547132 & 0 & 0.017444444 & 0.018728256 & 0.034668241 & 0.011505725 & 0 \\
\hline & August & 6 & 47 & 0 & 0 & 0.01702475 & 0.018557223 & 0.01155311 & 0 & 0.017509563 & 0.01902739 & 0.03470656 & 0.011540996 & 0 \\
\hline & July & 13 & 48 & 0 & 0 & 0.016812812 & 0.018638119 & 0.011565341 & 0 & 0.017485626 & 0.01886924 & 0.03473542 & 0.011581306 & 0 \\
\hline & June & 26 & 49 & 0 & 0 & 0.016812812 & 0.018638119 & 0.011565341 & 0 & 0.017485626 & 0.01886924 & 0.03473542 & 0.011581306 & 0 \\
\hline & May & 61 & 50 & 0 & 0 & 0.016812812 & 0.018638119 & 0.011565341 & 0 & 0.017485626 & 0.01886924 & 0.03473542 & 0.011581306 & 0 \\
\hline & April & 65 & 51 & 0 & 0 & 0.016812812 & 0.018638119 & 0.011565341 & 0 & 0.017485626 & 0.01886924 & 0.03473542 & 0.011581306 & 0 \\
\hline & March & 59 & 52 & 0 & 0 & 0.016812812 & 0.018638119 & 0.011565341 & 0 & 0.017485626 & 0.01886924 & 0.03473542 & 0.011581306 & 0 \\
\hline & February & 56 & 53 & 0 & 0 & 0.016812812 & 0.018638119 & 0.011565341 & 0 & 0.017485626 & 0.01886924 & 0.03473542 & 0.011581306 & 0 \\
\hline & January & 58 & 54 & 0 & 0 & 0.016812812 & 0.018638119 & 0.011565341 & 0 & 0.017485626 & 0.01886924 & 0.03473542 & 0.011581306 & 0 \\
\hline \multirow{11}{*}{2013} & December & 43 & 55 & 0 & 0 & 0.016846995 & 0.018811519 & 0.011572256 & 0 & 0.017525115 & 0.018896672 & 0.034726908 & 0.01157864 & 0 \\
\hline & November & 34 & 56 & 0 & 0 & 0.016846995 & 0.018811519 & 0.011572256 & 0 & 0.017525115 & 0.018896672 & 0.034726908 & 0.01157864 & 0 \\
\hline & October & 25 & 57 & 0 & 0 & 0.016846995 & 0.018811519 & 0.011572256 & 0 & 0.017525115 & 0.018896672 & 0.034726908 & 0.01157864 & 0 \\
\hline & September & 12 & 58 & 0 & 0 & 0.016846995 & 0.018811519 & 0.011572256 & 0 & 0.017525115 & 0.018896672 & 0.034726908 & 0.01157864 & 0 \\
\hline & August & 6 & 59 & 0 & 0 & 0.016843365 & 0.018853779 & 0.011571387 & 0 & 0.01752065 & 0.018850538 & 0.034720365 & 0.011571974 & 0 \\
\hline & July & 14 & 60 & 0 & 0 & 0.016870149 & 0.018865858 & 0.011572263 & 0 & 0.017528666 & 0.018878237 & 0.034718369 & 0.011569096 & 0 \\
\hline & June & 19 & 61 & 0 & 0 & 0.016870149 & 0.018865858 & 0.011572263 & 0 & 0.017528666 & 0.018878237 & 0.034718369 & 0.011569096 & 0 \\
\hline & May & 48 & 62 & 0 & 0 & 0.016857021 & 0.018873153 & 0.011571548 & 0 & 0.017533637 & 0.018883636 & 0.034718503 & 0.01156976 & 0 \\
\hline & April & 51 & 63 & 0 & 0 & 0.016857021 & 0.018873153 & 0.011571548 & 0 & 0.017533637 & 0.018883636 & 0.034718503 & 0.01156976 & 0 \\
\hline & March & 45 & 64 & 0 & 0 & 0.016863263 & 0.01889393 & 0.011573702 & 0 & 0.017543937 & 0.018904942 & 0.034719844 & 0.011573868 & 0 \\
\hline & February & 40 & 65 & 0 & 0 & 0.016866543 & 0.018908379 & 0.011573715 & 0 & 0.017546915 & 0.018903365 & 0.03471709 & 0.01157005 & 0 \\
\hline & & & & & & & & & & & & & & \\
\hline & Sum & & & & 0 & 1.192320636 & 0.954412517 & 1.15333367 & & 0.911580489 & 0.970510624 & 3.094139967 & 0.620754835 & \\
\hline
\end{tabular}




\begin{tabular}{|c|c|c|c|c|c|c|c|c|c|c|c|c|c|c|}
\hline & & Initial State & Transition & State 34 & State 35 & State 36 & State 37 & State 38 & State 39 & State 40 & State 41 & State 42 & State 43 & State 44 \\
\hline \multirow{6}{*}{2018} & June & 57 & 1 & 0 & 0 & 0 & 0 & 0 & 0 & 0 & 0 & 0 & 1 & 0 \\
\hline & May & 50 & 2 & 0 & 0 & 0 & 0 & 0 & 0 & 0 & 0 & 0 & 1 & 0 \\
\hline & April & 31 & 3 & 0 & 0 & 0 & 0 & 0 & 0 & 0 & 0 & 0 & 0.333333333 & 0 \\
\hline & March & 31 & 4 & 0 & 0 & 0 & 0.083333333 & 0 & 0 & 0 & 0 & 0.083333333 & 0.111111111 & 0 \\
\hline & February & 27 & 5 & 0 & 0 & 0 & 0.083333333 & 0 & 0 & 0 & 0 & 0.083333333 & 0.111111111 & 0 \\
\hline & January & 31 & 6 & 0 & 0 & 0 & 0.037037037 & 0 & 0 & 0.013888889 & 0 & 0.071759259 & 0.086419753 & 0.013888889 \\
\hline \multirow{12}{*}{2017} & December & 47 & 7 & 0 & 0 & 0.041666667 & 0.020447531 & 0 & 0.333333333 & 0.00462963 & 0 & 0.037229938 & 0.034979424 & 0.00462963 \\
\hline & November & 40 & 8 & 0 & 0.010416667 & 0.020833333 & 0.01253858 & 0 & 0.166666667 & 0.008391204 & 0.03125 & 0.030912423 & 0.049655993 & 0.039641204 \\
\hline & October & 39 & 9 & 0 & 0.010416667 & 0.020833333 & 0.01253858 & 0 & 0.166666667 & 0.008391204 & 0.03125 & 0.030912423 & 0.049655993 & 0.039641204 \\
\hline & September & 18 & 10 & 0 & 0.010416667 & 0.020833333 & 0.01253858 & 0 & 0.166666667 & 0.008391204 & 0.03125 & 0.030912423 & 0.049655993 & 0.039641204 \\
\hline & August & 16 & 11 & 0 & 0.010416667 & 0.020833333 & 0.01253858 & 0 & 0.166666667 & 0.008391204 & 0.03125 & 0.030912423 & 0.049655993 & 0.039641204 \\
\hline & July & 22 & 12 & 0 & 0.010416667 & 0.020833333 & 0.01253858 & 0 & 0.166666667 & 0.008391204 & 0.03125 & 0.030912423 & 0.049655993 & 0.039641204 \\
\hline & June & 32 & 13 & 0 & 0.010416667 & 0.020833333 & 0.01253858 & 0 & 0.166666667 & 0.008391204 & 0.03125 & 0.030912423 & 0.049655993 & 0.039641204 \\
\hline & May & 49 & 14 & 0 & 0.010416667 & 0.020833333 & 0.01253858 & 0 & 0.166666667 & 0.008391204 & 0.03125 & 0.030912423 & 0.049655993 & 0.039641204 \\
\hline & April & 55 & 15 & 0 & 0.010416667 & 0.020833333 & 0.01253858 & 0 & 0.166666667 & 0.008391204 & 0.03125 & 0.030912423 & 0.049655993 & 0.039641204 \\
\hline & March & 47 & 16 & 0.011187168 & 0.012751306 & 0.051104892 & 0.014579538 & 0 & 0.064171811 & 0.012245954 & 0.016883742 & 0.032197481 & 0.07704041 & 0.025303821 \\
\hline & February & 45 & 17 & 0.012587007 & 0.012874625 & 0.033589872 & 0.018203793 & 0 & 0.035087931 & 0.014068934 & 0.01190053 & 0.037976087 & 0.08487173 & 0.023622499 \\
\hline & January & 47 & 18 & 0.015323465 & 0.009076332 & 0.031231314 & 0.018306848 & 0 & 0.023311132 & 0.040354931 & 0.011466338 & 0.03598627 & 0.090757632 & 0.021096389 \\
\hline \multirow{12}{*}{2016} & December & 44 & 19 & 0.02125898 & 0.013898164 & 0.034986876 & 0.01599118 & 0 & 0.014959 & 0.030190036 & 0.01493604 & 0.034522288 & 0.077149075 & 0.023810824 \\
\hline & November & 36 & 20 & 0.021890755 & 0.013160239 & 0.045375532 & 0.014545882 & 0 & 0.011283522 & 0.021788194 & 0.012447469 & 0.033068734 & 0.082461391 & 0.020768913 \\
\hline & October & 35 & 21 & 0.021890755 & 0.013160239 & 0.045375532 & 0.014545882 & 0 & 0.011283522 & 0.021788194 & 0.012447469 & 0.033068734 & 0.082461391 & 0.020768913 \\
\hline & September & 14 & 22 & 0.020831116 & 0.016488891 & 0.035967776 & 0.015408461 & 0 & 0.010272898 & 0.01983709 & 0.014482438 & 0.035375092 & 0.069629354 & 0.023647501 \\
\hline & August & 15 & 23 & 0.020831116 & 0.016488891 & 0.035967776 & 0.015408461 & 0 & 0.010272898 & 0.01983709 & 0.014482438 & 0.035375092 & 0.069629354 & 0.023647501 \\
\hline & July & 17 & 24 & 0.020831116 & 0.016488891 & 0.035967776 & 0.015408461 & 0 & 0.010272898 & 0.01983709 & 0.014482438 & 0.035375092 & 0.069629354 & 0.023647501 \\
\hline & June & 29 & 25 & 0.020831116 & 0.016488891 & 0.035967776 & 0.015408461 & 0 & 0.010272898 & 0.01983709 & 0.014482438 & 0.035375092 & 0.069629354 & 0.023647501 \\
\hline & May & 46 & 26 & 0.018926547 & 0.016357268 & 0.03268505 & 0.017710207 & 0 & 0.011747405 & 0.019205098 & 0.014922386 & 0.037561693 & 0.069910274 & 0.024451232 \\
\hline & April & 53 & 27 & 0.019793558 & 0.014718707 & 0.033448565 & 0.018357986 & 0 & 0.011890043 & 0.019211858 & 0.018534856 & 0.037068958 & 0.070914362 & 0.027308756 \\
\hline & March & 42 & 28 & 0.017987563 & 0.015236945 & 0.033824605 & 0.018534479 & 0 & 0.011507851 & 0.020481146 & 0.017100323 & 0.037694823 & 0.07252614 & 0.026455809 \\
\hline & February & 42 & 29 & 0.017966092 & 0.014798236 & 0.036370783 & 0.018131535 & 0 & 0.010407437 & 0.020775091 & 0.016912303 & 0.036978946 & 0.076398515 & 0.026179542 \\
\hline & January & 43 & 30 & 0.016686181 & 0.014981119 & 0.037034941 & 0.017845372 & 0 & 0.012736357 & 0.021613976 & 0.014800375 & 0.037302669 & 0.074881114 & 0.024457276 \\
\hline \multirow{12}{*}{2015} & December & 41 & 31 & 0.016686181 & 0.014981119 & 0.037034941 & 0.017845372 & 0 & 0.012736357 & 0.021613976 & 0.014800375 & 0.037302669 & 0.074881114 & 0.024457276 \\
\hline & November & 36 & 32 & 0.016869785 & 0.015123092 & 0.035277444 & 0.018072236 & 0 & 0.011623381 & 0.021345441 & 0.015197399 & 0.037404079 & 0.074510636 & 0.024803823 \\
\hline & October & 30 & 33 & 0.016869785 & 0.015123092 & 0.035277444 & 0.018072236 & 0 & 0.011623381 & 0.021345441 & 0.015197399 & 0.037404079 & 0.074510636 & 0.024803823 \\
\hline & September & 4 & 34 & 0.016869785 & 0.015123092 & 0.035277444 & 0.018072236 & 0 & 0.011623381 & 0.021345441 & 0.015197399 & 0.037404079 & 0.074510636 & 0.024803823 \\
\hline & August & 1 & 35 & 0.016869785 & 0.015123092 & 0.035277444 & 0.018072236 & 0 & 0.011623381 & 0.021345441 & 0.015197399 & 0.037404079 & 0.074510636 & 0.024803823 \\
\hline & July & 10 & 36 & 0.016869785 & 0.015123092 & 0.035277444 & 0.018072236 & 0 & 0.011623381 & 0.021345441 & 0.015197399 & 0.037404079 & 0.074510636 & 0.024803823 \\
\hline & June & 13 & 37 & 0.017466922 & 0.014741357 & 0.035441145 & 0.018232965 & 0 & 0.011355031 & 0.021277755 & 0.016235552 & 0.037229332 & 0.075596975 & 0.025652006 \\
\hline & May & 43 & 38 & 0.017381843 & 0.014702381 & 0.035454557 & 0.018421852 & 0 & 0.011320706 & 0.020855305 & 0.016611856 & 0.037269369 & 0.076274527 & 0.026000448 \\
\hline & April & 48 & 39 & 0.017463093 & 0.014603192 & 0.035107869 & 0.018628115 & 0 & 0.011281782 & 0.020724886 & 0.016998901 & 0.03736291 & 0.076550052 & 0.026312527 \\
\hline & March & 44 & 40 & 0.017080886 & 0.014674994 & 0.034130585 & 0.01881967 & 0 & 0.011365473 & 0.020995809 & 0.016897027 & 0.037643926 & 0.076051834 & 0.026284236 \\
\hline & February & 37 & 41 & 0.016911714 & 0.014713972 & 0.033848268 & 0.018846263 & 0 & 0.011472655 & 0.021197951 & 0.016752722 & 0.037742213 & 0.07573356 & 0.02619085 \\
\hline & January & 43 & 42 & 0.016477678 & 0.014912758 & 0.033734227 & 0.018903385 & 0 & 0.011613679 & 0.021231099 & 0.016402967 & 0.037981078 & 0.075554788 & 0.026012855 \\
\hline \multirow{12}{*}{2014} & December & 57 & 43 & 0.016477678 & 0.014912758 & 0.033734227 & 0.018903385 & 0 & 0.011613679 & 0.021231099 & 0.016402967 & 0.037981078 & 0.075554788 & 0.026012855 \\
\hline & November & 53 & 44 & 0.01658109 & 0.014821818 & 0.033647745 & 0.018983528 & 0 & 0.011581785 & 0.021201485 & 0.016701689 & 0.0379705 & 0.075904802 & 0.026267509 \\
\hline & October & 46 & 45 & 0.016696584 & 0.014936829 & 0.033587713 & 0.018899097 & 0 & 0.011605916 & 0.021152501 & 0.016588472 & 0.03792093 & 0.075292366 & 0.026120324 \\
\hline & September & 9 & 46 & 0.016696584 & 0.014936829 & 0.033587713 & 0.018899097 & 0 & 0.011605916 & 0.021152501 & 0.016588472 & 0.03792093 & 0.075292366 & 0.026120324 \\
\hline & August & 6 & 47 & 0.016821852 & 0.014962555 & 0.033413563 & 0.018893897 & 0 & 0.011617383 & 0.021108936 & 0.016727793 & 0.037900083 & 0.074965143 & 0.026219565 \\
\hline & July & 13 & 48 & 0.016758229 & 0.01493372 & 0.033210184 & 0.019007118 & 0 & 0.011544242 & 0.021068612 & 0.016826559 & 0.037973478 & 0.075321445 & 0.02632651 \\
\hline & June & 26 & 49 & 0.016758229 & 0.01493372 & 0.033210184 & 0.019007118 & 0 & 0.011544242 & 0.021068612 & 0.016826559 & 0.037973478 & 0.075321445 & 0.02632651 \\
\hline & May & 61 & 50 & 0.016758229 & 0.01493372 & 0.033210184 & 0.019007118 & 0 & 0.011544242 & 0.021068612 & 0.016826559 & 0.037973478 & 0.075321445 & 0.02632651 \\
\hline & April & 65 & 51 & 0.016758229 & 0.01493372 & 0.033210184 & 0.019007118 & 0 & 0.011544242 & 0.021068612 & 0.016826559 & 0.037973478 & 0.075321445 & 0.02632651 \\
\hline & March & 59 & 52 & 0.016758229 & 0.01493372 & 0.033210184 & 0.019007118 & 0 & 0.011544242 & 0.021068612 & 0.016826559 & 0.037973478 & 0.075321445 & 0.02632651 \\
\hline & February & 56 & 53 & 0.016758229 & 0.01493372 & 0.033210184 & 0.019007118 & 0 & 0.011544242 & 0.021068612 & 0.016826559 & 0.037973478 & 0.075321445 & 0.02632651 \\
\hline & January & 58 & 54 & 0.016758229 & 0.01493372 & 0.033210184 & 0.019007118 & 0 & 0.011544242 & 0.021068612 & 0.016826559 & 0.037973478 & 0.075321445 & 0.02632651 \\
\hline \multirow{12}{*}{2013} & December & 43 & 55 & 0.016827088 & 0.014866932 & 0.033547645 & 0.018948439 & 0 & 0.011571128 & 0.021029546 & 0.016859427 & 0.03787813 & 0.07552179 & 0.026329933 \\
\hline & November & 34 & 56 & 0.016827088 & 0.014866932 & 0.033547645 & 0.018948439 & 0 & 0.011571128 & 0.021029546 & 0.016859427 & 0.03787813 & 0.07552179 & 0.026329933 \\
\hline & October & 25 & 57 & 0.016827088 & 0.014866932 & 0.033547645 & 0.018948439 & 0 & 0.011571128 & 0.021029546 & 0.016859427 & 0.03787813 & 0.07552179 & 0.026329933 \\
\hline & September & 12 & 58 & 0.016827088 & 0.014866932 & 0.033547645 & 0.018948439 & 0 & 0.011571128 & 0.021029546 & 0.016859427 & 0.03787813 & 0.07552179 & 0.026329933 \\
\hline & August & 6 & 59 & 0.016814453 & 0.01484884 & 0.033609414 & 0.018943859 & 0 & 0.011573638 & 0.021036864 & 0.016855872 & 0.03786978 & 0.07560387 & 0.026328199 \\
\hline & July & 14 & 60 & 0.016835562 & 0.014847654 & 0.033668603 & 0.01891872 & 0 & 0.011576148 & 0.021050562 & 0.016831846 & 0.03784747 & 0.075590568 & 0.026302586 \\
\hline & June & 19 & 61 & 0.016835562 & 0.014847654 & 0.033668603 & 0.01891872 & 0 & 0.011576148 & 0.021050562 & 0.016831846 & 0.03784747 & 0.075590568 & 0.026302586 \\
\hline & May & 48 & 62 & 0.016841305 & 0.014841183 & 0.03366147 & 0.018926727 & 0 & 0.011578648 & 0.02104386 & 0.016855245 & 0.03784849 & 0.075608584 & 0.02632144 \\
\hline & April & 51 & 63 & 0.016841305 & 0.014841183 & 0.03366147 & 0.018926727 & 0 & 0.011578648 & 0.02104386 & 0.016855245 & 0.03784849 & 0.075608584 & 0.02632144 \\
\hline & March & 45 & 64 & 0.016860416 & 0.014834272 & 0.033705579 & 0.018918364 & 0 & 0.011581863 & 0.021029498 & 0.016868078 & 0.03783197 & 0.075626861 & 0.0263261 \\
\hline & February & 40 & 65 & 0.01686496 & 0.014827191 & 0.033729594 & 0.018914824 & 0 & 0.011585938 & 0.021034788 & 0.016871461 & 0.03782549 & 0.075647992 & 0.026327972 \\
\hline & Sum & & & 0.868423084 & 0.821709848 & 1.958708773 & 1.228795753 & & 2.331576772 & 1.146078895 & 1.047170583 & 2.379373736 & 6.850911919 & 1.603967641 \\
\hline
\end{tabular}




\begin{tabular}{|c|c|c|c|c|c|c|c|c|c|c|c|c|c|c|}
\hline & & Initial State & Transition & State 45 & State 46 & State 47 & State 48 & State 49 & State 50 & State 51 & State 52 & State 53 & State 54 & State 55 \\
\hline \multirow{6}{*}{2018} & June & 57 & 1 & 0 & 0 & 0 & 0 & 0 & 0 & 0 & 0 & 0 & 0 & 0 \\
\hline & May & 50 & 2 & 0 & 0 & 0 & 0 & 0 & 0 & 0 & 0 & 0 & 0 & 0 \\
\hline & April & 31 & 3 & 0 & 0 & 0 & 0 & 0 & 0.148148 & 0 & 0 & 0 & 0 & 0 \\
\hline & March & 31 & 4 & 0 & 0 & 0 & 0 & 0 & 0.08642 & 0 & 0 & 0 & 0 & 0 \\
\hline & February & 27 & 5 & 0 & 0 & 0 & 0 & 0 & 0.08642 & 0 & 0 & 0 & 0 & 0 \\
\hline & January & 31 & 6 & 0.020833 & 0.020833 & 0.041667 & 0.020833 & 0 & 0.05487 & 0 & 0 & 0.034722 & 0 & 0 \\
\hline \multirow{12}{*}{2017} & December & 47 & 7 & 0.013696 & 0.012153 & 0.024306 & 0.016782 & 0 & 0.02412 & 0 & 0 & 0.016782 & 0 & 0.001543 \\
\hline & November & 40 & 8 & 0.012056 & 0.049045 & 0.017216 & 0.008391 & 0.002315 & 0.021405 & 0.005064 & 0 & 0.02228 & 0 & 0.00598 \\
\hline & October & 39 & 9 & 0.012056 & 0.049045 & 0.017216 & 0.008391 & 0.002315 & 0.021405 & 0.005064 & 0 & 0.02228 & 0 & 0.00598 \\
\hline & September & 18 & 10 & 0.012056 & 0.049045 & 0.017216 & 0.008391 & 0.002315 & 0.021405 & 0.005064 & 0 & 0.02228 & 0 & 0.00598 \\
\hline & August & 16 & 11 & 0.012056 & 0.049045 & 0.017216 & 0.008391 & 0.002315 & 0.021405 & 0.005064 & 0 & 0.02228 & 0 & 0.00598 \\
\hline & July & 22 & 12 & 0.012056 & 0.049045 & 0.017216 & 0.008391 & 0.002315 & 0.021405 & 0.005064 & 0 & 0.02228 & 0 & 0.00598 \\
\hline & June & 32 & 13 & 0.012056 & 0.049045 & 0.017216 & 0.008391 & 0.002315 & 0.021405 & 0.005064 & 0 & 0.02228 & 0 & 0.00598 \\
\hline & May & 49 & 14 & 0.012056 & 0.049045 & 0.017216 & 0.008391 & 0.002315 & 0.021405 & 0.005064 & 0 & 0.02228 & 0 & 0.00598 \\
\hline & April & 55 & 15 & 0.012056 & 0.049045 & 0.017216 & 0.008391 & 0.002315 & 0.021405 & 0.005064 & 0 & 0.02228 & 0 & 0.00598 \\
\hline & March & 47 & 16 & 0.015941 & 0.041805 & 0.02473 & 0.015811 & 0.006772 & 0.016335 & 0.006653 & 0 & 0.029729 & 0 & 0.007306 \\
\hline & February & 45 & 17 & 0.0198 & 0.032933 & 0.039913 & 0.028582 & 0.009245 & 0.012819 & 0.007562 & 0 & 0.033728 & 0 & 0.007647 \\
\hline & January & 47 & 18 & 0.044646 & 0.032707 & 0.059335 & 0.024391 & 0.008243 & 0.013019 & 0.007183 & 0 & 0.032773 & 0 & 0.008915 \\
\hline \multirow{12}{*}{2016} & December & 44 & 19 & 0.033327 & 0.037521 & 0.045577 & 0.024184 & 0.009246 & 0.011635 & 0.008269 & 0 & 0.037115 & 0 & 0.009639 \\
\hline & November & 36 & 20 & 0.026271 & 0.038193 & 0.040838 & 0.024318 & 0.00985 & 0.011448 & 0.010201 & 0 & 0.038246 & 0 & 0.010419 \\
\hline & October & 35 & 21 & 0.026271 & 0.038193 & 0.040838 & 0.024318 & 0.00985 & 0.011448 & 0.010201 & 0 & 0.038246 & 0 & 0.010419 \\
\hline & September & 14 & 22 & 0.024317 & 0.039861 & 0.037629 & 0.024859 & 0.011379 & 0.011132 & 0.010064 & 0 & 0.039351 & 0 & 0.010242 \\
\hline & August & 15 & 23 & 0.024317 & 0.039861 & 0.037629 & 0.024859 & 0.011379 & 0.011132 & 0.010064 & 0 & 0.039351 & 0 & 0.010242 \\
\hline & July & 17 & 24 & 0.024317 & 0.039861 & 0.037629 & 0.024859 & 0.011379 & 0.011132 & 0.010064 & 0 & 0.039351 & 0 & 0.010242 \\
\hline & June & 29 & 25 & 0.024317 & 0.039861 & 0.037629 & 0.024859 & 0.011379 & 0.011132 & 0.010064 & 0 & 0.039351 & 0 & 0.010242 \\
\hline & May & 46 & 26 & 0.024472 & 0.03698 & 0.036668 & 0.024326 & 0.011068 & 0.010886 & 0.00997 & 0 & 0.038152 & 0 & 0.011074 \\
\hline & April & 53 & 27 & 0.023023 & 0.036407 & 0.034562 & 0.024667 & 0.011902 & 0.01123 & 0.010594 & 0 & 0.037846 & 0 & 0.011409 \\
\hline & March & 42 & 28 & 0.022394 & 0.036382 & 0.034559 & 0.023972 & 0.011628 & 0.011069 & 0.010882 & 0 & 0.037339 & 0 & 0.011519 \\
\hline & February & 42 & 29 & 0.02176 & 0.036496 & 0.034665 & 0.02411 & 0.011519 & 0.011166 & 0.011197 & 0 & 0.037038 & 0 & 0.01152 \\
\hline & January & 43 & 30 & 0.021853 & 0.036873 & 0.0344 & 0.022922 & 0.011387 & 0.011454 & 0.011151 & 0 & 0.03671 & 0 & 0.011466 \\
\hline \multirow{12}{*}{2015} & December & 41 & 31 & 0.021853 & 0.036873 & 0.0344 & 0.022922 & 0.011387 & 0.011454 & 0.011151 & 0 & 0.03671 & 0 & 0.011466 \\
\hline & November & 36 & 32 & 0.022639 & 0.035892 & 0.035327 & 0.024042 & 0.011834 & 0.011636 & 0.01148 & 0 & 0.036712 & 0 & 0.011784 \\
\hline & October & 30 & 33 & 0.022639 & 0.035892 & 0.035327 & 0.024042 & 0.011834 & 0.011636 & 0.01148 & 0 & 0.036712 & 0 & 0.011784 \\
\hline & September & 4 & 34 & 0.022639 & 0.035892 & 0.035327 & 0.024042 & 0.011834 & 0.011636 & 0.01148 & 0 & 0.036712 & 0 & 0.011784 \\
\hline & August & 1 & 35 & 0.022639 & 0.035892 & 0.035327 & 0.024042 & 0.011834 & 0.011636 & 0.01148 & 0 & 0.036712 & 0 & 0.011784 \\
\hline & July & 10 & 36 & 0.022639 & 0.035892 & 0.035327 & 0.024042 & 0.011834 & 0.011636 & 0.01148 & 0 & 0.036712 & 0 & 0.011784 \\
\hline & June & 13 & 37 & 0.022268 & 0.035785 & 0.035102 & 0.024203 & 0.011688 & 0.011548 & 0.011273 & 0 & 0.036806 & 0 & 0.011577 \\
\hline & May & 43 & 38 & 0.021809 & 0.035579 & 0.03468 & 0.024288 & 0.011629 & 0.011591 & 0.011071 & 0 & 0.036637 & 0 & 0.011418 \\
\hline & April & 48 & 39 & 0.021904 & 0.0353 & 0.034699 & 0.02434 & 0.011566 & 0.011561 & 0.011026 & 0 & 0.036608 & 0 & 0.011459 \\
\hline & March & 44 & 40 & 0.022429 & 0.034952 & 0.03515 & 0.024462 & 0.01167 & 0.0116 & 0.011211 & 0 & 0.036491 & 0 & 0.011683 \\
\hline & February & 37 & 41 & 0.022579 & 0.034971 & 0.035278 & 0.024399 & 0.011683 & 0.011603 & 0.011279 & 0 & 0.036463 & 0 & 0.011743 \\
\hline & January & 43 & 42 & 0.022151 & 0.035149 & 0.034913 & 0.024091 & 0.011576 & 0.011548 & 0.011146 & 0 & 0.0364 & 0 & 0.01162 \\
\hline \multirow{12}{*}{2014} & December & 57 & 43 & 0.022151 & 0.035149 & 0.034913 & 0.024091 & 0.011576 & 0.011548 & 0.011146 & 0 & 0.0364 & 0 & 0.01162 \\
\hline & November & 53 & 44 & 0.022004 & 0.035001 & 0.034801 & 0.024224 & 0.011572 & 0.011549 & 0.01106 & 0 & 0.036392 & 0 & 0.011563 \\
\hline & October & 46 & 45 & 0.022133 & 0.035195 & 0.034772 & 0.024085 & 0.011563 & 0.011547 & 0.011075 & 0 & 0.036466 & 0 & 0.011592 \\
\hline & September & 9 & 46 & 0.022133 & 0.035195 & 0.034772 & 0.024085 & 0.011563 & 0.011547 & 0.011075 & 0 & 0.036466 & 0 & 0.011592 \\
\hline & August & 6 & 47 & 0.022193 & 0.035226 & 0.034735 & 0.024119 & 0.011591 & 0.011553 & 0.011075 & 0 & 0.036516 & 0 & 0.011615 \\
\hline & July & 13 & 48 & 0.022171 & 0.034993 & 0.034765 & 0.024243 & 0.011586 & 0.011565 & 0.011028 & 0 & 0.036452 & 0 & 0.011606 \\
\hline & June & 26 & 49 & 0.022171 & 0.034993 & 0.034765 & 0.024243 & 0.011586 & 0.011565 & 0.011028 & 0 & 0.036452 & 0 & 0.011606 \\
\hline & May & 61 & 50 & 0.022171 & 0.034993 & 0.034765 & 0.024243 & 0.011586 & 0.011565 & 0.011028 & 0 & 0.036452 & 0 & 0.011606 \\
\hline & April & 65 & 51 & 0.022171 & 0.034993 & 0.034765 & 0.024243 & 0.011586 & 0.011565 & 0.011028 & 0 & 0.036452 & 0 & 0.011606 \\
\hline & March & 59 & 52 & 0.022171 & 0.034993 & 0.034765 & 0.024243 & 0.011586 & 0.011565 & 0.011028 & 0 & 0.036452 & 0 & 0.011606 \\
\hline & February & 56 & 53 & 0.022171 & 0.034993 & 0.034765 & 0.024243 & 0.011586 & 0.011565 & 0.011028 & 0 & 0.036452 & 0 & 0.011606 \\
\hline & January & 58 & 54 & 0.022171 & 0.034993 & 0.034765 & 0.024243 & 0.011586 & 0.011565 & 0.011028 & 0 & 0.036452 & 0 & 0.011606 \\
\hline \multirow{13}{*}{2013} & December & 43 & 55 & 0.022102 & 0.035058 & 0.034714 & 0.024212 & 0.011576 & 0.011572 & 0.011032 & 0 & 0.036455 & 0 & 0.011581 \\
\hline & November & 34 & 56 & 0.022102 & 0.035058 & 0.034714 & 0.024212 & 0.011576 & 0.011572 & 0.011032 & 0 & 0.036455 & 0 & 0.011581 \\
\hline & October & 25 & 57 & 0.022102 & 0.035058 & 0.034714 & 0.024212 & 0.011576 & 0.011572 & 0.011032 & 0 & 0.036455 & 0 & 0.011581 \\
\hline & September & 12 & 58 & 0.022102 & 0.035058 & 0.034714 & 0.024212 & 0.011576 & 0.011572 & 0.011032 & 0 & 0.036455 & 0 & 0.011581 \\
\hline & August & 6 & 59 & 0.02209 & 0.03506 & 0.034722 & 0.024214 & 0.011574 & 0.011571 & 0.011037 & 0 & 0.036447 & 0 & 0.011578 \\
\hline & July & 14 & 60 & 0.022093 & 0.035091 & 0.034728 & 0.024204 & 0.011574 & 0.011572 & 0.011042 & 0 & 0.036457 & 0 & 0.011575 \\
\hline & June & 19 & 61 & 0.022093 & 0.035091 & 0.034728 & 0.024204 & 0.011574 & 0.011572 & 0.011042 & 0 & 0.036457 & 0 & 0.011575 \\
\hline & May & 48 & 62 & 0.022094 & 0.035079 & 0.034726 & 0.024208 & 0.011572 & 0.011572 & 0.011039 & 0 & 0.036455 & 0 & 0.011575 \\
\hline & April & 51 & 63 & 0.022094 & 0.035079 & 0.034726 & 0.024208 & 0.011572 & 0.011572 & 0.011039 & 0 & 0.036455 & 0 & 0.011575 \\
\hline & arch & 45 & 64 & 22083 & 0.035089 & 0.03471 & 0.024202 & 0.011569 & 0.011574 & 0.011035 & 0 & 0.036458 & 0 & 0.01157 \\
\hline & February & 40 & 65 & 0.022077 & 0.035095 & 0.034709 & 0.0242 & 0.011567 & 0.011574 & 0.011035 & 0 & 0.036458 & 0 & 0.011568 \\
\hline & & & & & & & & & & & & & & \\
\hline & Sum & & & 1.28504 & 2.223882 & 2.000418 & 1.311291 & 0.581386 & 1.153334 & 0.574207 & & 2.062158 & 0 & 0.608136 \\
\hline
\end{tabular}




\begin{tabular}{|c|c|c|c|c|c|c|c|c|c|c|c|c|c|}
\hline & & Initial State & Transition & State 56 & State 57 & State 58 & State 59 & State 60 & State 61 & State 62 & State 63 & State 64 & State 65 \\
\hline \multirow{6}{*}{2018} & June & 57 & 1 & 0 & 0 & 0 & 0 & 0 & 0 & 0 & 0 & 0 & 0 \\
\hline & May & 50 & 2 & 0 & 0 & 0 & 0 & 0 & 0 & 0 & 0 & 0 & 0 \\
\hline & April & 31 & 3 & 0 & 0.111111 & 0 & 0 & 0 & 0 & 0 & 0 & 0 & 0 \\
\hline & March & 31 & 4 & 0 & 0.148148 & 0.083333 & 0 & 0 & 0 & 0 & 0 & 0 & 0 \\
\hline & February & 27 & 5 & 0 & 0.148148 & 0.083333 & 0 & 0 & 0 & 0 & 0 & 0 & 0 \\
\hline & January & 31 & 6 & 0.027778 & 0.099023 & 0.037037 & 0.083333 & 0 & 0 & 0 & 0 & 0 & 0 \\
\hline \multirow{12}{*}{2017} & December & 47 & 7 & 0.009259 & 0.049533 & 0.020448 & 0.048611 & 0 & 0 & 0 & 0 & 0 & 0 \\
\hline & November & 40 & 8 & 0.016782 & 0.037417 & 0.012539 & 0.024306 & 0 & 0 & 0 & 0 & 0 & 0.03125 \\
\hline & October & 39 & 9 & 0.016782 & 0.037417 & 0.012539 & 0.024306 & 0 & 0 & 0 & 0 & 0 & 0.03125 \\
\hline & September & 18 & 10 & 0.016782 & 0.037417 & 0.012539 & 0.024306 & 0 & 0 & 0 & 0 & 0 & \\
\hline & August & 16 & 11 & 0.016782 & 0.037417 & 0.012539 & 0.024306 & 0 & 0 & 0 & 0 & 0 & 0.03125 \\
\hline & July & 22 & 12 & 0.016782 & 0.037417 & 0.012539 & 0.024306 & 0 & 0 & 0 & 0 & 0 & 0.03125 \\
\hline & June & 32 & 13 & 0.016782 & 0.037417 & 0.012539 & 0.024306 & 0 & 0 & 0 & 0 & 0 & 0.03125 \\
\hline & May & 49 & 14 & 0.016782 & 0.037417 & 0.012539 & 0.024306 & 0 & 0 & 0 & 0 & 0 & 0.03125 \\
\hline & April & 55 & 15 & 0.016782 & 0.037417 & 0.012539 & 0.024306 & 0 & 0 & 0 & 0 & 0 & 0.03125 \\
\hline & March & 47 & 16 & 0.01684 & 0.037251 & 0.01458 & 0.018334 & 0 & 0.011103 & 0 & 0 & 0 & 0.023054 \\
\hline & February & 45 & 17 & 0.023444 & 0.030313 & 0.018204 & 0.016207 & 0 & 0.012665 & 0 & 0 & 0 & 0.016766 \\
\hline & January & 47 & 18 & 0.01926 & 0.032981 & 0.018307 & 0.01458 & 0 & 0.018334 & 0 & 0 & 0 & 0.01684 \\
\hline \multirow{12}{*}{2016} & December & 44 & 19 & 0.01775 & 0.032679 & 0.015991 & 0.018878 & 0 & 0.024191 & 0 & 0 & 0 & 0.019334 \\
\hline & November & 36 & 20 & 0.016643 & 0.031708 & 0.014546 & 0.018443 & 0 & 0.021389 & 0 & 0 & 0 & 0.024306 \\
\hline & October & 35 & 21 & 0.016643 & 0.031708 & 0.014546 & 0.018443 & 0 & 0.021389 & 0 & 0 & 0 & 0.024306 \\
\hline & September & 14 & 22 & 0.01833 & 0.030164 & 0.015408 & 0.02181 & 0 & 0.023039 & 0 & 0 & 0 & 0.02297 \\
\hline & August & 15 & 23 & 0.01833 & 0.030164 & 0.015408 & 0.02181 & 0 & 0.023039 & 0 & 0 & 0 & 0.02297 \\
\hline & July & 17 & 24 & 0.01833 & 0.030164 & 0.015408 & 0.02181 & 0 & 0.023039 & 0 & 0 & 0 & 0.02297 \\
\hline & June & 29 & 25 & 0.01833 & 0.030164 & 0.015408 & 0.02181 & 0 & 0.023039 & 0 & 0 & 0 & 0.02297 \\
\hline & May & 46 & 26 & 0.019058 & 0.029657 & 0.01771 & 0.021614 & 0 & 0.02083 & 0 & 0 & 0 & 0.020416 \\
\hline & April & 53 & 27 & 0.017548 & 0.029609 & 0.018358 & 0.020012 & 0 & 0.021417 & 0 & 0 & 0 & 0.019785 \\
\hline & March & 42 & 28 & 0.018711 & 0.029325 & 0.018534 & 0.019874 & 0 & 0.019687 & 0 & 0 & 0 & 0.019736 \\
\hline & February & 42 & 29 & 0.018534 & 0.029739 & 0.018132 & 0.018711 & 0 & 0.019736 & 0 & 0 & 0 & 0.019874 \\
\hline & January & 43 & 30 & 0.019314 & 0.029978 & 0.017845 & 0.019526 & 0 & 0.01798 & 0 & 0 & 0 & 0.021218 \\
\hline \multirow{12}{*}{2015} & December & 41 & 31 & 0.019314 & 0.029978 & 0.017845 & 0.019526 & 0 & 0.01798 & 0 & 0 & 0 & 0.021218 \\
\hline & November & 36 & 32 & 0.019213 & 0.029919 & 0.018072 & 0.019618 & 0 & 0.018508 & 0 & 0 & 0 & 0.020163 \\
\hline & October & 30 & 33 & 0.019213 & 0.029919 & 0.018072 & 0.019618 & 0 & 0.018508 & 0 & 0 & 0 & 0.020163 \\
\hline & September & 4 & 34 & 0.019213 & 0.029919 & 0.018072 & 0.019618 & 0 & 0.018508 & 0 & 0 & 0 & 0.020163 \\
\hline & August & 1 & 35 & 0.019213 & 0.029919 & 0.018072 & 0.019618 & 0 & 0.018508 & 0 & 0 & 0 & 0.020163 \\
\hline & July & 10 & 36 & 0.019213 & 0.029919 & 0.018072 & 0.019618 & 0 & 0.018508 & 0 & 0 & 0 & 0.020163 \\
\hline & June & 13 & 37 & 0.018833 & 0.029901 & 0.018233 & 0.018959 & 0 & 0.019183 & 0 & 0 & 0 & 0.019754 \\
\hline & May & 43 & 38 & 0.018777 & 0.029941 & 0.018422 & 0.018631 & 0 & 0.019242 & 0 & 0 & 0 & 0.019278 \\
\hline & April & 48 & 39 & 0.018627 & 0.029943 & 0.018628 & 0.018547 & 0 & 0.019389 & 0 & 0 & 0 & 0.018993 \\
\hline & March & 44 & 40 & 0.018774 & 0.029875 & 0.01882 & 0.018812 & 0 & 0.019092 & 0 & 0 & 0 & 0.018807 \\
\hline & February & 37 & 41 & 0.018876 & 0.029834 & 0.018846 & 0.018926 & 0 & 0.018937 & 0 & 0 & 0 & 0.018873 \\
\hline & January & 43 & 42 & 0.01922 & 0.029712 & 0.018903 & 0.01902 & 0 & 0.018422 & 0 & 0 & 0 & 0.019069 \\
\hline \multirow{12}{*}{2014} & December & 57 & 43 & 0.01922 & 0.029712 & 0.018903 & 0.01902 & 0 & 0.018422 & 0 & 0 & 0 & 0.019069 \\
\hline & November & 53 & 44 & 0.019132 & 0.029722 & 0.018984 & 0.018859 & 0 & 0.018564 & 0 & 0 & 0 & 0.018881 \\
\hline & October & 46 & 45 & 0.019064 & 0.029732 & 0.018899 & 0.019064 & 0 & 0.018699 & 0 & 0 & 0 & 0.019044 \\
\hline & September & 9 & 46 & 0.019064 & 0.029732 & 0.018899 & 0.019064 & 0 & 0.018699 & 0 & 0 & 0 & 0.019044 \\
\hline & August & 6 & 47 & 0.018984 & 0.02974 & 0.018894 & 0.019142 & 0 & 0.018884 & 0 & 0 & 0 & 0.019032 \\
\hline & July & 13 & 48 & 0.019 & 0.029744 & 0.019007 & 0.019043 & 0 & 0.018839 & 0 & 0 & 0 & 0.018815 \\
\hline & June & 26 & 49 & 0.019 & 0.029744 & 0.019007 & 0.019043 & 0 & 0.018839 & 0 & 0 & 0 & 0.018815 \\
\hline & May & 61 & 50 & 0.019 & 0.029744 & 0.019007 & 0.019043 & 0 & 0.018839 & 0 & 0 & 0 & 0.018815 \\
\hline & April & 65 & 51 & 0.019 & 0.029744 & 0.019007 & 0.019043 & 0 & 0.018839 & 0 & 0 & 0 & 0.018815 \\
\hline & March & 59 & 52 & 0.019 & 0.029744 & 0.019007 & 0.019043 & 0 & 0.018839 & 0 & 0 & 0 & 0.018815 \\
\hline & February & 56 & 53 & 0.019 & 0.029744 & 0.019007 & 0.019043 & 0 & 0.018839 & 0 & 0 & 0 & 0.018815 \\
\hline & January & 58 & 54 & 0.019 & 0.029744 & 0.019007 & 0.019043 & 0 & 0.018839 & 0 & 0 & 0 & 0.018815 \\
\hline \multirow{11}{*}{2013} & December & 43 & 55 & 0.018941 & 0.029785 & 0.018948 & 0.018959 & 0 & 0.018884 & 0 & 0 & 0 & 0.018872 \\
\hline & November & 34 & 56 & 0.018941 & 0.029785 & 0.018948 & 0.018959 & 0 & 0.018884 & 0 & 0 & 0 & 0.018872 \\
\hline & October & 25 & 57 & 0.018941 & 0.029785 & 0.018948 & 0.018959 & 0 & 0.018884 & 0 & 0 & 0 & 0.018872 \\
\hline & September & 12 & 58 & 0.018941 & 0.029785 & 0.018948 & 0.018959 & 0 & 0.018884 & 0 & 0 & 0 & 0.018872 \\
\hline & August & 6 & 59 & 0.018945 & 0.029787 & 0.018944 & 0.018933 & 0 & 0.018864 & 0 & 0 & 0 & 0.018879 \\
\hline & July & 14 & 60 & 0.018941 & 0.029792 & 0.018919 & 0.018935 & 0 & 0.018876 & 0 & 0 & 0 & 0.01891 \\
\hline & June & 19 & 61 & 0.018941 & 0.029792 & 0.018919 & 0.018935 & 0 & 0.018876 & 0 & 0 & 0 & 0.01891 \\
\hline & May & 48 & 62 & 0.018932 & 0.029794 & 0.018927 & 0.018928 & 0 & 0.018885 & 0 & 0 & 0 & 0.018895 \\
\hline & April & 51 & 63 & 0.018932 & 0.029794 & 0.018927 & 0.018928 & 0 & 0.018885 & 0 & 0 & 0 & 0.018895 \\
\hline & March & 45 & 64 & 0.018916 & 0.029802 & 0.018918 & 0.018919 & 0 & 0.018904 & 0 & 0 & 0 & 0.018901 \\
\hline & February & 40 & 65 & 0.018913 & 0.029804 & 0.018915 & 0.018909 & 0 & 0.018906 & 0 & 0 & 0 & 0.018906 \\
\hline & Sum & & & 1.113594 & 2.364233 & 1.228796 & 1.285535 & & 0.958533 & & 0 & 0 & 1.207629 \\
\hline
\end{tabular}


$492,787,024.62 \times$

$\left(1+\frac{3.46036396884151+4.08196103290961}{2} \%\right)$

$=511370824.098922$

Then the forecasting value of original data sequence is obtained by:

$$
\hat{x}(k)=\hat{x}^{(0)}(k) \times\left(1+\frac{\otimes_{j-}+\otimes_{j+}}{2} \%\right)
$$

Then the mathematical model for the MCGM of monthly electricity consumption of NCR is:

$\hat{x}(k)=\hat{x}^{(0)}(k) \times\left(1+\frac{3.46036396884151+4.08196103290961}{2} \%\right)$

The forecast values from January 2013 to June 2018 are also calculated by Markov Chain Grey Model (see Table 6).

We will now solve the following for the forecasting accuracy: Mean Square Error (MSE), Root Mean Square Error (RMSE), Normalized Mean Square Error (NMSE), Mean Absolute Percentage Error (MAPE), and Mean Absolute Error (MAE).

For the Mean Square Error:

$$
M S E=\frac{1}{n} \sum_{i=1}^{n}\left(A_{i}-F_{i}\right)^{2}
$$

Where $n=$ sample size, $y=$ observed values, and $\tilde{y}=$ forecasts.

$G M(1,1)$

$\operatorname{MCGM}(1,1)$

Then the MSE for GM $(1,1)$ and MCGM of monthly electricity consumption from January 2013 to June 2018 are:

$$
\begin{aligned}
& G M(1,1): \quad M S E=\frac{1}{66} \sum_{i=1}^{66}\left[x^{(0)}(i)-\hat{x}^{(0)}(i)\right]^{2}=1622803285137450 \\
& M C G M(1,1): \quad M S E=\frac{1}{66} \sum_{i=1}^{66}\left[x^{(0)}(i)-\hat{x}(i)\right]^{2}=35954337846890.2
\end{aligned}
$$

For the Root Mean Square Error:

$$
R M S E=(M S E)^{1 / 2}
$$

Then the RMSE for GM $(1,1)$ and MCGM of monthly electricity consumption from January 2013 to June 2018 are:

$G M(1,1): \quad R M S E=\left[\frac{1}{66} \sum_{i=1}^{66}\left(x^{(0)}(i)-\hat{x}^{(0)}(i)\right)^{2}\right]^{1 / 2}=40284032.632514$ $\operatorname{MCGM}(1,1): R M S E=\left[\frac{1}{66} \sum_{i=1}^{66}\left(x^{(0)}(i)-\hat{x}(i)\right)^{2}\right]^{1 / 2}=5996193.61319247$

For the Normalized Mean Square Error:

$$
N M S E=\frac{\sum_{i=1}^{n}\left(A_{t}-F_{t}\right)^{2}}{n(\bar{A} \times \bar{F})}
$$

Where $n=$ sample size, $y=$ observed values, and $\tilde{y}=$ forecasts $\bar{y}=$ mean of the observed values, and $\tilde{y}=$ mean of the forecasted values.

Then the NMSE for GM $(1,1)$ and MCGM of monthly electricity consumption from January 2013 to June 2018 are:

$$
\begin{aligned}
& G M(1,1): N M S E=\frac{\sum_{i=1}^{66}\left[x^{(0)}(i)-\hat{x}^{(0)}(i)\right]^{2}}{66\left(\overline{x^{(0)}} \times \overline{\hat{x}^{(0)}}\right)} \\
& =\frac{\sum_{i=1}^{66}\left[x^{(0)}(i)-\hat{x}^{(0)}(i)\right]^{2}}{66(420909198.223887 \times 419923427.796618)} \\
& =0.009181367 \\
& M C G M(1,1): N M S E \\
& =\frac{\sum_{i=1}^{66}\left[x^{(0)}(i)-\hat{x}^{(0)}(i)\right]^{2}}{66\left(\overline{x^{(0)}} \times \overline{\hat{X}}\right)} \\
& =\frac{\sum_{i=1}^{66}\left[x^{(0)}(i)-\hat{x}^{(0)}(i)\right]^{2}}{66(420909198.223887 \times 416803737.180564)} \\
& =0.00020494214343992
\end{aligned}
$$

For the Mean Absolute Percentage Error:

$$
M A P E=\frac{1}{n} \sum_{i=1}^{n}\left|\frac{A_{i}-F_{i}}{A_{i}}\right| \times 100
$$

Then the MAPE for GM $(1,1)$ and MCGM of monthly electricity consumption from January 2013 to June 2018 are: 
$G M(1,1): \quad M A P E=\frac{\pi}{66} \sum^{66}\left|\frac{x^{(0)}(i) \hat{x}^{(0)}(i)}{x^{()}(i)}\right| \times 100=8.35200235177034$ $\operatorname{MCGM}(1,1):$ MAPE $=\frac{-66}{66} \sum^{66}\left|\frac{x^{()}(i) \hat{x}(i)}{\hat{x}(i)}\right| \times 100=1.07394173903827$

For the Mean Absolute Error:

$$
M A E=\frac{1}{n} \sum_{i=1}^{n}\left|F_{i}-A_{i}\right|
$$

Table 1. Actual data of the National Capital Region
Then the MAE for GM $(1,1)$ and MCGM of monthly electricity consumption from January 2013 to June 2018 are:

$$
\begin{aligned}
& \operatorname{GM}(1,1): \quad M A E=\frac{1}{66} \sum_{i=1}^{66}\left|x^{(0)}(i)-\hat{x}^{(0)}(i)\right|=33974305.8325304 \\
& M C G M(1,1): M A E=\frac{1}{66} \sum_{i=1}^{66}\left|x^{(0)}(i)-\hat{x}(i)\right|=4227582.03151131
\end{aligned}
$$

\begin{tabular}{|l|l|l|l|l|l|l|l|l|l|}
\hline $\mathrm{k}$ & & $\mathrm{k}$ & & $\mathrm{k}$ & & $\mathrm{k}$ & & $\mathrm{k}$ & \\
\hline 1 & $368,026,507.90$ & 15 & $5,615,697,933.68$ & 29 & $11,338,776,539.61$ & 43 & $17,072,284,836.84$ & 57 & $23,365,745,197.05$ \\
\hline 2 & $735,710,143.21$ & 16 & $6,083,660,114.95$ & 30 & $11,697,255,018.35$ & 44 & $17,459,207,003.56$ & 58 & $23,844,775,296.23$ \\
\hline 3 & $1,115,972,908.54$ & 17 & $6,539,285,276.18$ & 31 & $12,051,589,661.85$ & 45 & $17,847,054,186.68$ & 59 & $24,327,222,679.00$ \\
\hline 4 & $1,514,059,499.61$ & 18 & $6,903,894,594.39$ & 32 & $12,390,692,740.79$ & 46 & $18,287,264,889.55$ & 60 & $24,835,161,966.09$ \\
\hline 5 & $1,906,847,784.63$ & 19 & $7,244,372,693.70$ & 33 & $12,738,128,985.57$ & 47 & $18,731,392,185.73$ & 61 & $25,298,127,347.99$ \\
\hline 6 & $2,237,134,694.68$ & 20 & $7,574,124,886.62$ & 34 & $13,140,662,007.21$ & 48 & $19,200,806,941.30$ & 62 & $25,752,309,728.57$ \\
\hline 7 & $2,560,291,609.25$ & 21 & $7,910,543,335.76$ & 35 & $13,560,378,631.07$ & 49 & $19,682,102,024.29$ & 63 & $26,218,020,430.26$ \\
\hline 8 & $2,870,148,867.99$ & 22 & $8,331,635,278.42$ & 36 & $13,995,126,073.88$ & 50 & $20,161,205,828.81$ & 64 & $26,685,284,542.22$ \\
\hline 9 & $3,192,010,650.41$ & 23 & $8,773,145,184.39$ & 37 & $14,438,654,425.83$ & 51 & $20,647,631,355.39$ & 65 & $27,218,196,969.80$ \\
\hline 10 & $3,539,219,037.52$ & 24 & $9,231,117,812.62$ & 38 & $14,879,403,352.55$ & 52 & $21,162,813,546.21$ & 66 & $27,780,007,082.78$ \\
\hline 11 & $3,908,261,772.63$ & 25 & $9,648,016,839.14$ & 39 & $15,323,883,084.24$ & 53 & $21,659,922,272.42$ & & \\
\hline 12 & $4,301,309,518.23$ & 26 & $10,051,265,533.32$ & 40 & $15,803,768,142.46$ & 54 & $22,108,318,131.88$ & & \\
\hline 13 & $4,737,346,468.98$ & 27 & $10,474,898,303.09$ & 41 & $16,264,630,162.48$ & 55 & $22,532,587,345.05$ & & \\
\hline 14 & $5,170,211,112.79$ & 28 & $10,912,994,948.09$ & 42 & $16,681,481,847.90$ & 56 & $22,946,028,969.15$ & & \\
\hline
\end{tabular}

Table 2. 1-AGO of the actual data of the National Capital Region

\begin{tabular}{|l|l|l|l|l|l|l|l|l|l|}
\hline $\mathbf{k}$ & $\begin{array}{l}\text { Actual Data } \\
\left(\boldsymbol{x}^{(\mathbf{0})}(\boldsymbol{k})\right)\end{array}$ & $\mathbf{k}$ & $\begin{array}{l}\text { Actual Data } \\
\left(\boldsymbol{x}^{(\mathbf{0}}(\boldsymbol{k})\right)\end{array}$ & $\mathbf{K}$ & $\begin{array}{l}\text { Actual Data } \\
\left(\boldsymbol{x}^{(\mathbf{0})}(\boldsymbol{k})\right)\end{array}$ & $\mathbf{k}$ & $\begin{array}{l}\text { Actual Data } \\
\left(\boldsymbol{x}^{(\mathbf{0})}(\boldsymbol{k})\right)\end{array}$ & $\begin{array}{l}\text { Actual Data } \\
\left(\boldsymbol{x}^{(\mathbf{0})}(\boldsymbol{k})\right)\end{array}$ \\
\hline $\mathbf{1}$ & $368,026,507.90$ & $\mathbf{1 5}$ & $445,486,820.89$ & $\mathbf{2 9}$ & $425,781,591.52$ & $\mathbf{4 3}$ & $390,802,988.94$ & $\mathbf{5 7}$ & $419,716,227.90$ \\
\hline $\mathbf{2}$ & $367,683,635.31$ & $\mathbf{1 6}$ & $467,962,181.27$ & $\mathbf{3 0}$ & $358,478,478.74$ & $\mathbf{4 4}$ & $386,922,166.72$ & $\mathbf{5 8}$ & $479,030,099.18$ \\
\hline $\mathbf{3}$ & $380,262,765.33$ & $\mathbf{1 7}$ & $455,625,161.22$ & $\mathbf{3 1}$ & $354,334,643.50$ & $\mathbf{4 5}$ & $387,847,183.13$ & $\mathbf{5 9}$ & $482,447,382.77$ \\
\hline $\mathbf{4}$ & $398,086,591.07$ & $\mathbf{1 8}$ & $364,609,318.21$ & $\mathbf{3 2}$ & $339,103,078.94$ & $\mathbf{4 6}$ & $440,210,702.87$ & $\mathbf{6 0}$ & $507,939,287.09$ \\
\hline $\mathbf{5}$ & $392,788,285.02$ & $\mathbf{1 9}$ & $340,478,099.31$ & $\mathbf{3 3}$ & $347,436,244.78$ & $\mathbf{4 7}$ & $444,127,296.18$ & $\mathbf{6 1}$ & $462,965,381.90$ \\
\hline $\mathbf{6}$ & $330,286,910.05$ & $\mathbf{2 0}$ & $329,752,192.91$ & $\mathbf{3 4}$ & $402,533,021.64$ & $\mathbf{4 8}$ & $469,414,755.57$ & $\mathbf{6 2}$ & $454,182,380.58$ \\
\hline $\mathbf{7}$ & $323,156,914.57$ & $\mathbf{2 1}$ & $336,418,449.14$ & $\mathbf{3 5}$ & $419,716,623.86$ & $\mathbf{4 9}$ & $481,295,082.99$ & $\mathbf{6 3}$ & $465,710,701.69$ \\
\hline $\mathbf{8}$ & $309,857,258.74$ & $\mathbf{2 2}$ & $421,091,942.67$ & $\mathbf{3 6}$ & $434,747,442.81$ & $\mathbf{5 0}$ & $479,103,804.52$ & $\mathbf{6 4}$ & $467,264,111.96$ \\
\hline $\mathbf{9}$ & $321,861,782.42$ & $\mathbf{2 3}$ & $441,509,905.96$ & $\mathbf{3 7}$ & $443,528,351.96$ & $\mathbf{5 1}$ & $486,425,526.58$ & $\mathbf{6 5}$ & $532,912,427.58$ \\
\hline $\mathbf{1 0}$ & $347,208,387.12$ & $\mathbf{2 4}$ & $457,972,628.23$ & $\mathbf{3 8}$ & $440,748,926.72$ & $\mathbf{5 2}$ & $515,182,190.82$ & $\mathbf{6 6}$ & $561,810,112.98$ \\
\hline $\mathbf{1 1}$ & $369,042,735.11$ & $\mathbf{2 5}$ & $416,899,026.52$ & $\mathbf{3 9}$ & $444,479,731.69$ & $\mathbf{5 3}$ & $497,108,726.21$ & & \\
\hline $\mathbf{1 2}$ & $393,047,745.60$ & $\mathbf{2 6}$ & $403,248,694.18$ & $\mathbf{4 0}$ & $479,885,058.23$ & $\mathbf{5 4}$ & $448,395,859.46$ & & \\
\hline $\mathbf{1 3}$ & $436,036,950.75$ & $\mathbf{2 7}$ & $423,632,769.77$ & $\mathbf{4 1}$ & $460,862,020.02$ & $\mathbf{5 5}$ & $424,269,213.17$ & & \\
\hline $\mathbf{1 4}$ & $432,864,643.81$ & $\mathbf{2 8}$ & $438,096,645.00$ & $\mathbf{4 2}$ & $416,851,685.42$ & $\mathbf{5 6}$ & $413,441,624.10$ & & \\
\hline
\end{tabular}


Table 3. $\hat{x}^{(1)}(k+1)$ and $\hat{x}^{(0)}(k+1)$ values of Table 1

\begin{tabular}{|c|c|c|c|c|c|}
\hline & $\hat{x}^{(1)}(k+1)$ & $\hat{x}^{(0)}(k+1)$ & & $\hat{x}^{(1)}(k+1)$ & $\hat{x}^{(0)}(k+1)$ \\
\hline 1 & $368,026,507.90$ & $368,026,507.90$ & 34 & $13,231,312,326.98$ & $419,949,871.01$ \\
\hline 2 & $729,822,489.82$ & $359,617,273.24$ & 35 & $13,652,404,213.64$ & $421,990,190.53$ \\
\hline 3 & $1,093,286,243.13$ & $361,364,467.84$ & 36 & $14,075,437,208.10$ & $424,040,422.92$ \\
\hline 4 & $1,458,425,455.75$ & $363,120,151.14$ & 37 & $14,500,420,258.27$ & $426,100,616.32$ \\
\hline 5 & $1,825,247,851.06$ & $364,884,364.41$ & 38 & $14,927,362,353.35$ & $428,170,819.14$ \\
\hline 6 & $2,193,761,188.03$ & $366,657,149.08$ & 39 & $15,356,272,523.94$ & $430,251,080.00$ \\
\hline 7 & $2,563,973,261.38$ & $368,438,546.79$ & 40 & $15,787,159,842.28$ & $432,341,447.78$ \\
\hline 8 & $2,935,891,901.80$ & $370,228,599.39$ & 41 & $16,220,033,422.43$ & $434,441,971.57$ \\
\hline 9 & $3,309,524,976.03$ & $372,027,348.94$ & 42 & $16,654,902,420.46$ & $436,552,700.73$ \\
\hline 10 & $3,684,880,387.12$ & $373,834,837.68$ & 43 & $17,091,776,034.66$ & $438,673,684.82$ \\
\hline 11 & $4,061,966,074.52$ & $375,651,108.07$ & 44 & $17,530,663,505.71$ & $440,804,973.69$ \\
\hline 12 & $4,440,790,014.28$ & $377,476,202.79$ & 45 & $17,971,574,116.88$ & $442,946,617.38$ \\
\hline 13 & $4,821,360,219.23$ & $379,310,164.69$ & 46 & $18,414,517,194.24$ & $445,098,666.22$ \\
\hline 14 & $5,203,684,739.13$ & $381,153,036.87$ & 47 & $18,859,502,106.86$ & $447,261,170.75$ \\
\hline 15 & $5,587,771,660.84$ & $383,004,862.62$ & 48 & $19,306,538,267.00$ & $449,434,181.77$ \\
\hline 16 & $5,973,629,108.53$ & $384,865,685.43$ & 49 & $19,755,635,130.28$ & $451,617,750.33$ \\
\hline 17 & $6,361,265,243.77$ & $386,735,549.01$ & 50 & $20,206,802,195.93$ & $453,811,927.73$ \\
\hline 18 & $6,750,688,265.79$ & $388,614,497.30$ & 51 & $20,660,049,006.97$ & $456,016,765.49$ \\
\hline 19 & $7,141,906,411.60$ & $390,502,574.43$ & 52 & $21,115,385,150.40$ & $458,232,315.43$ \\
\hline 20 & $7,534,927,956.18$ & $392,399,824.75$ & 53 & $21,572,820,257.42$ & $460,458,629.58$ \\
\hline 21 & $7,929,761,212.68$ & $394,306,292.82$ & 54 & $22,032,364,003.63$ & $462,695,760.24$ \\
\hline 22 & $8,326,414,532.53$ & $396,222,023.45$ & 55 & $22,494,026,109.22$ & $464,943,759.96$ \\
\hline 23 & $8,724,896,305.68$ & $398,147,061.61$ & 56 & $22,957,816,339.20$ & $467,202,681.55$ \\
\hline 24 & $9,125,214,960.77$ & $400,081,452.55$ & 57 & $23,423,744,503.57$ & $469,472,578.07$ \\
\hline 25 & $9,527,378,965.26$ & $402,025,241.69$ & 58 & $23,891,820,457.60$ & $471,753,502.85$ \\
\hline 26 & $9,931,396,825.66$ & $403,978,474.69$ & 59 & $24,362,054,101.93$ & $474,045,509.46$ \\
\hline 27 & $10,337,277,087.70$ & $405,941,197.45$ & 60 & $24,834,455,382.88$ & $476,348,651.75$ \\
\hline 28 & $10,745,028,336.50$ & $407,913,456.06$ & 61 & $25,309,034,292.61$ & $478,662,983.82$ \\
\hline 29 & $11,154,659,196.73$ & $409,895,296.86$ & 62 & $25,785,800,869.33$ & $480,988,560.04$ \\
\hline 30 & $11,566,178,332.86$ & $411,886,766.40$ & 63 & $26,264,765,197.54$ & $483,325,435.03$ \\
\hline 31 & $11,979,594,449.26$ & $413,887,911.47$ & 64 & $26,745,937,408.21$ & $485,673,663.68$ \\
\hline 32 & $12,394,916,290.45$ & $415,898,779.06$ & 65 & $27,229,327,679.03$ & $488,033,301.17$ \\
\hline 33 & $12,812,152,641.25$ & $417,919,416.42$ & 66 & $27,714,946,234.58$ & $490,404,402.91$ \\
\hline
\end{tabular}


Table 4. Relative Errors of GM $(1,1)$ of the monthly electricity consumption of NCR

\begin{tabular}{|c|c|c|c|c|c|c|c|c|c|c|c|}
\hline $\mathbf{k}$ & $\begin{array}{c}\text { Relative } \\
\text { Error(k) }\end{array}$ & $\mathbf{k}$ & $\begin{array}{c}\text { Relative } \\
\text { Error(k) }\end{array}$ & $\mathbf{k}$ & $\begin{array}{c}\text { Relative } \\
\text { Error(k) }\end{array}$ & $\mathbf{k}$ & $\begin{array}{c}\text { Relative } \\
\text { Error(k) }\end{array}$ & $\mathbf{k}$ & $\begin{array}{c}\text { Relative } \\
\text { Error(k) }\end{array}$ & $\mathbf{k}$ & $\begin{array}{c}\text { Relative } \\
\text { Error(k) }\end{array}$ \\
\hline $\mathbf{1}$ & - & 12 & 3.96 & 23 & 9.82 & 34 & -4.33 & 45 & -14.21 & 56 & -13 \\
\hline $\mathbf{2}$ & 2.19 & 13 & 13.01 & 24 & 12.64 & 35 & -0.54 & 46 & -1.11 & 57 & -11.85 \\
\hline $\mathbf{3}$ & 4.97 & 14 & 11.95 & 25 & 3.57 & 36 & 2.46 & 47 & -0.71 & 58 & 1.52 \\
\hline $\mathbf{4}$ & 8.78 & 15 & 14.03 & 26 & -0.18 & 37 & 3.93 & 48 & 4.26 & 59 & 1.74 \\
\hline $\mathbf{5}$ & 7.1 & 16 & 17.76 & 27 & 4.18 & 38 & 2.85 & 49 & 6.17 & 60 & 6.22 \\
\hline $\mathbf{6}$ & -11.01 & 17 & 15.12 & 28 & 6.89 & 39 & 3.2 & 50 & 5.28 & 61 & -3.39 \\
\hline $\mathbf{7}$ & -14.01 & 18 & -6.58 & 29 & 3.73 & 40 & 9.91 & 51 & 6.25 & 62 & -5.9 \\
\hline $\mathbf{8}$ & -19.48 & 19 & -14.69 & 30 & -14.9 & 41 & 5.73 & 52 & 11.05 & 63 & -3.78 \\
\hline $\mathbf{9}$ & -15.59 & 20 & -19 & 31 & -16.81 & 42 & -4.73 & 53 & 7.37 & 64 & -3.94 \\
\hline $\mathbf{1 0}$ & -7.67 & 21 & -17.21 & 32 & -22.65 & 43 & -12.25 & 54 & -3.19 & 65 & 8.42 \\
\hline $\mathbf{1 1}$ & -1.79 & 22 & 5.91 & 33 & -20.29 & 44 & -13.93 & 55 & -9.59 & 66 & 12.71 \\
\hline
\end{tabular}

Table 5. Intervals for each state

\begin{tabular}{|c|c|c|c|c|c|c|c|c|}
\hline State & Lower bound & Upper bound & State & Lower bound & Upper bound & State & Lower bound & Upper bound \\
\hline 1 & -22.64671272 & -22.02511566 & 23 & -8.971577313 & -8.349980248 & 45 & 4.703558097 & 5.325155161 \\
\hline 2 & -22.02511566 & -21.40351859 & 24 & -8.349980248 & -7.728383184 & 46 & 5.325155161 & 5.946752225 \\
\hline 3 & -21.40351859 & -20.78192153 & 25 & -7.728383184 & -7.10678612 & 47 & 5.946752225 & 6.568349289 \\
\hline 4 & -20.78192153 & -20.16032447 & 26 & -7.10678612 & -6.485189056 & 48 & 6.568349289 & 7.189946353 \\
\hline 5 & -20.16032447 & -19.5387274 & 27 & -6.485189056 & -5.863591992 & 49 & 7.189946353 & 7.811543417 \\
\hline 6 & -19.5387274 & -18.91713034 & 28 & -5.863591992 & -5.241994928 & 50 & 7.811543417 & 8.433140481 \\
\hline 7 & -18.91713034 & -18.29553327 & 29 & -5.241994928 & -4.620397864 & 51 & 8.433140481 & 9.054737545 \\
\hline 8 & -18.29553327 & -17.67393621 & 30 & -4.620397864 & -3.9988008 & 52 & 9.054737545 & 9.67633461 \\
\hline 9 & -17.67393621 & -17.05233915 & 31 & -3.9988008 & -3.377203736 & 53 & 9.67633461 & 10.29793167 \\
\hline 10 & -17.05233915 & -16.43074208 & 32 & -3.377203736 & -2.755606672 & 54 & 10.29793167 & 10.91952874 \\
\hline 11 & -16.43074208 & -15.80914502 & 33 & -2.755606672 & -2.134009608 & 55 & 10.91952874 & 11.5411258 \\
\hline 12 & -15.80914502 & -15.18754795 & 34 & -2.134009608 & -1.512412544 & 56 & 11.5411258 & 12.16272287 \\
\hline 13 & -15.18754795 & -14.56595089 & 35 & -1.512412544 & -0.89081548 & 57 & 12.16272287 & 12.78431993 \\
\hline 14 & -14.56595089 & -13.94435383 & 36 & -0.89081548 & -0.269218416 & 58 & 12.78431993 & 13.40591699 \\
\hline 15 & -13.94435383 & -13.32275676 & 37 & -0.269218416 & 0.352378649 & 59 & 13.40591699 & 14.02751406 \\
\hline 16 & -13.32275676 & -12.7011597 & 38 & 0.352378649 & 0.973975713 & 60 & 14.02751406 & 14.64911112 \\
\hline 17 & -12.7011597 & -12.07956263 & 39 & 0.973975713 & 1.595572777 & 61 & 14.64911112 & 15.27070819 \\
\hline 18 & -12.07956263 & -11.45796557 & 40 & 1.595572777 & 2.217169841 & 62 & 15.27070819 & 15.89230525 \\
\hline 19 & -11.45796557 & -10.8363685 & 41 & 2.217169841 & 2.838766905 & 63 & 15.89230525 & 16.51390231 \\
\hline 20 & -10.8363685 & -10.21477144 & 42 & 2.838766905 & 3.460363969 & 64 & 16.51390231 & 17.13549938 \\
\hline 21 & -10.21477144 & -9.593174377 & 43 & 3.460363969 & 4.081961033 & 65 & 17.13549938 & 17.75709644 \\
\hline 22 & -9.593174377 & -8.971577313 & 44 & 4.081961033 & 4.703558097 & 66 & 17.75709644 & 18.37869351 \\
\hline
\end{tabular}


Table 6. Markov-Chain Grey Model Prediction for the monthly electricity consumption of NCR

\begin{tabular}{|c|c|c|c|c|c|}
\hline Year & Month & & Year & Month & \\
\hline \multirow{6}{*}{2018} & July & $511,370,824.10$ & \multirow{12}{*}{2020} & January & $557,987,162.15$ \\
\hline & August & $513,855,310.82$ & & February & $560,698,133.57$ \\
\hline & September & $516,351,868.37$ & & March & $563,422,276.21$ \\
\hline & October & $518,860,555.41$ & & April & $566,159,654.05$ \\
\hline & November & $521,381,430.86$ & & May & $568,910,331.39$ \\
\hline & December & $523,914,553.94$ & & June & $571,674,372.85$ \\
\hline \multirow{12}{*}{2019} & January & $526,459,984.16$ & & July & $574,451,843.37$ \\
\hline & February & $529,017,781.32$ & & August & $577,242,808.17$ \\
\hline & March & $531,588,005.48$ & & September & $580,047,332.84$ \\
\hline & April & $534,170,717.04$ & & October & $582,865,483.24$ \\
\hline & May & $536,765,976.66$ & & November & $585,697,325.58$ \\
\hline & June & $539,373,845.31$ & & December & $588,542,926.38$ \\
\hline & July & $541,994,384.24$ & \multirow{6}{*}{2021} & January & $591,402,352.48$ \\
\hline & August & $544,627,655.02$ & & February & $594,275,671.06$ \\
\hline & September & $547,273,719.50$ & & March & $597,162,949.60$ \\
\hline & October & $549,932,639.84$ & & April & $600,064,255.95$ \\
\hline & November & $552,604,478.50$ & & May & $602,979,658.24$ \\
\hline & December & $555,289,298.25$ & & June & $605,909,224.97$ \\
\hline
\end{tabular}

\subsubsection{Mathematical Models}

- Mathematical Model for CAR

$\operatorname{GM}(1,1): \hat{x}^{(0)}(k+1)=\left(1-e^{-0.0035}\right)\left(x^{(0)}(1)+\frac{32328124.41}{0.0035}\right) e^{0.0035 k}$,

$$
(k=1,2, \ldots, n)
$$

MCGM: $\quad \hat{y}=\hat{x}^{(0)}(k+1)\left(1-\frac{2.0890+1.8366}{2} \%\right)$

- Mathematical Model for Region I

$\operatorname{GM}(1,1): \hat{x}^{(0)}(k+1)=\left(1-e^{-0.0045}\right)\left(x^{(0)}(1)+\frac{133789408.31}{0.0045}\right) e^{0.0045 k}$,

$$
(k=1,2, \ldots, n)
$$

MCGM: $\quad \hat{y}=\hat{x}^{(0)}(k+1)\left(1+\frac{6.24176+6.84404}{2} \%\right)$

- Mathematical Model for Region II

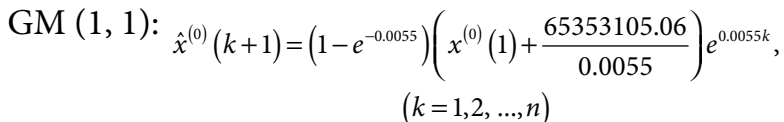

MCGM: $\quad \hat{y}=\hat{x}^{(0)}(k+1)\left(1+\frac{9.67+10.55}{2} \%\right)$

- Mathematical Model for Region III

$\operatorname{GM}(1,1): \hat{x}^{(0)}(k+1)=\left(1-e^{-0.0046}\right)\left(x^{(0)}(1)+\frac{359272055.97}{0.0046}\right) e^{0.0046 k}$, $(k=1,2, \ldots, n)$

MCGM:

$$
\hat{y}=\hat{x}^{(0)}(k+1)\left(1+\frac{11.4402+12.1185}{2} \%\right)
$$

- Mathematical Model for Region IV-A

$\mathrm{GM}(1,1): \hat{x}^{(0)}(k+1)=\left(1-e^{-0.0047}\right)\left(x^{(0)}(1)+\frac{93526613.77}{0.0047}\right) e^{0.0047 k}$,

$$
(k=1,2, \ldots, n)
$$

MCGM:

$$
\hat{y}=\hat{x}^{(0)}(k+1)\left(1+\frac{9.7674+10.2885}{2} \%\right)
$$


- Mathematical Model for Region IV-B

$\mathrm{GM}(1,1): \hat{x}^{(0)}(k+1)=\left(1-e^{-0.0070}\right)\left(x^{(0)}(1)+\frac{39468039.76}{0.0070}\right) e^{0.0070 k}$,

$$
(k=1,2, \ldots, n)
$$

MCGM: $\quad \hat{y}=\hat{x}^{(0)}(k+1)\left(1+\frac{11.3305+11.8766}{2} \%\right)$

- Mathematical Model for Region V

$\mathrm{GM}(1,1){ }_{\hat{x}^{(0)}}(k+1)=\left(1-e^{-0.0055}\right)\left(x^{(0)}(1)+\frac{74173640.36}{0.0055}\right) e^{0.0055 k}$,

$$
(k=1,2, \ldots, n)
$$

MCGM:

$$
\hat{y}=\hat{x}^{(0)}(k+1)\left(1+\frac{10.2145+11.6700}{2} \%\right)
$$

- Mathematical Model for Region VI

GM $(1,1): \hat{x}^{(0)}(k+1)=\left(1-e^{-0.0064}\right)\left(x^{(0)}(1)+\frac{167813119.16}{0.0064}\right) e^{0.0064 k}$,

$$
(k=1,2, \ldots, n)
$$

MCGM:

$$
\hat{y}=\hat{x}^{(0)}(k+1)\left(1+\frac{10.2428+10.8593}{2} \%\right)
$$

- Mathematical Model for Region VII

$\mathrm{GM}(1,1): \hat{x}^{(0)}(k+1)=\left(1-e^{-0.0039}\right)\left(x^{(0)}(1)+\frac{327277575.19}{0.0039}\right) e^{0.0039 k}$,

$$
(k=1,2, \ldots, n)
$$

MCGM:

$$
\hat{y}=\hat{x}^{(0)}(k+1)\left(1+\frac{3.0344+3.5324}{2} \%\right)
$$

- Mathematical Model for Region VIII

$\mathrm{GM}(1,1): \hat{x}^{(0)}(k+1)=\left(1-e^{-0.0053}\right)\left(x^{(0)}(1)+\frac{54298015.27}{0.0053}\right) e^{0.0053 k}$,

$$
(k=1,2, \ldots, n)
$$

MCGM: $\quad \hat{y}=\hat{x}^{(0)}(k+1)\left(1+\frac{9.8056+10.6602}{2} \%\right)$

- Mathematical Model for Region IX

$$
\begin{aligned}
& \operatorname{GM}(1,1): \hat{x}^{(0)}(k+1)=\left(1-e^{-0.0045}\right)\left(x^{(0)}(1)+\frac{67007156.6662}{0.004}\right) e^{0.004 k}, \\
& (k=1,2, \ldots, n)
\end{aligned}
$$

MCGM: $\quad \hat{y}=\hat{x}^{(0)}(k+1)\left(1+\frac{2.0884+2.4682}{2} \%\right)$

- Mathematical Model for Region X

$\mathrm{GM}(1,1): \hat{x}^{(0)}(k+1)=\left(1-e^{-0.0022}\right)\left(x^{(0)}(1)+\frac{161096979.11}{0.0022}\right) e^{0.0022 k}$, $(k=1,2, \ldots, n)$

MCGM: $\quad \hat{y}=\hat{x}^{(0)}(k+1)\left(1+\frac{-0.4341+0.1678}{2} \%\right)$

- Mathematical Model for Region XI

$\mathrm{GM}(1,1): \hat{x}^{(0)}(k+1)=\left(1-e^{-0.0029}\right)\left(x^{(0)}(1)+\frac{180969303.57}{0.0029}\right) e^{0.00292 k}$, $(k=1,2, \ldots, n)$

MCGM: $\quad \hat{y}=\hat{x}^{(0)}(k+1)\left(1+\frac{6.6928+7.1215}{2} \%\right)$

- Mathematical Model for Region XII

$\mathrm{GM}(1,1): \hat{x}^{(0)}(k+1)=\left(1-e^{-0.0038}\right)\left(x^{(0)}(1)+\frac{102908578.74}{0.0038}\right) e^{0.00389 k}$, $(k=1,2, \ldots, n)$

MCGM:

$$
\hat{y}=\hat{x}^{(0)}(k+1)\left(1+\frac{8.5888+8.9914}{2} \%\right)
$$

- Mathematical Model for CARAGA

GM $(1,1): \hat{x}^{(0)}(k+1)=\left(1-e^{-0.0050}\right)\left(x^{(0)}(1)+\frac{53418651.68}{0.0049}\right) e^{0.0049 k}$,

$$
(k=1,2, \ldots, n)
$$

MCGM: $\quad \hat{y}=\hat{x}^{(0)}(k+1)\left(1-\frac{3.1825+2.7348}{2} \%\right)$

- Mathematical Model for ARMM

$\mathrm{GM}(1,1): \hat{x}^{(0)}(k+1)=\left(1-e^{-0.0034}\right)\left(x^{(0)}(1)+\frac{13275822.76}{0.0034}\right) e^{0.0034 k}$,

$$
(k=1,2, \ldots, n)
$$

MCGM: $\quad \hat{y}=\hat{x}^{(0)}(k+1)\left(1-\frac{4.4158+3.7429}{2} \%\right)$

- Mathematical Model for NCR 
$\mathrm{GM}(1,1): \hat{x}^{(0)}(k+1)=\left(1-e^{-0.0046}\right)\left(x^{(0)}(1)+\frac{359272055.97}{0.0046}\right) e^{0.0046 k}$, $(k=1,2, \ldots, n)$

MCGM:

$$
\hat{y}=\hat{x}^{(0)}(k+1)\left(1+\frac{11.4402+12.1185}{2} \%\right)
$$

\subsubsection{Comparison of $G M(1,1)$ and Markov Models}

According to Table 7, the value of RMSE, MSE, MAE, and NRMSE of $\operatorname{GM}(1,1)$ is higher than the Markov's. Based on the MAPE of the two models, the value of Markov is nearly zero. Hence, a good forecast of values is obtained. From the information from the table above, the prediction from the Markov model is better than the prediction of the GM $(1,1)$ model.

\subsection{The Actual Data and the Forecasted Data}

The Figures of graphs contain blue lines that represent the actual data and red lines which represent the predicted values of Markov-Chain Grey Model for the year 2018 to
2021. The tables below contain the predicted values of the MCGM in KWH for the year 2018 to 2021.

\subsubsection{NCR}

Based on Figure 19, we can see that the predicted values of NCR from year 2018 to 2021 increases compared to the actual value where the monthly electricity consumption from the year 2013 to 2018 fluctuates (see Table 8).

\subsubsection{Region I}

Based on Figure 20, we can see that the predicted values of Region I from year 2018 to 2021 increases compared to the actual value where the monthly electricity consumption from the year 2013 to 2018 fluctuates (see Table 9).

\subsubsection{CAR}

Based on Figure 21, we can see that the predicted values of CAR from year 2018 to 2021 increases compared to the actual value where the monthly electricity consumption from the year 2013 to 2018 fluctuates. The predicted values of the MCGM for January 2018 to December 2020 graphs like a line because the state used for predicting these values is constant (see Table 10).

Table 7. Forecasting errors between GM $(1,1)$ and Markov-Chain Grey Model

\begin{tabular}{|l|l|l|l|l|l|}
\hline Model & (RMSE) & (MSE) & (MAPE) & (MAE) & (NMSE) \\
\hline GM $(\mathbf{1}, \mathbf{1})$ & 40284032.632514 & 1622803285137450 & 8.352002 & 33974305.8325304 & 0.00918136719580996 \\
\hline MCGM & 5996193.61319247 & 35954337846890.2 & 1.073942 & 4227582.03151131 & 0.00020494214343992 \\
\hline
\end{tabular}

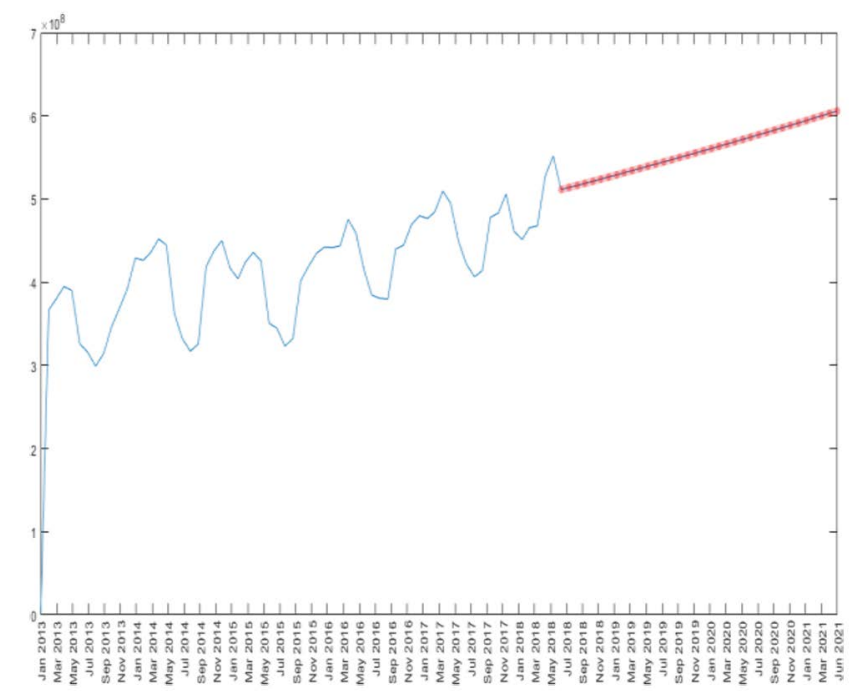

Figure 19. Graph of actual and predicted values of monthly electricity consumption for NCR. 
Table 8. Forecasted values of monthly electricity consumption for NCR

\begin{tabular}{|c|c|c|c|c|c|c|c|}
\hline \multirow{4}{*}{2018} & & January & February & March & April & May & June \\
\hline & MCGM & & & & & & \\
\hline & & July & August & September & October & November & December \\
\hline & MCGM & $511,370,824.10$ & $513,855,310.82$ & $516,351,868.37$ & $518,860,555.41$ & $521,381,430.86$ & $523,914,553.94$ \\
\hline \multirow{4}{*}{2019} & & January & February & March & April & May & June \\
\hline & MCGM & $526,459,984.16$ & $529,017,781.32$ & $531,588,005.48$ & $534,170,717.04$ & $536,765,976.66$ & $539,373,845.31$ \\
\hline & & July & August & September & October & November & December \\
\hline & MCGM & $541,994,384.24$ & $544,627,655.02$ & $547,273,719.50$ & $549,932,639.84$ & $552,604,478.50$ & $555,289,298.25$ \\
\hline \multirow{4}{*}{2020} & & January & February & March & April & May & June \\
\hline & MCGM & $557,987,162.15$ & $560,698,133.57$ & $563,422,276.21$ & $566,159,654.05$ & $568,910,331,39$ & $571,674,372.85$ \\
\hline & & July & August & September & October & November & December \\
\hline & MCGM & $574,451,843.37$ & $577,242,808.17$ & $580,047,332.84$ & $582,865,483.24$ & $585,6973,25.58$ & $588,542,926.38$ \\
\hline \multirow{2}{*}{2021} & & January & February & March & April & May & June \\
\hline & MCGM & $591,402,352.48$ & $594,275,671.06$ & $597,162,949.60$ & $600,064,255.95$ & $602,979,658.24$ & $605,909,224.97$ \\
\hline
\end{tabular}

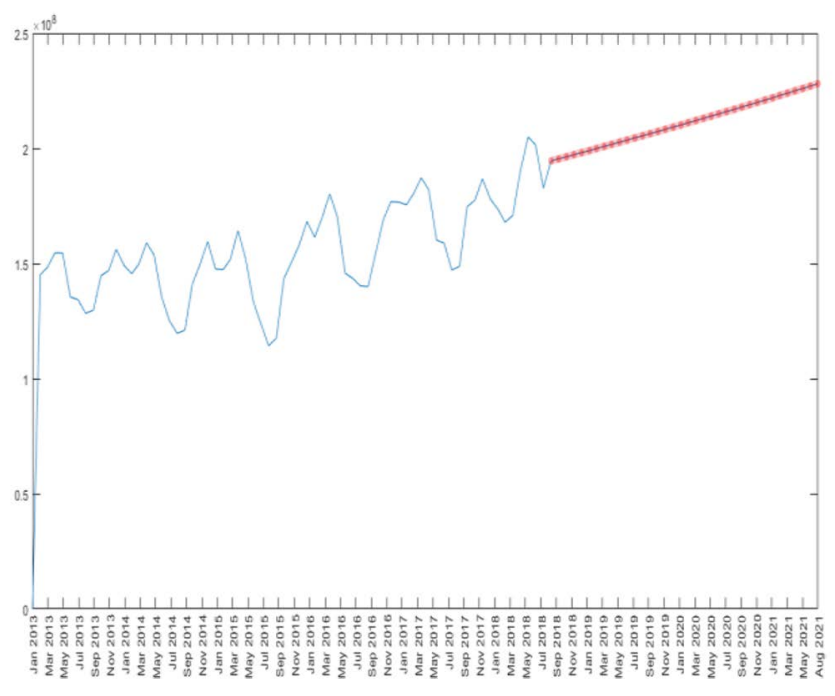

Figure 20. Graph of actual and predicted values of monthly electricity consumption for Region I.

Table 9. Forecasted values of monthly electricity consumption for Region I

\begin{tabular}{|l|l|l|l|l|l|l|l|}
\hline \multirow{3}{*}{2018} & & January & February & March & April & May & June \\
\cline { 2 - 8 } & MCGM & & & & & & \\
\cline { 2 - 8 } & & July & August & September & October & November & December \\
\cline { 2 - 8 } & MCGM & & & $194,608,816.90$ & $195,494,183.60$ & $196,383,578.24$ & $197,277,019.16$ \\
\hline \multirow{3}{*}{2019} & & January & February & March & April & May & June \\
\cline { 2 - 8 } & MCGM & $198,174,524.77$ & $199,076,113.54$ & $199,981,804.07$ & $200,891,615.00$ & $201,805,565.09$ & $202,723,573.17$ \\
\cline { 2 - 8 } & & July & August & September & October & November & December \\
\cline { 2 - 8 } & MCGM & $203,645,958.15$ & $204,572,439.04$ & $205,503,134.93$ & $206,438,064.99$ & $207,377,248.48$ & $208,320,704.75$ \\
\hline
\end{tabular}




\begin{tabular}{|l|l|l|l|l|l|l|l|}
\hline \multirow{3}{*}{2020} & & January & February & March & April & May & June \\
\cline { 2 - 8 } & MCGM & $209,268,453.26$ & $210,220,513.51$ & $211,176,905.13$ & $212,137,647.83$ & $213,102,761.39$ & $214,072,265.71$ \\
\cline { 2 - 8 } & & July & August & September & October & November & December \\
\cline { 2 - 8 } & MCGM & $215,046,180.76$ & $216,024,526.61$ & $217,007,323.40$ & $217,994,591.40$ & $218,986,350.95$ & $219,982,622.47$ \\
\cline { 2 - 8 } & & January & February & March & April & May & June \\
\cline { 2 - 8 } & & $220,983,426.50$ & $221,988,783.66$ & $222,998,714.66$ & $224,013,240.31$ & $225,032,381.51$ & $226,056,159.26$ \\
\cline { 2 - 7 } & MCGM & $227,084,594.65$ & $228,117,708.88$ & & October & November & December \\
\hline
\end{tabular}

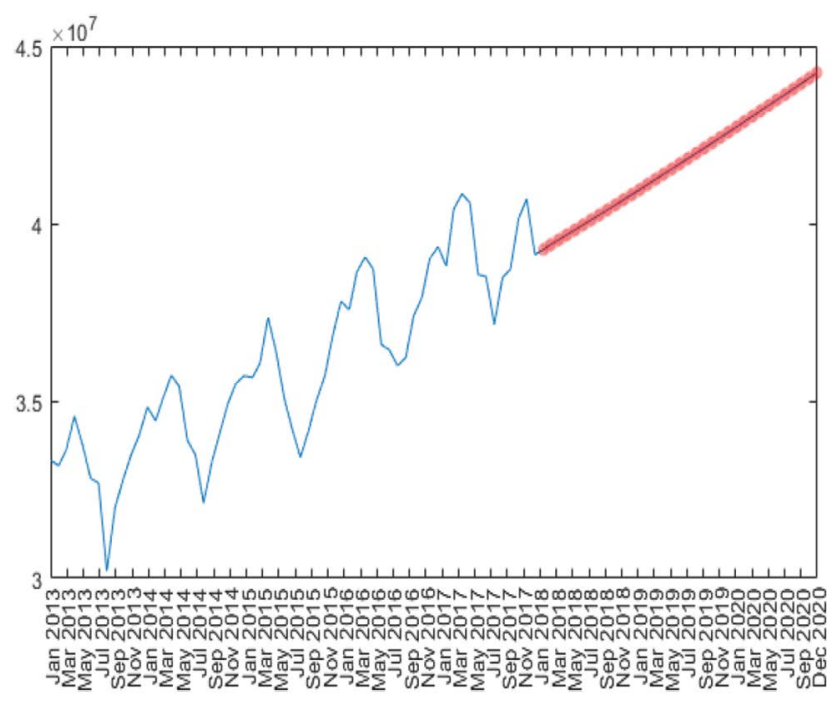

Figure 21. Graph of actual and predicted values of monthly electricity consumption for CAR.

Table 10. Forecasted values of monthly electricity consumption for CAR

\begin{tabular}{|c|c|c|c|c|c|c|c|}
\hline \multirow{4}{*}{2018} & & January & February & March & April & May & June \\
\hline & MCGM & $39,123,726.58$ & $39,261,768.63$ & $39,400,297.74$ & $39,539,315.63$ & $39,678,824.02$ & $39,818,824.64$ \\
\hline & & July & August & September & October & November & December \\
\hline & MCGM & $39,959,319.24$ & $40,100,309.55$ & $40,241,797.32$ & $40,383,784.31$ & $40,526,272.27$ & $40,669,262.99$ \\
\hline \multirow{4}{*}{2019} & & January & February & March & April & May & June \\
\hline & MCGM & $40,812,758.22$ & $40,956,759.76$ & $41,101,269.38$ & $41,246,288.89$ & $41,391,820.07$ & $41,537,864.74$ \\
\hline & & July & August & September & October & November & December \\
\hline & MCGM & $41,684,424.70$ & $41,831,501.78$ & $41,979,097.80$ & $42,127,214.58$ & $42,275,853.97$ & $42,425,017.82$ \\
\hline \multirow{4}{*}{2020} & & January & February & March & April & May & June \\
\hline & MCGM & $42,574,707.97$ & $42,724,925.27$ & $42,875,674.60$ & $43,026,954.82$ & $43,178,768.81$ & $43,331,118.45$ \\
\hline & & July & August & September & October & November & December \\
\hline & MCGM & $43,484,005.63$ & $43,637,432.25$ & $43,791,400.22$ & $43,945,911.43$ & $44,100,967.82$ & $44,256,571.30$ \\
\hline
\end{tabular}

\subsubsection{Region II}

Based on Figure 22, we can see that the predicted values of Region II from year 2018 to 2021 increases compared to the actual value where the monthly electricity consumption from the year 2013 to 2018 fluctuates. The forecasted values for the months of June in the year 2021 is lower than the peak of June 2018 (see Table 11).

\subsubsection{Region III}

Based on Figure 23, we can see that the predicted values of Region III from year 2018 to 2021 increases compared 


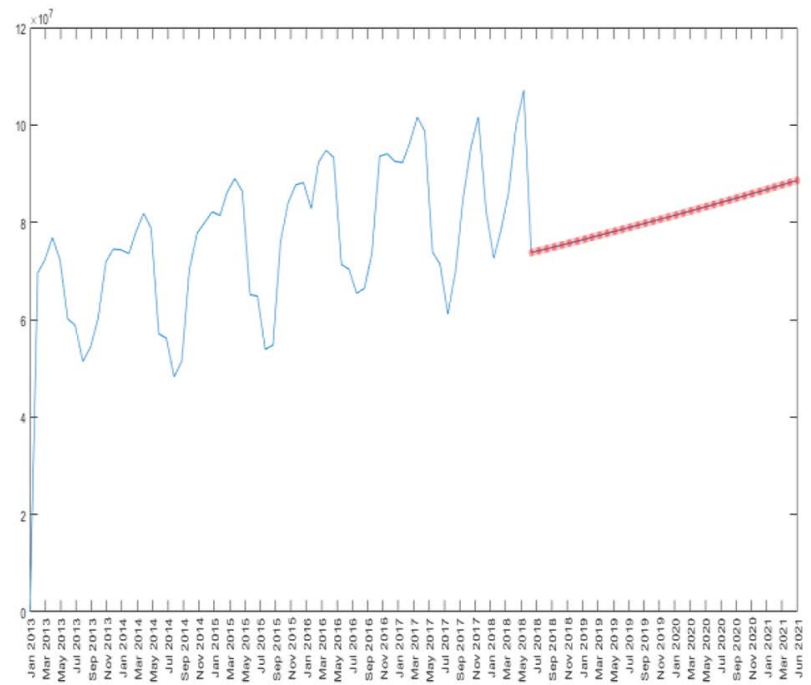

Figure 22. Graph of actual and predicted values of monthly electricity consumption for Region II.

Table 11. Forecasted values of monthly electricity consumption for Region II

\begin{tabular}{|c|c|c|c|c|c|c|c|}
\hline \multirow[t]{4}{*}{2018} & & January & February & March & April & May & June \\
\hline & MCGM & & & & & & \\
\hline & & July & August & September & October & November & December \\
\hline & MCGM & $73,777,433.98$ & $74,165,034.56$ & $74,554,671.45$ & $74,946,355.36$ & $75,340,097.04$ & $75,735,907.30$ \\
\hline \multirow[t]{4}{*}{2019} & & January & February & March & April & May & June \\
\hline & MCGM & $76,133,797.01$ & $76,533,777.09$ & $76,935,858.52$ & $77,340,052.35$ & $77,746,369.67$ & $78,154,821.63$ \\
\hline & & July & August & September & October & November & December \\
\hline & MCGM & $78,565,419.46$ & $78,978,174.42$ & $79,393,097.86$ & $79,810,201.15$ & $80,229,495.76$ & $80,650,993.19$ \\
\hline \multirow[t]{4}{*}{2020} & & January & February & March & April & May & June \\
\hline & MCGM & $81,074,705.02$ & $81,500,642.89$ & $81,928,818.48$ & $81,928,818.48$ & $82,791,929.93$ & $83,226,889.48$ \\
\hline & & July & August & September & October & November & December \\
\hline & MCGM & $83,664,134.17$ & $84,103,675.98$ & $84,545,526.98$ & $84,989,699.32$ & $85,436,205.18$ & $85,885,056.83$ \\
\hline \multirow[t]{2}{*}{2021} & & January & February & March & April & May & June \\
\hline & MCGM & $86,336,266.58$ & $86,789,846.83$ & $87,245,810.03$ & $87,704,168.70$ & $88,164,935.42$ & $88,628,122.85$ \\
\hline
\end{tabular}

to the actual value where the monthly electricity consumption from the year 2013 to 2018 fluctuates. The predicted values of the MCGM for July 2018 to June 2021 graphs like a line because the state used for predicting these values is constant (see Table 12).

\subsubsection{Region IV-A}

Based on Figure 24, we can see that the predicted values of Region IV-A from year 2018 to 2021 increases compared to the actual value where the monthly electricity consumption from the year 2013 to 2018 fluctuates. The predicted values of the MCGM for July 2018 to June 2021 graphs like a line because the state used for predicting these values is constant (see Table 13).

\subsubsection{Region $I V-B$}

Based on Figure 25, we can see that the predicted values of Region IV-B from year 2018 to 2021 increases compared to the actual value where the monthly electricity consumption from the year 2013 to 2018 fluctuates. The predicted values of the MCGM for January 2018 to December 2020 graphs like a line because the state used for predicting these values is constant (see Table 14). 


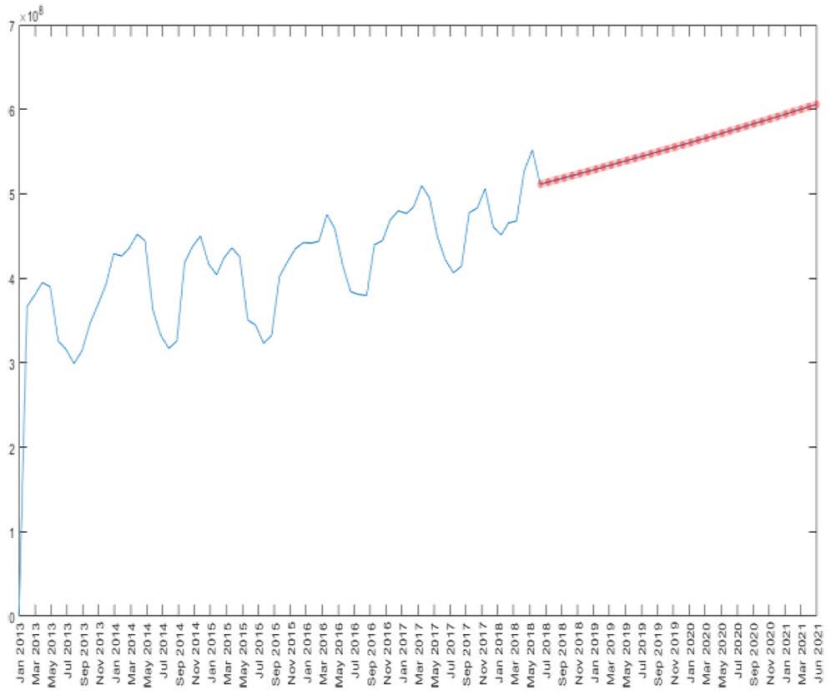

Figure 23. Graph of actual and predicted values of monthly electricity consumption for Region III.

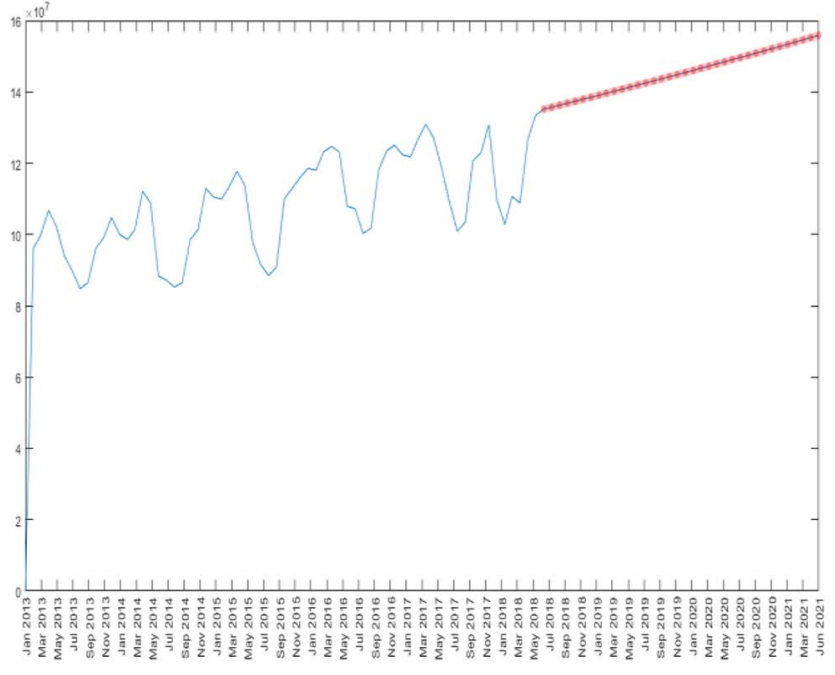

Figure 24. Graph of actual and predicted values of monthly electricity consumption for Region IV.

Table 12. Forecasted values of monthly electricity consumption for Region III

\begin{tabular}{|l|l|l|l|l|l|l|l|}
\hline \multirow{5}{*}{2018} & & January & February & March & April & May & June \\
\cline { 2 - 8 } & MCGM & & & & & & \\
& & July & August & September & October & November & December \\
\cline { 2 - 8 } & MCGM & $511,370,824.10$ & $513,855,310.82$ & $516,351,868.37$ & $518,860,555.41$ & $521,381,430.86$ & $523,914,553.94$ \\
\hline \multirow{5}{*}{2019} & & January & February & March & April & May & June \\
\cline { 2 - 8 } & MCGM & $526,459,984.16$ & $529,017,781.32$ & $531,588,005.48$ & $534,170,717.04$ & $536,765,976.66$ & $539,373,845.31$ \\
\cline { 2 - 8 } & & July & August & September & October & November & December \\
\cline { 2 - 8 } & MCGM & $541,994,384.24$ & $544,627,655.02$ & $547,273,719.50$ & $549,932,639.84$ & $552,604,478.50$ & $555,289,298.25$ \\
\cline { 2 - 8 } & MCGM & $557,987,162.15$ & $560,698,133.57$ & $563,422,276.21$ & $566,159,654.05$ & $568,910,331.39$ & $571,674,372.85$ \\
\cline { 2 - 8 } & & July & August & September & October & November & December \\
\cline { 2 - 8 } & MCGM & $574,451,843.37$ & $577,242,808.17$ & $580,047,332.84$ & $582,865,483.24$ & $585,697,325.58$ & $588,542,926.38$ \\
\hline \multirow{3}{*}{2021} & & January & February & March & April & May & June \\
\cline { 2 - 8 } & MCGM & $591,402,352.48$ & $594,275,671.06$ & $597,162,949.60$ & $600,064,255.95$ & $602,979,658.24$ & $605,909,224.97$ \\
\hline
\end{tabular}

Table 13. Forecasted values of monthly electricity consumption for Region IV - A

\begin{tabular}{|c|c|c|c|c|c|c|c|}
\hline \multirow[t]{4}{*}{2018} & & January & February & March & April & May & June \\
\hline & MCGM & & & & & & \\
\hline & & July & August & September & October & November & December \\
\hline & MCGM & $135,136,607.98$ & $135,688,460.32$ & $136,242,566.25$ & $136,798,934.95$ & $137,357,575.68$ & $137,918,497.71$ \\
\hline \multirow[t]{4}{*}{2019} & & January & February & March & April & May & June \\
\hline & MCGM & $138,481,710.36$ & $139,047,222.97$ & $139,615,044.95$ & $140,185,185.72$ & $140,757,654.76$ & $141,332,461.56$ \\
\hline & & July & August & September & October & November & December \\
\hline & MCGM & $141,909,615.69$ & $142,489,126.71$ & $143,071,004.26$ & $143,655,258.01$ & $144,241,897.65$ & $144,830,932.93$ \\
\hline
\end{tabular}




\begin{tabular}{|c|c|c|c|c|c|c|c|}
\hline 2020 & & January & February & March & April & May & June \\
\cline { 2 - 8 } & MCGM & $145,422,373.63$ & $146,016,229.57$ & $146,612,510.63$ & $147,211,226.69$ & $147,812,387.71$ & $148,416,003.66$ \\
\cline { 2 - 8 } & & July & August & September & October & November & December \\
\cline { 2 - 8 } & MCGM & $149,022,084.58$ & $149,630,640.53$ & $150,241,681.62$ & $150,855,218.00$ & $151,471,259.84$ & $152,089,817.40$ \\
\hline \multirow{2}{*}{2021} & & January & February & March & April & May & June \\
\cline { 2 - 8 } & MCGM & $152,710,900.94$ & $153,334,520.77$ & $153,960,687.26$ & $154,589,410.80$ & $155,220,701.83$ & $155,854,570.84$ \\
\hline
\end{tabular}

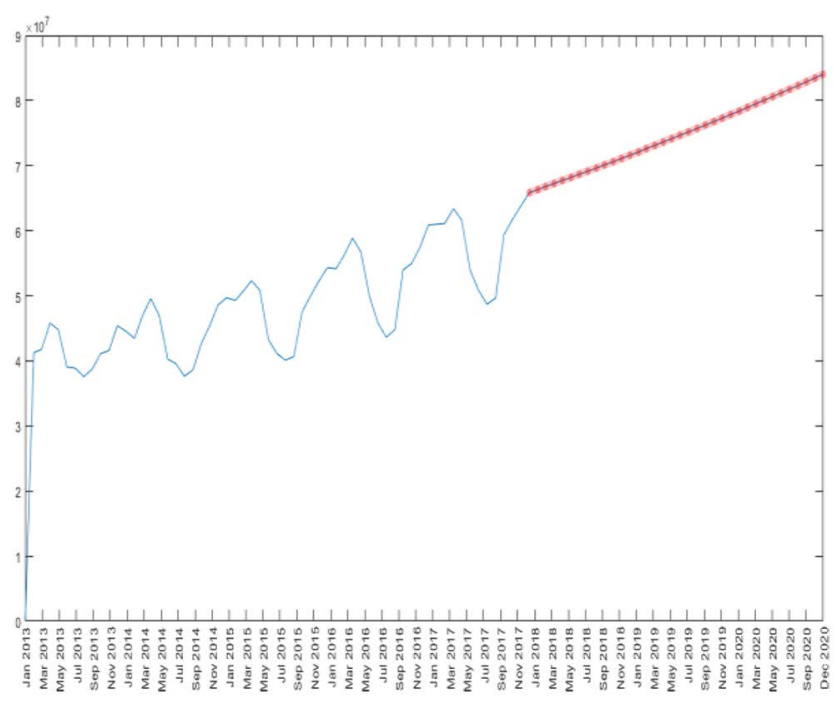

Figure 25. Graph of actual and predicted values of monthly electricity consumption for Region IV-B.

Table 14. Forecasted values of monthly electricity consumption for Region IV - B

\begin{tabular}{|c|c|c|c|c|c|c|c|}
\hline \multirow{4}{*}{2018} & & January & February & March & April & May & June \\
\hline & MCGM & $65,854,877.66$ & $66,315,433.47$ & $66,779,210.18$ & $67,246,230.30$ & $67,716,516.53$ & $68,190,091.71$ \\
\hline & & July & August & September & October & November & December \\
\hline & MCGM & $68,666,978.83$ & $69,147,201.06$ & $59,530,781.72$ & $70,117,744.30$ & $70,608,112.45$ & $71,101,909.99$ \\
\hline \multirow{4}{*}{2019} & & January & February & March & April & May & June \\
\hline & MCGM & $71,599,160.90$ & $72,099,889.33$ & $72,604,119.60$ & $73,111,876.21$ & $73,623,183.81$ & $74,138,067.23$ \\
\hline & & July & August & September & October & November & December \\
\hline & MCGM & $73,111,876.21$ & $73,623,183.81$ & $74,138,067.23$ & $74,656,551.50$ & $75,178,661.78$ & $75,704,423.43$ \\
\hline \multirow{4}{*}{2020} & & January & February & March & April & May & June \\
\hline & MCGM & $76,233,862.00$ & $76,767,003.19$ & $77,303,872.91$ & $77,844,497.22$ & $78,388,902.39$ & $78,937,114.85$ \\
\hline & & July & August & September & October & November & December \\
\hline & MCGM & $79,489,161.23$ & $80,045,068.34$ & $80,604,863.19$ & $81,168,572.97$ & $81,736,225.04$ & $82,307,846.99$ \\
\hline
\end{tabular}




\subsubsection{Region $V$}

Based on Figure 26, we can see that the predicted values of Region V from year 2018 to 2021 increases compared to the actual value where the monthly electricity consumption from the year 2013 to 2018 fluctuates. The predicted values of the MCGM for January 2018 to December 2020 graphs like a line because the state used for predicting these values is constant (see Table 15).

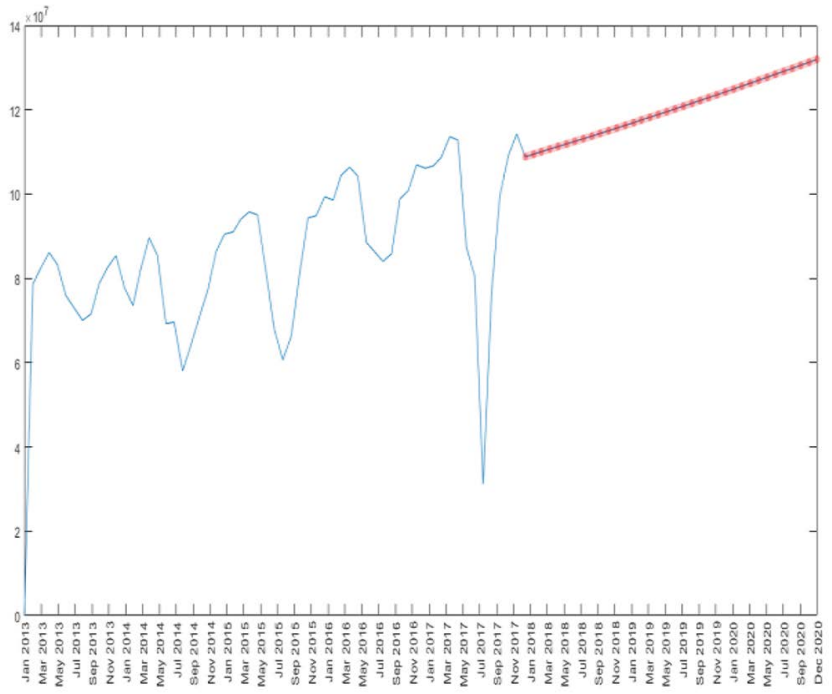

Figure 26. Graph of actual and predicted values of monthly electricity consumption for Region V.

\subsubsection{Region VI}

Based on Figure 27, we can see that the predicted values of Region VI from year 2018 to 2021 increases compared to the actual value where the monthly electricity consumption from the year 2013 to 2018 fluctuates. The predicted values of the MCGM for October 2018 to September 2021 graphs like a line because the state used for predicting these values is constant (see Table 16).

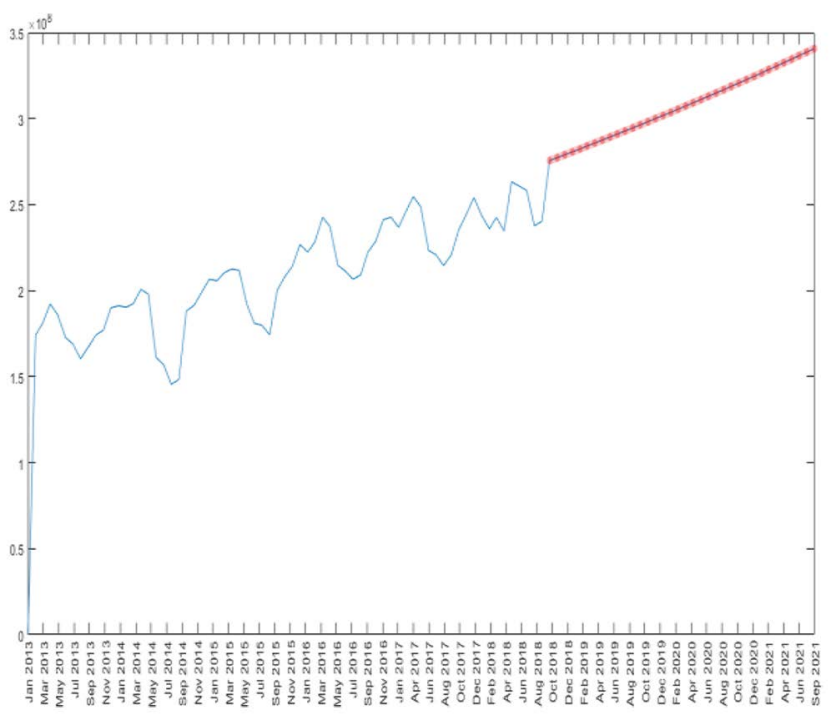

Figure 27. Graph of actual and predicted values of monthly electricity consumption for Region VI.

Table 15. Forecasted values of monthly electricity consumption for Region V

\begin{tabular}{|c|c|c|c|c|c|c|c|}
\hline \multirow{5}{*}{2018} & & January & February & March & April & May & June \\
\cline { 2 - 8 } & MCGM & $108,851,920.13$ & $109,453,030.68$ & $110,057,460.74$ & $110,665,228.63$ & $111,276,352.78$ & $111,890,851.73$ \\
\cline { 2 - 8 } & & July & August & September & October & November & December \\
\cline { 2 - 9 } & MCGM & $112,508,744.11$ & $113,130,048.66$ & $113,754,784.23$ & $114,382,969.77$ & $115,014,624.32$ & $115,649,767.04$ \\
\cline { 2 - 9 } & & January & February & March & April & May & June \\
\cline { 2 - 9 } & MCGM & $116,288,417.20$ & $116,930,594.16$ & $117,576,317.40$ & $118,225,606.50$ & $118,878,481.16$ & $119,534,961.17$ \\
\cline { 2 - 9 } & MCGM & $120,195,066.45$ & $120,858,817.01$ & $121,526,232.99$ & $122,197,334.63$ & $122,8,142.2172$ & $123,550,676.39$ \\
\hline \multirow{3}{*}{2020} & January & February & March & April & May & June \\
\cline { 2 - 9 } & MCGM & $124,232,957.57$ & $124,919,006.49$ & $125,608,843.96$ & $126,302,490.91$ & $126,999,968.37$ & $127,701,297.49$ \\
\cline { 2 - 8 } & & July & August & September & October & November & December \\
\cline { 2 - 8 } & MCGM & $128,406,499.55$ & $129,115,595.93$ & $129,828,608.13$ & $130,545,557.79$ & $131,266,466.64$ & $131,991,356.56$ \\
\hline
\end{tabular}


Table 16. Forecasted values of monthly electricity consumption for Region VI

\begin{tabular}{|c|c|c|c|c|c|c|c|}
\hline \multirow{2}{*}{2018} & & July & August & September & October & November & December \\
\hline & MCGM & & & & $275,605,757.23$ & $277,279,945.64$ & $278,964,304.03$ \\
\hline \multirow{4}{*}{2019} & & January & February & March & April & May & June \\
\hline & MCGM & $437,750,902.62$ & $438,956,327.46$ & $440,165,071.66$ & $441,377,144.35$ & $442,592,554.69$ & $443,811,311.88$ \\
\hline & & July & August & September & October & November & December \\
\hline & MCGM & $445,033,425.14$ & $446,258,903.71$ & $447,487,756.84$ & $448,719,993.84$ & $449,955,624.03$ & $451,194,656.74$ \\
\hline \multirow{4}{*}{2020} & & January & February & March & April & May & June \\
\hline & MCGM & $452,437,101.35$ & $453,682,967.25$ & $454,932,263.86$ & $456,185,000.64$ & $457,441,187.04$ & $458,700,832.58$ \\
\hline & & July & August & September & October & November & December \\
\hline & MCGM & $459,963,946.78$ & $461,230,539.19$ & $462,500,619.38$ & $463,774,196.96$ & $465,051,281.57$ & $466,331,882.86$ \\
\hline \multirow{4}{*}{2021} & & January & February & March & April & May & June \\
\hline & MCGM & $467,616,010.51$ & $468,903,674.23$ & $470,194,883.76$ & $471,489,648.87$ & $472,787,979.34$ & $474,089,885.00$ \\
\hline & & July & August & September & October & November & December \\
\hline & MCGM & $475,395,375.68$ & $476,704,461.27$ & $478,017,151.66$ & & & \\
\hline
\end{tabular}

\subsubsection{Region VII}

Based on Figure 28, we can see that the predicted values of Region VII from year 2018 to 2021 increases compared to the actual value where the monthly electricity consumption from the year 2013 to 2018 fluctuates. The predicted values of the MCGM for October 2018 to September 2021 graphs like a line because the state used for predicting these values is constant (see Table 17).

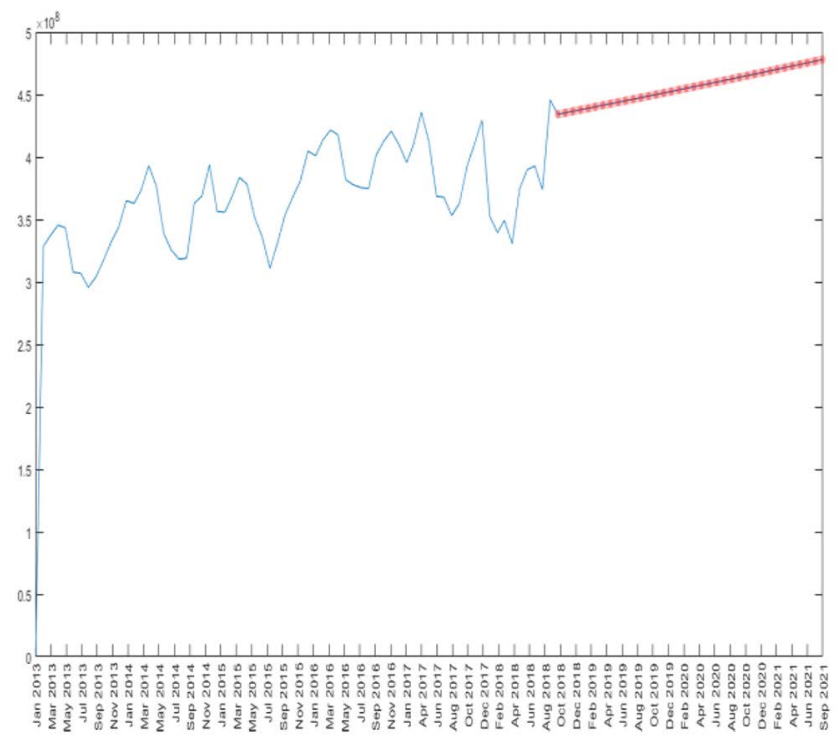

Figure 28. Graph of actual and predicted values of monthly electricity consumption for Region VII.

\subsubsection{Region VIII}

Based on Figure 29, we can see that the predicted values of Region VIII from year 2018 to 2021 increases compared to the actual value where the monthly electricity consumption from the year 2013 to 2018 fluctuates. The predicted values of the MCGM for October 2018 to September 2021 graphs like a line because the state used for predicting these values is constant (see Table 18).

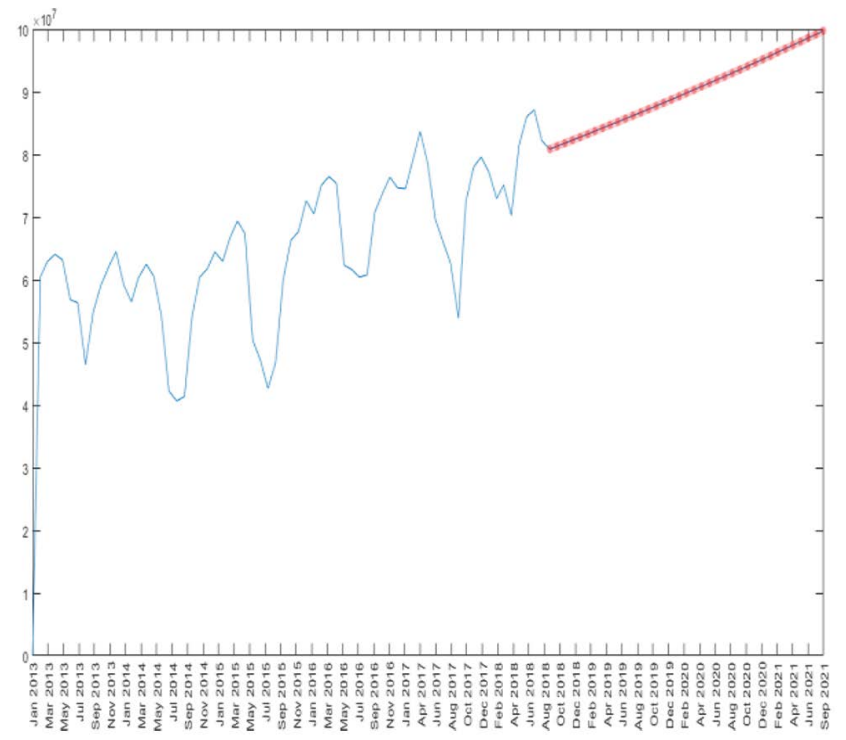

Figure 29. Graph of actual and predicted values of monthly electricity consumption for Region VIII. 
Table 17. Forecasted values of monthly electricity consumption for Region VII

\begin{tabular}{|l|l|l|l|l|l|l|l|}
\hline 2018 & & July & August & September & October & November & December \\
\cline { 2 - 8 } & MCGM & & & & $434,154,453.16$ & $435,349,974.55$ & $436,548,788.01$ \\
\hline \multirow{4}{*}{2019} & & January & February & March & April & May & June \\
\cline { 2 - 8 } & MCGM & $437,750,902.62$ & $438,956,327.46$ & $440,165,071.66$ & $441,377,144.35$ & $442,592,554.69$ & $443,811,311.88$ \\
\cline { 2 - 8 } & & July & August & September & October & November & December \\
\cline { 2 - 8 } & MCGM & $445,033,425.14$ & $446,258,903.71$ & $447,487,756.84$ & $448,719,993.84$ & $449,955,624.03$ & $451,194,656.74$ \\
\hline \multirow{4}{*}{2020} & & January & February & March & April & May & June \\
\cline { 2 - 8 } & MCGM & $452,437,101.35$ & $453,682,967.25$ & $454,932,263.86$ & $456,185,000.64$ & $457,441,187.04$ & $458,700,832.58$ \\
\cline { 2 - 8 } & & July & August & September & October & November & December \\
\cline { 2 - 8 } & MCGM & $459,963,946.78$ & $461,230,539.19$ & $462,500,619.38$ & $463,774,196.96$ & $465,051,281.57$ & $466,331,882.86$ \\
\cline { 2 - 8 } & & January & February & March & April & May & June \\
\cline { 2 - 8 } & MCGM & $467,616,010.51$ & $468,903,674.23$ & $470,194,883.76$ & $471,489,648.87$ & $472,787,979.34$ & $474,089,885.00$ \\
\cline { 2 - 8 } & & July & August & September & October & November & December \\
\cline { 2 - 8 } & MCGM & $475,395,375.68$ & $476,704,461.27$ & $478,017,151.66$ & & & \\
\hline
\end{tabular}

Table 18. Forecasted values of monthly electricity consumption for Region VIII

\begin{tabular}{|c|c|c|c|c|c|c|c|}
\hline \multirow{2}{*}{2018} & & July & August & September & October & November & December \\
\hline & MCGM & & & & $81,325,352.84$ & $81,801,150.20$ & $82,279,731.24$ \\
\hline \multirow{4}{*}{2019} & & January & February & March & April & May & June \\
\hline & MCGM & $82,761,112.23$ & $83,245,309.57$ & $83,732,339.72$ & $84,222,219.26$ & $84,714,964.86$ & $85,210,593.29$ \\
\hline & & July & August & September & October & November & December \\
\hline & MCGM & $85,709,121.42$ & $86,210,566.20$ & $86,714,944.71$ & $87,222,274.11$ & $87,732,571.65$ & $88,245,854.72$ \\
\hline \multirow{4}{*}{2020} & & January & February & March & April & May & June \\
\hline & MCGM & $88,762,140.77$ & $89,281,447.37$ & $89,803,792.20$ & $90,329,193.02$ & $90,857,667.73$ & $91,389,234.30$ \\
\hline & & July & August & September & October & November & December \\
\hline & MCGM & $91,923,910.82$ & $92,461,715.49$ & $93,002,666.61$ & $93,546,782.58$ & $94,094,081.93$ & $94,644,583.28$ \\
\hline \multirow{4}{*}{2021} & & January & February & March & April & May & June \\
\hline & MCGM & $95,198,305.36$ & $95,755,267.01$ & $96,315,487.18$ & $96,878,984.95$ & $97,445,779.49$ & $98,015,890.08$ \\
\hline & & July & August & September & October & November & December \\
\hline & MCGM & $98,589,336.13$ & $99,166,137.15$ & $99,746,312.76$ & & & \\
\hline
\end{tabular}

\subsubsection{Region IX}

Based on Figure 30, we can see that the predicted values of Region IX from year 2018 to 2021 increases compared to the actual value where the monthly electricity consumption from the year 2013 to 2018 fluctuates. The predicted values of the MCGM for September 2018 to August 2021 graphs like a line because the state used for predicting these values is constant (see Table 19).

\subsubsection{Region $X$}

Based on the Figure 31, we can see that the predicted values of Region X from year 2018 to 2021 increases compared to the actual value where the monthly electricity consumption from the year 2013 to 2018 fluctuates. The predicted values of the MCGM for January 2019 to December 2021 graphs like a line because the state used for predicting these values is constant (see Table 20). 


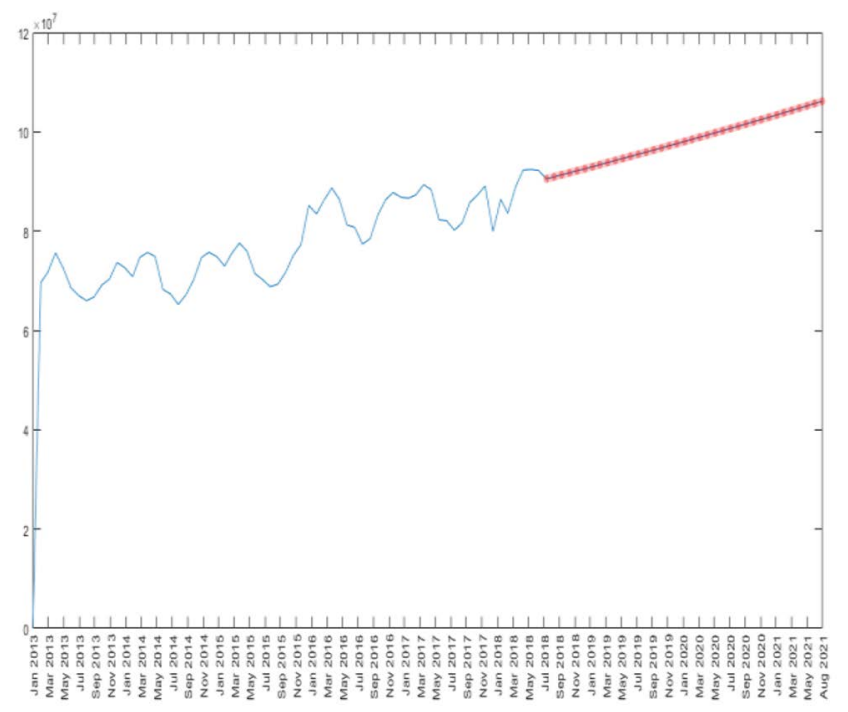

Figure 30. Graph of actual and predicted values of monthly electricity consumption for Region IX.

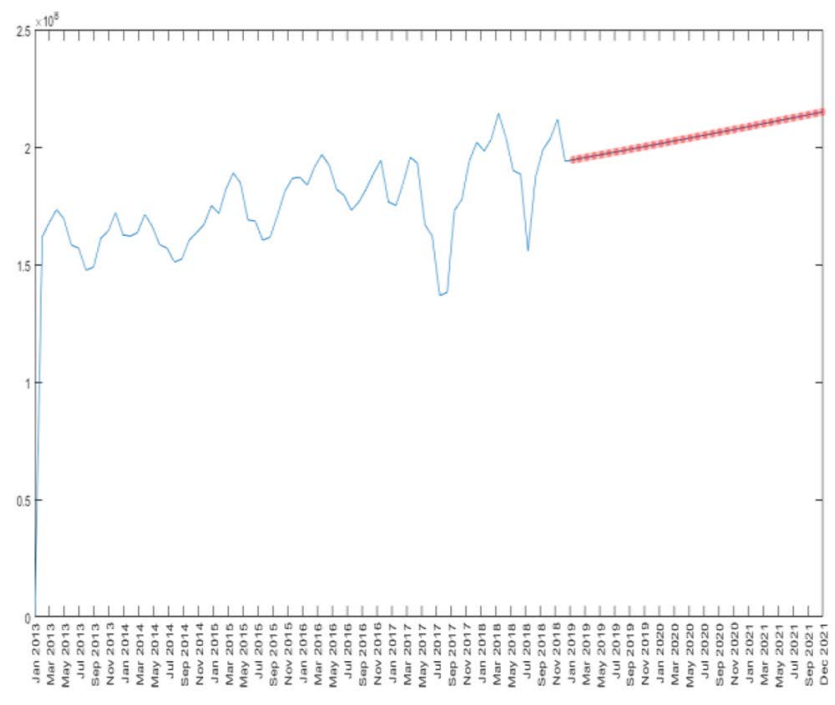

Figure 31. Graph of actual and predicted values of monthly electricity consumption for Region X.

Table 19. Forecasted values of monthly electricity consumption for Region IX

\begin{tabular}{|c|c|c|c|c|c|c|c|}
\hline \multirow{2}{*}{2018} & & July & August & September & October & November & December \\
\hline & MCGM & & & $90,944,059.50$ & $91,347,498.76$ & $91,752,721.74$ & $92,159,754.36$ \\
\hline \multirow{4}{*}{2019} & & January & February & March & April & May & June \\
\hline & MCGM & $92,568,586.60$ & $92,979,232.47$ & $93,391,700.02$ & $93,805,997.32$ & $94,222,132.50$ & $94,640,113.71$ \\
\hline & & July & August & September & October & November & December \\
\hline & MCGM & $95,059,949.14$ & $95,481,647.01$ & $95,905,215.58$ & $97,187,228.70$ & $97,187,228.70$ & $97,187,228.70$ \\
\hline \multirow{4}{*}{2020} & & January & February & March & April & May & June \\
\hline & MCGM & $97,618,363.45$ & $98,051,410.76$ & $98,486,379.13$ & $98,923,277.07$ & $99,362,113.15$ & $99,802,895.96$ \\
\hline & & July & August & September & October & November & December \\
\hline & MCGM & $100,245,634.13$ & $100,690,336.35$ & $101,137,011.32$ & $101,585,667.80$ & $102,036,314.58$ & $102,488,960.48$ \\
\hline \multirow{4}{*}{2021} & & January & February & March & April & May & June \\
\hline & MCGM & $102,943,614.38$ & $103,400,285.17$ & $103,858,981.82$ & $104,319,713.30$ & $104,782,488.65$ & $105,247,316.92$ \\
\hline & & July & August & September & October & November & December \\
\hline & MCGM & $105,714,207.23$ & $106,183,168.73$ & & & & \\
\hline
\end{tabular}

Table 20. Forecasted values of monthly electricity consumption for Region X

\begin{tabular}{|c|c|c|c|c|c|c|c|}
\hline \multirow{4}{*}{2019} & & January & February & March & April & May & June \\
\cline { 2 - 8 } & MCGM & $193,941,169.66$ & $194,512,857.96$ & $195,086,231.46$ & $195,661,295.10$ & $196,238,053.89$ & $196,816,512.81$ \\
\cline { 2 - 8 } & & July & August & September & October & November & December \\
\cline { 2 - 8 } & MCGM & $197,396,676.88$ & $197,978,551.13$ & $198,562,140.58$ & $199,147,450.31$ & $199,734,485.38$ & $200,323,250.88$ \\
\hline \multirow{3}{*}{2020} & & January & February & March & April & May & June \\
\cline { 2 - 9 } & MCGM & $200,913,751.90$ & $201,505,993.57$ & $202,099,981.01$ & $202,695,719.38$ & $203,293,213.82$ & $203,892,469.53$ \\
\cline { 2 - 9 } & & July & August & September & October & November & December \\
\cline { 2 - 9 } & MCGM & $204,493,491.68$ & $205,096,285.50$ & $205,700,856.19$ & $206,307,209.00$ & $206,915,349.18$ & $207,525,282.01$ \\
\hline
\end{tabular}




\begin{tabular}{|c|c|c|c|c|c|c|c|}
\hline & & January & February & March & April & May & June \\
\cline { 2 - 8 } 2021 & MCGM & $208,137,012.75$ & $208,750,546.72$ & $209,365,889.23$ & $209,983,045.61$ & $210,602,021.20$ & $211,222,821.38$ \\
\cline { 2 - 8 } & & July & August & September & October & November & December \\
\cline { 2 - 8 } & \multirow{2}{*}{ MCGM } & $211,845,451.51$ & $212,469,917.00$ & $213,096,223.24$ & $213,724,375.68$ & $214,354,379.75$ & $214,986,240.90$ \\
\hline
\end{tabular}

\subsubsection{Region XI}

Based on Figure 32, we can see that the predicted values of Region XI from year 2018 to 2021 increases compared to the actual value where the monthly electricity consumption from the year 2013 to 2018 fluctuates. The predicted values of the MCGM for January 2019 to December 2021 graphs like a line because the state used for predicting these values is constant (see Table 21).

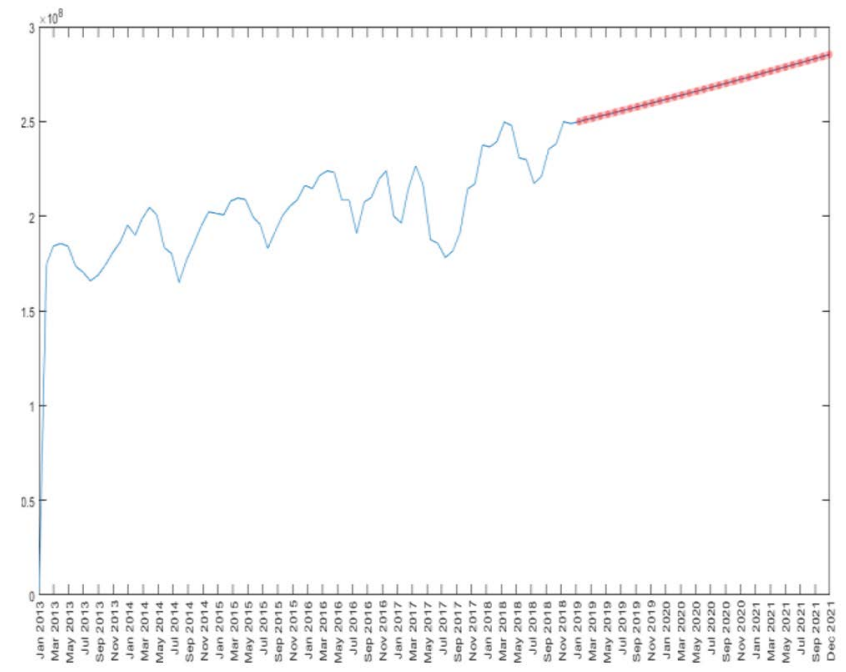

Figure 32. Graph of actual and predicted values of monthly electricity consumption for Region XI.

\subsubsection{Region XII}

Based on Figure 33, we can see that the predicted values of Region XII from year 2018 to 2021 increases compared to the actual value where the monthly electricity consumption from the year 2013 to 2018 fluctuates. The predicted values of the MCGM for January 2019 to December 2021 graphs like a line because the state used for predicting these values is constant (see Table 22).

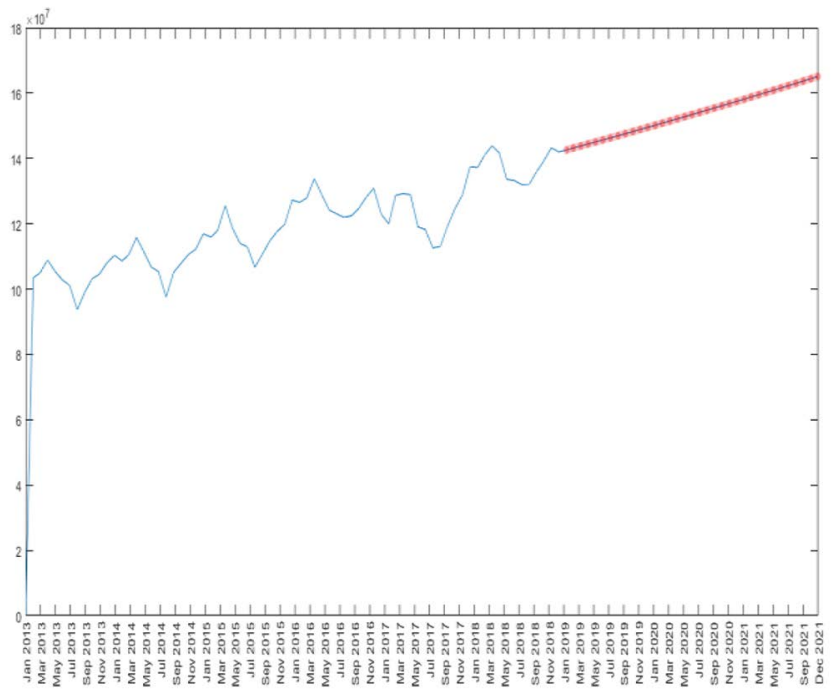

Figure 33. Graph of actual and predicted values of monthly electricity consumption for REGION XII.

Table 21. Forecasted values of monthly electricity consumption for Region XI

\begin{tabular}{|c|c|c|c|c|c|c|c|}
\hline \multirow{4}{*}{2019} & & January & February & March & April & May & June \\
\hline & MCGM & $248,861,569.45$ & $249,835,876.40$ & $250,813,997.82$ & $251,795,948.64$ & $252,781,743.86$ & $253,771,398.51$ \\
\hline & & July & August & September & October & November & December \\
\hline & MCGM & $254,764,927.72$ & $255,762,346.66$ & $256,763,670.54$ & $257,768,914.66$ & $258,778,094.37$ & $259,791,225.08$ \\
\hline \multirow{4}{*}{2020} & & January & February & March & April & May & June \\
\hline & MCGM & $260,808,322.24$ & $261,829,401.40$ & $262,854,478.15$ & $263,883,568.12$ & $264,916,687.04$ & $265,953,850.68$ \\
\hline & & July & August & September & October & November & December \\
\hline & MCGM & $266,995,074.87$ & $268,040,375.51$ & $269,089,768.56$ & $270,143,270.05$ & $271,200,896.05$ & $272,252,552.72$ \\
\hline \multirow{4}{*}{2021} & & January & February & March & April & May & June \\
\hline & MCGM & $273,328,586.26$ & $274,398,682.95$ & $215,472,959.14$ & $276,551,461.21$ & $277,634,175.64$ & $218,721,128.95$ \\
\hline & & July & August & September & October & November & December \\
\hline & MCGM & $279,812,337.75$ & $280,907,818.70$ & $282,007,588.51$ & $283,111,663.98$ & $284,220,061.97$ & $285,332,799.40$ \\
\hline
\end{tabular}


Table 22. Forecasted values of monthly electricity consumption for Region XII

\begin{tabular}{|c|c|c|c|c|c|c|c|}
\hline \multirow{4}{*}{2019} & & January & February & March & April & May & June \\
\hline & MCGM & $141,957,151.22$ & $142,570,989.16$ & $143,187,481.41$ & $143,806,639.43$ & $144,428,474.75$ & $145,052,998.96$ \\
\hline & & July & August & September & October & November & December \\
\hline & MCGM & $145,680,223.68$ & $146,310,160.59$ & $146,942,821.41$ & $147,578,217.92$ & $148,216,361.96$ & $148,857,265.40$ \\
\hline \multirow{4}{*}{2020} & & January & February & March & April & May & June \\
\hline & MCGM & $149,500,940.18$ & $150,147,398.27$ & $150,795,551.72$ & $151,448,712.61$ & $152,103,593.08$ & $152,761,305.32$ \\
\hline & & July & August & September & October & November & December \\
\hline & MCGM & $153,421,861.58$ & $154,085,274.16$ & $154,751,555.41$ & $155,420,111.72$ & $156,092,773.57$ & $156,767,735.46$ \\
\hline \multirow{4}{*}{2021} & & January & February & March & April & May & June \\
\hline & MCGM & $157,445,615.95$ & $158,126,427.68$ & $158,810,183.30$ & $159,496,895.56$ & $160,186,577.24$ & $160,879,241.17$ \\
\hline & & July & August & September & October & November & December \\
\hline & MCGM & $161,574,900.26$ & $162,273,567.45$ & $162,975,255.76$ & $163,679,978.24$ & $164,387,748.01$ & $165,098,578.26$ \\
\hline
\end{tabular}

\subsubsection{CARAGA}

Based on Figure 34, we can see that the predicted values of CARAGA from year 2018 to 2021 increases compared to the actual value where the monthly electricity consumption from the year 2013 to 2018 fluctuates. The predicted values of the MCGM for April 2018 to March 2021 graphs like a line because the state used for predicting these values is constant (see Table 23).

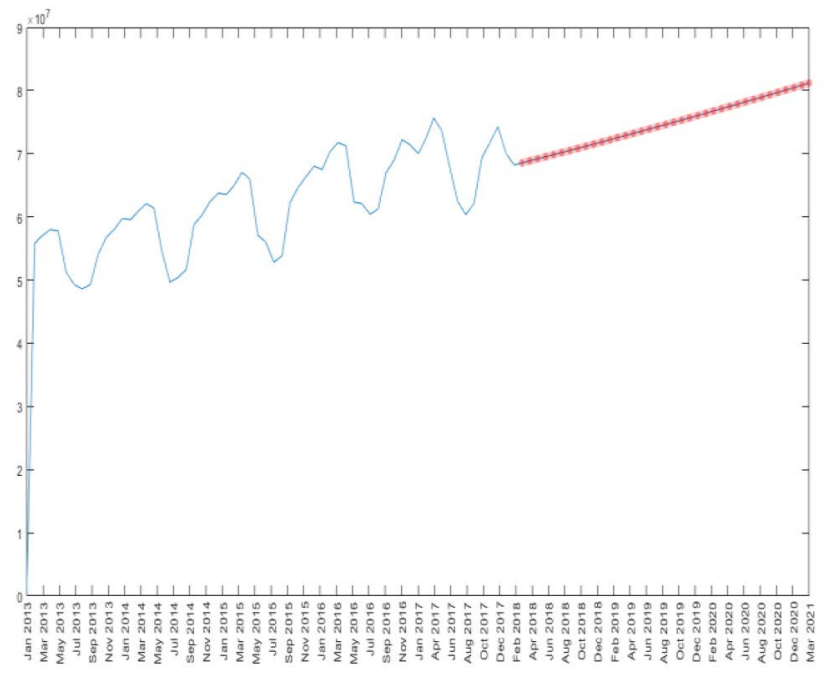

Figure 34. Graph of actual and predicted values of monthly electricity consumption for CARAGA

\subsubsection{ARMM}

Based on Figure 35, we can see that the predicted values of ARMM from year 2018 to 2021 increases compared to the actual value where the monthly electricity consumption from the year 2013 to 2018 fluctuates. The predicted values of the MCGM for January 2018 to December 2020 graphs like a line because the state used for predicting these values is constant (see Table 24).

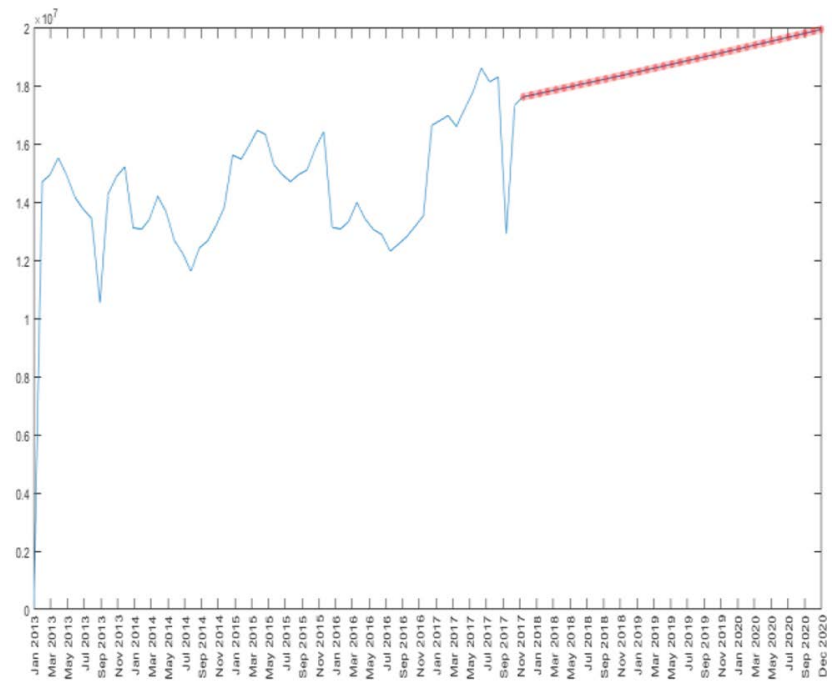

Figure 35. Graph of actual and predicted values of monthly electricity consumption for ARMM. 
Table 23. Forecasted values of monthly electricity consumption for CARAGA

\begin{tabular}{|c|c|c|c|c|c|c|c|}
\hline \multirow{4}{*}{2018} & & January & February & March & April & May & June \\
\hline & MCGM & & & & $68,869,706.00$ & $69,195,192.39$ & $69,522,217.08$ \\
\hline & & July & August & September & October & November & December \\
\hline & MCGM & $69,850,787.31$ & $70,180,910.41$ & $70,512,593.72$ & $70,845,844.59$ & $71,180,670.45$ & $71,517,078.74$ \\
\hline \multirow{4}{*}{2019} & & January & February & March & April & May & June \\
\hline & MCGM & $71,855,076.93$ & $72,194,6$ n.54 & $72,535,873.12$ & $72,878,686.25$ & $73,223,119.56$ & $73,569,180.70$ \\
\hline & & July & August & September & October & November & December \\
\hline & MCGM & $73,916,877.37$ & $74,266,217.30$ & $74,617,208.24$ & $74,969,858.01$ & $75,324,174.45$ & $75,680,165.42$ \\
\hline \multirow{4}{*}{2020} & & January & February & March & April & May & June \\
\hline & MCGM & $76,037,838.86$ & $76,397,202.70$ & $76,758,264.93$ & $77,121,033.59$ & $77,485,516.73$ & $77,851,722.47$ \\
\hline & & July & August & September & October & November & December \\
\hline & MCGM & $91,923,910.82$ & $92,461,715.49$ & $93,002,666.61$ & $93,546,782.58$ & $94,094,081.93$ & $94,644,583.28$ \\
\hline \multirow{4}{*}{2021} & & January & February & March & April & May & June \\
\hline & MCGM & $78,219,658.94$ & $78,589,334.32$ & $78,960,756.83$ & $79,333,934.72$ & $79,708,876.30$ & $80,085,589.90$ \\
\hline & & July & August & September & October & November & December \\
\hline & MCGM & $80,464,083.89$ & $80,844,366.69$ & $81,226,446.75$ & & & \\
\hline
\end{tabular}

Table 24. Forecasted values of monthly electricity consumption for ARMM

\begin{tabular}{|c|c|c|c|c|c|c|c|}
\hline \multirow{4}{*}{2018} & & January & February & March & April & May & June \\
\cline { 2 - 8 } & MCGM & $17,669,925.96$ & $17,730,847.51$ & $17,791,979.10$ & $17,853,321.46$ & $17,914,875.31$ & $17,976,641.38$ \\
\cline { 2 - 8 } & & July & August & September & October & November & December \\
\cline { 2 - 8 } & MCGM & $18,038,620.41$ & $18,100,813.12$ & $18,163,220.26$ & $18,225,842.57$ & $18,288,680.78$ & $18,351,735.64$ \\
\hline \multirow{4}{*}{2019} & & January & February & March & April & May & June \\
\cline { 2 - 8 } & MCGM & $18,415,007.90$ & $18,478,498.31$ & $18,542,207.62$ & $18,606,136.58$ & $18,670,285.95$ & $18,734,656.49$ \\
\cline { 2 - 8 } & & July & August & September & October & November & December \\
\cline { 2 - 8 } & MCGM & $18,799,248.97$ & $18,864,064.14$ & $18,929,102.78$ & $18,994,365.66$ & $19,059,853.55$ & $19,125,567.23$ \\
\hline \multirow{4}{*}{2020} & & January & February & March & April & May & June \\
\cline { 2 - 8 } & MCGM & $19,191,507.47$ & $19,257,675.05$ & $19,324,070.77$ & $19,390,695.40$ & $19,457,549.74$ & $19,524,634.57$ \\
\cline { 2 - 8 } & & July & August & September & October & November & December \\
\cline { 2 - 8 } & MCGM & $19,591,950.70$ & $19,659,498.91$ & $19,721,280.02$ & $19,795,294.81$ & $19,863,544.11$ & $19,932,028.71$ \\
\hline
\end{tabular}

\section{Conclusions}

This study is centered on forecasting the monthly electricity consumption for every region in the Philippines for the year 2018 up to 2021 using the current data of monthly electricity consumption of each region in the Philippines from 2013 up to 2018. Through the use of Markov-Chain Grey Model, we have seen that the behavior of the graph of the data is fluctuating - more often, the increase and decrease of the graph happen in the same month. Yet, the forecasted values increase linearly because of the states that affect the prediction. Furthermore, based on the computations of the forecasting errors, the MarkovChain Grey Model is a better model in forecasting than the GM $(1,1)$.

\section{Recommendations}

The researchers recommend to use forecasting models such as Autoregressive Integrated Moving Average (ARIMA) and Integrated Spatio-temporal and compare it 
to Markov-Chain Grey Markov model to assess for better accuracy.

\section{References}

1. Why is Electricity Important? 2019. https://www.enotes. com/homework-help/why-electricity-important-1116629

2. Philippine Power Statistics. 2018. https://www.doe.gov.ph/ philippine-power-statistics

3. Philippine Power Situation. 2015. https://www.doe.gov.ph/ electric-power/2015-philippine-power-situation

4. Philippine Power Situation Report. 2016. https://www. doe.gov.ph/electric-power/2016-philippine-power-situation-report

5. Electricity consumption across regions up by nearly $4 \%$ in 2017. 2018. https://www.bworldonline.com/electricity-consumption-up-by-nearly-4-in-2017/

6. Electric coops report stronger sales nationwide. 2018. https://business.inquirer.net/253145/electric-coops-report-stronger-sales-nationwide.

7. Hsu C, Wen Y. Improved grey prediction models for the trans-pacific air passenger market. Transportation Planning and Technology. 2007; 22(2):87-107. https://doi. org/10.1080/03081069808717622

8. Hsu C. Applying the grey prediction model to the global integrated circuit industry. Technological Forecasting and Social Change. 2003; 70(6):563-74. https://doi.org/10.1016/ S0040-1625(02)00195-6

9. He Y, Huang M. A Grey-Markov Forecasting Model for the electric power requirement in China. Mexican International Conference on Artificial Intelligence; 2005. p. 574-82. https://doi.org/10.1007/11579427_58

10. Huang $M, H e ~ Y$, Cen $H$. Predictive analysis on electric-power supply and demand in China. Renewable Energy. 2007; 32(7):1165-74. https://doi.org/10.1016/j. renene.2006.04.005

11. Li YZ, Luan R, Niu JC. Forecast of power generation for grid-connected photovoltaic system based on grey model and Markov chain. 2008 3rd IEEE Conference on Industrial Electronics and applications; 2008. p. 1729-33. PMCid: PMC3667673. https://doi.org/10.1109/ ICIEA.2008.4582816

12. Zhao W, Wang W, Wang Y, Zhao Y. Market share forecast of electricity in city residents' energy consumption based on Markov theory. 2008 Third International Conference on Electric Utility Deregulation and Restructuring and Power Technologies; 2008. p. 1-2.

13. Hsu YT, Liu MC, Yeh J, Hung HF. Forecasting the turning time of stock market based on Markov-Fourier grey model. Expert Systems with Applications. 2009; 36(4):8597-603. https://doi.org/10.1016/j.eswa.2008.10.075
14. Kumar U, Jain VK. Time series models (Grey-Markov, Grey Model with rolling mechanism and singular spectrum analysis) to forecast energy consumption in India. Energy. 2010; 35(4):1709-16. https://doi.org/10.1016/j. energy.2009.12.021

15. Prediction of rural electricity consumption based on Grey Markov model. 2011. https://ieeexplore.ieee.org/document/5986976

16. Sun CC. Improvement of renewable energy supply forecasts: The case of Taiwan renewable industry. African Journal of Business Management. 2013; 7(16):1436-44.

17. Kazemi A, Modarres M, Mehregan M. Energy demand forecasting of industrial sectors in Iran using a Markov Chain Grey Model. International Journal of Humanities. 2013; 20:1-12.

18. Zhao W, Wang J, Lu H. Combining forecasts of electricity consumption in China with time-varying weights updated by a high-order Markov Chain Model. Omega. 2014; 45:80-91. https://doi.org/10.1016/j.omega.2014.01.002

19. Yuan CQ, Yang YJ. Forecasting China's energy demand and self-sufficiency rate by grey forecasting model and Markov model. International Journal of Electrical Power and Energy Systems. 2015; 66:1-8. https://doi.org/10.1016/j. ijepes.2014.10.028

20. Analysis and modeling for China's electricity demand forecasting based on a New Mathematical Hybrid Method. 2017. https://www.researchgate.net/publication/314977360_ Analysis_and_Modeling_for_China's_Electricity_Demand_ Forecasting_Based_on_a_New_Mathematical_Hybrid_ Method

21. Xu N, Dang Y, Gong Y. Novel grey prediction model with nonlinear optimized time response method for forecasting of electricity consumption in China. Energy. 2017; 118:473-80. https://doi.org/10.1016/j.energy.2016.10.003

22. Wang ZX, Li Q, Pei LL. A seasonal GM $(1,1)$ model for forecasting the electricity consumption of the primary economic sectors. Energy. 2018; 154:522-34. https://doi. org/10.1016/j.energy.2018.04.155

23. Ismail Z. Forecasting gold prices using multiple linear regression method. American Journals of Applied Sciences. 2009; 6(8):1509-14. https://doi.org/10.3844/ ajassp.2009.1509.1514

24. Statistics How To: Mean Absolute Percentage error (MAPE). 2017. https://www.statisticshowto.datasciencecentral.com/ mean-absolute-percentage-error-mape/

25. Statistics How To: Absolute Error and Mean Absolute Error (MAE). 2016. https://www.statisticshowto.datasciencecentral.com/absolute-error

26. Statistics How To: Mean Squared Error: Definition and example. 2013. https://www.statisticshowto.datasciencecentral.com/mean-squared-error 
27. Statistics How To: RMSE: Root Mean Square Error. 2016. https://www.statisticshowto.datasciencecentral.com/rmse/

28. Normalised Mean Square Error. 2018. https://rem.jrc. ec.europa.eu/RemWeb/atmes2/20b.htm

29. Weather fluctuations, gender affect household's energy consumption-PIDS study. 2019. https://pids.gov.ph/ press-releases/325

30. Power Grid operator flags spike in demand. 2019. https:// www.bworldonline.com/power-grid-operator-flags-spikein-demand/

31. Energy in the Philippines. 2018. https://en.wikipedia.org/ wiki/Energy_in_the_Philippines

32. Energy Development Corporation: Good is why we've taken the lead. 2018. https://www.energy.com.ph/wp-content/uploads/2014/05/EDC-2013-Performance-Report.pdf

33. Power supply and demand highlights. 2017. https://www. doe.gov.ph/electric-power/2017-power-supply-and-demand-highlights-january-december-2017

34. Legal Information Archive: In The Matter of the Application for Approval of the Power Sales Agreement (PSA) between Zamboanga Del Sur I Electric Cooperative, Inc. (Zamsureco I) and Sarangani Energy Corporation (SEC), with prayer for provisional authority. 2018. http://lia.erc.gov.ph/documents/1682
35. Legal information archive : In the matter of the application for approval of the supplement to the Energy Supply Agreement (ESA) between Zamboanga Del Sur Ii Electric Cooperative, Inc. (Zamsureco II) and Therma Marine, Incorporated (TMI), with motion for Provisional Authority and Motion for Confidential Treatment of Information. 2015. http://lia.erc.gov.ph/documents/1742

36. Socoteco explores addt'l $30 \mathrm{MW}$ to help shorten brownouts. 2013. https://www.mindanews.com/top-stories/2013/03/ socoteco-explores-addtl-30-mw-to-help-shorten-brownouts/

37. Press Reader. 2018. https://www.pressreader.com/

38. Legal Information Archive: Legal Information Archive: In the matter of the application for approval of the Power Sales Agreement (PSA) between Davao Del Sur Electric Cooperative, Inc. and Hedcor Tudaya, Inc., with prayer for provisional authority. 2018. http://lia.erc.gov.ph/documents $/ 1340$

39. Power outages in GenSan, vicinity extend to 7 hours. 2013. https://www.mindanews.com/top-stories/2013/10/power-outages-in-gensan-vicinity-extend-to-7-hours/

40. Power Rate Increase. 2016. http://www.sukelco.com.ph/ index.php/2016/03/14/power-rate-increase/ 